\title{
Review \\ The Effects of Exercise and Activity-Based Physical Therapy on Bone after Spinal Cord Injury
}

\author{
Tommy W. Sutor $\left.{ }^{1,2}{ }^{(}\right)$, Jayachandra Kura ${ }^{1}$, Alex J. Mattingly ${ }^{3}$, Dana M. Otzel ${ }^{2}$ and Joshua F. Yarrow ${ }^{1,2,4, *(D)}$ \\ 1 Research Service, Malcom Randall Department of Veterans Affairs Medical Center, North Florida/South \\ Georgia Veterans Health System, Gainesville, FL 32608, USA; Thomas.Sutor@va.gov (T.W.S.); \\ jayachankura@ufl.edu (J.K.) \\ 2 Brain Rehabilitation Research Center, Malcom Randall Department of Veterans Affairs Medical Center, \\ North Florida/South Georgia Veterans Health System, Gainesville, FL 32608, USA; dotzel@ufl.edu \\ 3 Geriatrics Research, Education, and Clinical Center, North Florida/South Georgia Veterans Health System, \\ Gainesville, FL 32608, USA; mattingly@ufl.edu \\ 4 Division of Endocrinology, Diabetes, and Metabolism, University of Florida College of Medicine, \\ Gainesville, FL 32611, USA \\ * Correspondence: Joshua.Yarrow@medicine.ufl.edu; Tel.: +1-352-376-1611 (ext. 10-5234)
}

Citation: Sutor, T.W.; Kura, J.; Mattingly, A.J.; Otzel, D.M.; Yarrow, J.F. The Effects of Exercise and Activity-Based Physical Therapy on Bone after Spinal Cord Injury. Int. J. Mol. Sci. 2022, 23, 608. https:// doi.org/10.3390/ijms23020608

Academic Editor: Sebastien Couillard-Despres

Received: 3 November 2021 Accepted: 21 December 2021 Published: 6 January 2022

Publisher's Note: MDPI stays neutral with regard to jurisdictional claims in published maps and institutional affiliations.

Copyright: (C) 2022 by the authors. Licensee MDPI, Basel, Switzerland. This article is an open access article distributed under the terms and conditions of the Creative Commons Attribution (CC BY) license (https:// creativecommons.org/licenses/by/ $4.0 /)$.

\begin{abstract}
Spinal cord injury (SCI) produces paralysis and a unique form of neurogenic disuse osteoporosis that dramatically increases fracture risk at the distal femur and proximal tibia. This bone loss is driven by heightened bone resorption and near-absent bone formation during the acute postSCI recovery phase and by a more traditional high-turnover osteopenia that emerges more chronically, which is likely influenced by the continual neural impairment and musculoskeletal unloading. These observations have stimulated interest in specialized exercise or activity-based physical therapy (ABPT) modalities (e.g., neuromuscular or functional electrical stimulation cycling, rowing, or resistance training, as well as other standing, walking, or partial weight-bearing interventions) that reload the paralyzed limbs and promote muscle recovery and use-dependent neuroplasticity. However, only sparse and relatively inconsistent evidence supports the ability of these physical rehabilitation regimens to influence bone metabolism or to increase bone mineral density (BMD) at the most fracture-prone sites in persons with severe SCI. This review discusses the pathophysiology and cellular/molecular mechanisms that influence bone loss after SCI, describes studies evaluating bone turnover and BMD responses to ABPTs during acute versus chronic SCI, identifies factors that may impact the bone responses to $\mathrm{ABPT}$, and provides recommendations to optimize ABPTs for bone recovery.
\end{abstract}

Keywords: neuromuscular electrical stimulation; bodyweight supported treadmill training; vibration; osteoblast; osteoclast; osteocyte; sclerostin; Wnt beta catenin; RANKL; OPG

\section{Introduction}

An estimated 250,000 to 500,000 new spinal cord injuries (SCI) occur worldwide each year [1], with males representing $~ 80 \%$ of the population [2]. Roughly one-third of these are motor-complete SCIs that result in permanent sublesional paralysis, while the remainder are incomplete and retain voluntary contractility in some muscles that are innervated below the lesion [2]. Locomotor dysfunction is the most recognizable symptom of SCI and is accompanied by other medical consequences that develop in this population [3], including severe osteoporosis and high fracture risk [4], which worsen with increasing SCI severity [5-9] and injury duration [10,11].

Bone loss after SCI is termed neurogenic or disuse osteoporosis and is confined to the sublesional skeleton [10-12], with the most rapid and prevalent bone deficits occurring at the distal femur and proximal tibia regions [11,13,14]. At these sites, 50-100\% lower trabecular bone mineral density (BMD) develops in individuals within the first two to 
three years of SCI $[11,13,14]$, and $40-80 \%$ lower cortical bone mass exists several years after injury [14]. Some evidence also indicates that cortical bone becomes more porous after SCI [15]. Collectively, these bone deficits imply a significant weakening of skeletal integrity which likely influences the 20- to 100-fold higher fracture risk in persons with SCI when compared with the general population [16].

After SCI, fractures are usually non-traumatic, due to the mobility limitations in persons with SCI, and result from low-velocity compressive forces or torsional stresses [17] that develop while seated or during transfers to, or falls from, a wheelchair. These fractures most commonly occur at the epiphysis or metaphysis [5] of the distal femur [18] or proximal tibia $[7,8]$, where bone loss is the most severe and may require extended inpatient hospitalization [5]. Moreover, a single fracture more than doubles the risk for other secondary medical comorbidities after SCI, including venous thromboembolic events, respiratory illnesses, and pressure ulcers, among others [19]. These comorbidities influence the $30 \%$ higher five-year mortality risk for those of any age who fracture after SCI and the more than three-fold higher five-year mortality risk for persons with SCI who fracture after age 50 years [20]. The severe bone loss, high fracture incidence, and the associated morbidity and mortality indicate the need to improve osteoporosis screening and to develop evidence-based guidelines to prevent and treat osteoporosis in the SCI population [21,22].

\section{Determining BMD and Fracture Risk after SCI}

Dual-energy X-ray absorptiometry (DXA) is the standard to assess osteoporosis and fracture risk at traditional osteoporosis sites (e.g., lumbar spine and hip), via the quantification of areal (a)BMD and T-scores [23]. Specialized DXA techniques have also been developed to assess distal femur and/or proximal tibia aBMD after SCI [17,24,25], although not all the DXA systems are capable of imaging these locations and T-scores have not been established at these sites. As such, some have suggested using traditional osteoporosis sites as surrogates for the distal femur and proximal tibia. However, BMD changes occur more rapidly at the knee than at other bone sites after SCI [26]. Furthermore, after SCI, aBMD changes at the knee are only moderately correlated with the total hip and femoral neck aBMD and T-scores [26,27] and may not correspond with the degree of bone loss at the hip or femoral neck [28-31], with significant predictive inaccuracy between the sites that surround the knee and hip [27]. Alternatively, peripheral quantitative computerized tomography (pQCT) yields volumetric (v)BMD of the trabecular and cortical bone compartments and has been used to estimate vBMD fracture thresholds at the distal femur epiphysis $\left(<114 \mathrm{mg} / \mathrm{cm}^{3}\right)$ and distal tibia epiphysis $\left(<71 \mathrm{mg} / \mathrm{cm}^{3}\right)$ in persons with SCI [32] High-resolution (HR)-pQCT with finite element analysis (FEA) [33] can also measure vBMD, along with the bone microstructural parameters, and can simultaneously model bone tensile properties, providing insight into bone microarchitecture and mechanical alterations that contribute to increased fracture risk after SCI [34,35]. For example, the reduction in proximal femur bone strength that was estimated via FEA was three times greater than the aBMD loss that was determined by DXA over the first few months after SCI [36], likely because DXA cannot discern trabecular vs. cortical BMD nor quantify other bone parameters that influence fracture risk [37]. Regardless, the limited availability of pQCT systems restricts their practicality and highlights the continued need for DXA evaluations to identify fracture risk in persons with SCI.

\section{Pathophysiology of SCI-Induced Bone Loss}

Within the SCI population, persons with complete paralysis display the most extensive bone loss $[9,26]$ and highest fracture risk [8], likely because the residual voluntary muscle function lessens bone loss. As evidence, persons with incomplete SCI display less bone loss in the lesser impaired limb [38]. Moreover, cast immobilization (a technique that limits muscle contraction and restricts voluntary joint motions) has been shown to worsen bone loss in a rat severe SCI model [39], demonstrating that even a minimal degree of residual muscle contractility that accompanies severe SCI assists in preserving BMD. These 
observations support the understanding that disuse is a factor mediating SCI-induced bone loss. However, bone loss after SCI is distinct from that which is occurring in response to other disuse conditions in both severity and mechanism. For example, in humans with complete SCI, trabecular and cortical bone loss occurs at a rate nearing $1 \%$ per week over the initial few months post-SCI [40], which is 4-10 times faster than in other types of disuse (e.g., prolonged bed rest or microgravity exposure) [41]. Similarly, in rodent SCI models, bone loss is two or more times faster than that resulting from cast immobilization [39] or sciatic neurectomy [42]. These findings suggest that other factors that are occurring secondary to SCI may worsen bone loss, including systemic hormonal changes, altered bone innervation [43], and/or impaired bone perfusion [44]. For further discussion, readers are directed to the following review [4].

\section{Bone Turnover after SCI}

Bone undergoes continuous remodeling throughout the lifespan, which is balanced during homeostasis via integrated resorption and formation processes that maintain skeletal integrity. However, after severe SCI, a unique form of unopposed bone resorption drives bone loss [4]. As evidence, Minaire et al. examined persons with SCI and observed signs of increased osteoclastic resorption that accompanied a near-absence of surface-level bone formation at the iliac crest [45] that is suggestive of uncoupled bone turnover. Circulating bone resorption markers are also several-fold higher than the upper reference ranges in persons with SCI throughout the acute (<four-months) to subacute (4-12 months) postinjury periods when bone loss is most rapid, while circulating bone formation markers remain near reference ranges [10,46-51]. Similarly, in rodent models of severe SCI, the use of dynamic histomorphometry has revealed that trabecular bone resorption persists at the distal femur and proximal tibia in the near absence of bone formation throughout the initial one to three-weeks post-SCI [44,52-57], when nearly all the trabecular bone loss occurs. Thereafter, trabecular bone formation renormalizes [58,59] and bone loss slows [52].

\section{Mechanisms Regulating Bone Loss after SCI}

The molecular mechanisms that propagate the uncoupled bone turnover that is present in the paralyzed limbs after SCI and that drive the exceedingly rapid bone loss in this population, in comparison with other disuse conditions, require further elucidation. Given that disuse is a factor that mediates SCI-induced bone loss, it is likely that osteocytes (primary bone mechanosensor) influence neurogenic osteoporosis. Osteocytes reside within the calcified bone matrix and communicate with other osteocytes and with osteoclasts, osteoblasts, and other cells that reside on bone surfaces via dendritic projections that emerge from the osteocyte cell body to form an interconnected dendritic network. Dendrites provide one means by which the osteocytes sense alterations in localized bone strains that result from disuse or imposed loading and transduce this information to the osteocyte cell body, a process that is referred to as mechanotransduction. In response to this stimulus, osteocytes release a host of nuclear-derived signaling molecules (e.g., receptor activator of NF- $\mathrm{kB}$ ligand (RANKL), osteoprotegrin (OPG), sclerostin, and others) that orchestrate osteoclastic and/or osteoblastic bone remodeling. Readers are directed to the following reviews that discuss osteocyte mechanosensors and mechanotransduction-associated signaling pathways $[60,61]$.

While it is likely that osteocytes orchestrate the skeletal responses to SCI, few studies have directly assessed this possibility or described how osteocytes respond to paralysis or to imposed bone loading after SCI. Qin et al. observed osteocyte morphological aberrations in rats within seven-weeks of spinal transition, including reductions in the dendritic length and dendritic number, along with altered osteocyte cell body shape [62]. The molecular mechanisms that regulate these morphologic changes require further elucidation. However, administration of a monoclonal sclerostin antibody that binds and inactivates the circulating sclerostin was shown to preserve the dendritic length and osteocyte cell body morphology in this model [62], suggesting potential autocrine regulation. Regardless, 
it remains unknown whether the osteocyte morphological aberrations that are occurring in the paralyzed limbs produce functional consequences (e.g., impaired ability to detect imposed bone strains) within the dendritic network.

\section{RANKL Signaling}

RANKL is an osteocyte-derived protein that is necessary for the differentiation of hematopoietic progenitors of the monocyte-macrophage lineage into osteoclasts. RANKL stimulates osteoclastogenesis and bone resorption by binding RANK receptors on the cell surfaces of osteoclast precursors and osteoclasts, respectively [63]. RANKL-mediated osteoclastic resorption is primarily modified in response to secreted concentrations of RANKL and OPG, an endogenous decoy receptor for RANKL that is produced by osteoblast-lineage cells and prevents RANK binding. The relative abundance of RANKL to OPG is a key factor that influences RANKL signaling, with higher RANKL and/or lower OPG stimulating bone resorption and osteoclastogenesis. Readers are directed to the following review for an overview of RANKL signaling in bone biology [63].

Signs of altered RANKL signaling coexist with bone loss in rodent SCI models. For example, cultured bone marrow mesenchymal or stromal cells that were isolated from spinalized mice exhibit higher RANKL and lower OPG vs. the controls, which may underlie the two- to three-fold increase in tartrate-resistant acid phosphatase (TRAP)+ osteoclastlike cells that develop in bone marrow culture that are derived from spinalized mice [64]. Similarly, in rodent SCI models, RANKL mRNA and protein expressions were 75-300\% higher and OPG mRNA and protein were 30-75\% lower at the distal femur and proximal tibia when compared with the controls. Recently, Cirnigliaro et al. [65] reported that 12-mo treatment with Denosumab, a human monoclonal antibody that binds and antagonizes RANKL activity, completely prevented distal femur and proximal tibia aBMD loss in a small cohort of persons with acute SCI. Gifre et al. reported that Denosumab increased total hip, femoral neck, and lumbar spine aBMD in persons with subacute to chronic SCI [66], with BMD gains being associated with the magnitude of RANKL suppression [67]. Collectively, these data indicate that rodent SCI models display an altered RANKL:OPG ratio in a manner that would be expected to promote bone loss, and that pharmacologic RANKL inhibition prevents aBMD loss at the most fracture prone sites after SCI and increases $\mathrm{aBMD}$ at other sites.

Recently, RANK [68] and RANKL [69] have also been identified as functional components of extracellular vesicles (EVs) that are secreted directly from osteoclasts and osteoblasts, respectively, providing a mechanism for intercellular communication [70]. In this regard, RANK in osteoclast-derived EVs can bind osteoblastic RANKL and stimulate a reverse RANKL signaling pathway that promotes bone formation and that couples bone resorption and formation processes [71]. Osteoblast-derived RANKL-containing EVs can also promote osteoclastogenesis in vivo, at least when delivered to transgenic RANKL deficient mice [72]. Given these observations, it is enticing to imagine that the uncoupled bone turnover that is present in the paralyzed limbs after SCI may be influenced by transient alterations in RANK or RANKL containing EVs, although, this remains to be determined.

\section{Canonical Wnt/ $\beta$-Catenin Signaling and Sclerostin}

The canonical Wnt/ $\beta$-catenin signaling cascade promotes bone formation by stimulating osteoblast differentiation and osteoblast growth rate, by inhibiting osteoblast apoptosis, and by stimulating osteoblast activity. This signaling pathway is initiated when the various Wnt ligands (e.g., Wnt1 and Wnt3a) bind the low-density lipoprotein receptor-related protein (LRP)5/6 and Frizzled receptor complex on osteoblasts and prevent the Axinadenomatous polyposis coli-glycogen synthase kinase 3-casein kinase 1 (APC-GSK-3-Ck1) complex from phosphorylating cytoplasmic $\beta$-catenin, which traditionally marks $\beta$-catenin for proteasomal degradation. In response, unphosphorylated $\beta$-catenin accumulates within the cytoplasm, where it can be shuttled to the nucleus to interact with DNA binding proteins (e.g., TCF/LEF) to promote the activation of Wnt responsive gene transcription 
pathways [61]. Several negative regulators of Wnt-signaling exist, including sclerostin, which binds the LRP5/ 6 receptors and prevents Wnt ligands from initiating Wnt-signaling. Sclerostin is known to mediate bone loss that develops in response to unloading [73] and bone gain resulting from imposed skeletal strains, with sclerostin suppression being essential for the osteogenic responses to mechanical loading [74]. Readers are directed to the following review for further discussion on Wnt signaling in bone [61].

Alterations in Wnt signaling accompany bone loss in the paralyzed limbs in rodent SCI models. For example, several proteins that are involved in Wnt signaling (LRP5, Wnt1, and Wnt3) are repressed at the distal femur and proximal tibia within a few weeks of SCI, while the number of osteocytes that stain for sclerostin are increased [75] and SOST (gene encoding sclerostin) is $300 \%$ higher. These changes likely influence the $50 \%$ lower $\beta$-catenin protein expression that has been observed at the proximal tibia after $\mathrm{SCI}$, along with the reduced $\beta$-catenin expression that is present in cultured mesenchymal cells that are derived from spinal transected mice. Elevated sclerostin also appears to mediate SCI-induced bone loss, given that SOST-/- mice are protected against bone loss and suppressed osteoblastogenesis after spinal transition [76]. Moreover, a pharmacologic sclerostin antibody that binds and inactivates sclerostin has been shown to completely prevent distal femur and proximal tibia bone loss in rodents when it is delivered immediately after severe incomplete [53] to complete SCI [62] by promoting bone formation, and to reverse trabecular and cortical bone loss when it is delivered after bone loss emerges [77].

\section{Activity-Based Physical Therapy (ABPT) after SCI}

Given the role of disuse in mediating the musculoskeletal decline after $\mathrm{SCI}$, a large body of research has focused on ABPTs and other exercise-based regimens that reload the impaired limbs to restore muscle integrity [78]. Common ABPTs include overground locomotor training and/or bodyweight-supported treadmill training (BWSTT) that is accompanied by manual or robotic-assisted placement of the impaired limbs into normal gait patterns, passive cycling, or functional electrical stimulation (FES) that is coupled with cycling, rowing, or resistance training (RT). With intense repetitive training, ABPTs are theorized to activate and optimize sublesional spinal networks, enabling the improved performance of task-specific motor activities [79]. For example, BWSTT and other locomotor-based modalities have shown promise in promoting use-dependent neuroplasticity after mild to moderate motor-incomplete SCI and result in improved walking speed, temporal gait parameters, and lower limb muscle activation patterns [80-82], along with increased muscle strength and rate of torque development in some persons with incomplete SCI [78]. Structural and functional plasticity likely drives motor recovery resulting from these ABPTs and stems from the reorganization of both supraspinal and spinal cord neural circuits [83,84]. As evidence, beneficial adaptations to spinal neuronal pathways have been observed in response to ABPT by probing soleus Hoffmann reflex during walking in which homosynaptic facilitation normalized, homosynaptic depression reversed, and presynaptic inhibition of Ia afferents improved $[83,85,86]$. The functional recovery in persons with incomplete SCI undergoing ABPT has also been associated with findings that indicate greater descending corticospinal drive, including increased ankle dorsiflexor and knee extensor maximal motor-evoked potentials, a probe of corticospinal tract excitability [87], and improved ankle dorsiflexor and plantar flexor muscle co-activation patterns during walking [88]. Possible mechanisms underlying ABPT-mediated neuroplasticity may involve the upregulation of brain-derived neurotrophic factor and/or its receptor, tyrosine kinase B mRNA, in the spinal cord, which mediate improvements of synaptic transmission, axon regeneration, and motor neuron survival [89]. For further discussion on this topic, we refer readers to the following review [78].

\section{Effects of ABPT and Reloading Modalities on Bone after SCI}

In uninjured persons, weight-bearing exercise that produces high peak strains and/or high strain rates increases BMD [90] and prevents disuse-mediated bone loss [91]. In vari- 
ous models, cyclical loading has also been shown to stimulate bone formation and increase bone mass in a manner that is dependent upon the compressive strain that is applied to bone, the loading frequency $(\mathrm{Hz})$, and the loading duration. In contrast, stationary/static loading does not typically alter bone parameters [92]. This knowledge has contributed to an emphasis on ABPTs to increase BMD after SCI [17] and to recommendations from healthcare providers to utilize weight-bearing activities to improve BMD after SCI [93]. However, evidence demonstrating the skeletal benefits of ABPTs is sparse in persons with SCI with several meta-analyses concluding that insufficient evidence exists to establish their effectiveness in improving BMD [94,95]. Likewise, the existing evidence is contradictory when assessing the ability of ABPTs to alter bone turnover in a manner that would be expected to improve BMD after SCI. For example, Bloomfield et al. [96] reported that nine-months of FES cycling increased serum osteocalcin (bone formation marker) $>75 \%$ in persons with chronic complete SCI. Similarly, Mobarke et al. [97] reported that 12-weeks of BWSTT starting at $50 \%$ bodyweight support and progressing to full weight-bearing increased osteocalcin when compared with stretching and RT exercises, while others have reported that sixto eight-months of BWSTT suppressed urinary deoxypyridinoline/creatinine (bone resorption marker) without altering osteocalcin [50]. In comparison, Craven et al. [98] reported that those with chronic incomplete SCI displayed no change in circulating osteocalcin, CTX (bone resorption marker), or sclerostin in response to four-months of FES assisted BWSTT. Similarly, others reported that persons with incomplete to complete SCI exhibit little change in circulating bone formation markers or circulating/urinary bone resorption markers in response to FES-based cycling [30] or RT [51,99], BWSTT [100], seated [101] or standing vibration [102], or a multimodal ABPT regimen [103].

Relatively few preclinical SCI studies have evaluated the possibility that ABPTs alter bone turnover or improve BMD, likely because few preclinical modalities exist to reload the paralyzed limbs. In this regard, Qin et al. developed an implantable electrical stimulation (ES) system that elicited unilateral near-maximal contractions of the paralyzed soleus and plantaris muscles in spinal transected rats, with ES delivered $60 \mathrm{~min} /$ day for one-week (40 Hz at $1.5 \mathrm{~V}$, (2 s on/18 s off), $1.5 \mathrm{~V}$ ) [64]. This brief ES protocol improved plantaris muscle mass but did not alter BMD or bone microstructure when compared with SCI rats not receiving ES. However, ES suppressed circulating CTX by $\sim 50 \%$ and reduced the number of osteoclast-like TRAP-positive osteoclasts that develop in ex vivo cultures, suggesting ES suppressed osteoclastogenesis and bone resorption. ES also reversed the suppression of OPG mRNA in ex vivo osteoblast cultures, without altering RANKL mRNA, and suppressed mRNA expression of SOST, along with several other genes that negatively regulate Wnt-signaling. Despite these changes, ES did not reverse the SCI-induced suppression of osteocalcin nor did it alter the reduction in alkaline phosphatase-positive or von Kossastained colonies that develop in ex vivo osteoblast cultures that are derived from tibia or femur bone marrow, suggesting that one-week of ES did not stimulate osteoblastogenesis or bone formation. More recently, the same group reported that a four-week ES protocol lessened distal femur and proximal tibia aBMD loss and increased several trabecular and cortical bone microstructural variables at the distal femur and femoral midshaft [104]. This four-week ES protocol also increased the circulating osteocalcin, suggesting an increased whole-body bone formation. However, distal femur mineralizing surface (index of active bone formation), mineral apposition rate (index of osteoblast activity), and surface-level bone formation were similarly suppressed in SCI+ES and SCI groups vs. the controls, providing no direct evidence that ES stimulated bone formation at this site. Furthermore, ES did not prevent the suppression of osteoblastogenesis that resulted from SCI, nor did it increase osteoblast SOST mRNA expression. However, ES reduced the RANKL:OPG mRNA ratio within the hindlimbs, reduced the number of TRAP+ osteoclasts in ex vivo cultures, and produced a 50\% reduction in the percentage of trabecular bone that was covered by osteoclasts when compared with the untreated SCI animals, providing evidence of an antiresorptive effect. 
Beyond the studies that are described above, we are aware of only a few others assessing bone responses to loading in preclinical SCI models. Zamarioli et al. [105] evaluated two loading protocols in spinal transected rats: (1) ES of the quadriceps and triceps surae muscles [ $300 \mu$ s pulses, $50 \mathrm{~Hz}$ ( $5 \mathrm{~s}$ on/15 s off), 20-150 mA, 3 days/week, $30 \mathrm{~min} /$ day] and (2) a custom-built bipedal standing frame that resulted in $70 \%$ of total body mass being supported by the hindlimbs. Neither modality prevented distal femur or proximal tibia aBMD loss nor lessened the reduction in bone strength at these sites, although, the maximal strength of the lumbar vertebrae increased by $\sim 30 \%$ in response to bipedal standing. We have also reported that hindlimb cast immobilization worsened trabecular and cortical bone loss at the distal femur and proximal tibia and reduced the distal femur bone strength in a severe SCI model [39]. In this study, two-weeks of quadrupedal (q)BWSTT reversed the bone loss that resulted from cast immobilization but did not lessen bone loss that resulted directly from SCI. In a follow-up study, three-weeks of qBWSTT was also shown not to attenuate trabecular bone deterioration after SCI [59].

Based on the evidence that is presented above, it remains unknown whether ABPTs that reload the impaired limbs after SCI alter bone turnover in a manner that is sufficient to improve BMD. With the above considered, the next sections summarize the findings of clinical studies that met the following criteria: (1) enrolled adults with incomplete and/or complete SCI and (2) reported BMD at the distal femur or proximal tibia, or BMD or T-scores at other sublesional sites, both before and after an ABPT intervention regardless of other study characteristics. In doing so, we considered several important questions. First, did the study enroll persons with acute to subacute (0-12-months) SCI when bone loss is the most rapid, or during the chronic ( $>12$-month) period when bone loss is more gradual? Secondly, did the study assess the bone changes at the relevant fracture-prone sites after SCI (i.e., distal femur or proximal tibia) or at other bone sites? Thirdly, did the study report that an ABPT attenuated bone loss or increased BMD? We considered trials that reported attenuated bone loss or increased BMD vs. the baseline or a control group to have demonstrated improvement, while those not meeting these criteria were considered to have shown no effect. For studies that combined an ABPT with a pharmacologic intervention, we only discuss the groups that were receiving ABPT alone. Case studies, case series, and cross-sectional studies that did not statistically assess the pre-post differences are discussed from a qualitative perspective.

\section{ABPT Interventions and BMD after Acute/Subacute SCI}

A visual summary of the studies examining the effects of different ABPT modalities at knee and non-knee sites in acute and subacute SCI is presented in Figure 1A,B. Case studies or case series that enrolled persons in the acute to subacute post-SCI period utilized BWSTT with manual- [50] or robotic-assistance [38], or FES rowing [106]. Of these, none reported increased BMD vs. baseline. Only FES rowing appeared to attenuate the distal femur BMD loss when compared with the expected bone loss rate during the subacute SCI phase [106], while neither BWSTT with manual- [50] or robotic-assistance [38] produced an apparent effect on BMD (Table 1). Using a cross-sectional design, Goemaere et al. [107] also reported that those with incomplete or complete SCI who initiated passive standing during the acute SCI phase displayed less aBMD deficits at the lumbar spine [0\% difference (standing) vs. $-7.4 \%$ (non-standing)] and femoral shaft [ $-21 \%$ (standing) vs. $-29.2 \%$ (non-standing)] when compared with normative aBMD values that were derived from a non-SCI cohort, although, standing did not appear to attenuate aBMD deficits at the total hip or femoral neck or trochanter. 
Table 1. Case studies, case series, and cross-sectional studies evaluating the effects of activity-based physical therapy (ABPT) and/or loading on bone mineral density (BMD) in adults with acute or subacute spinal cord injury (SCI).

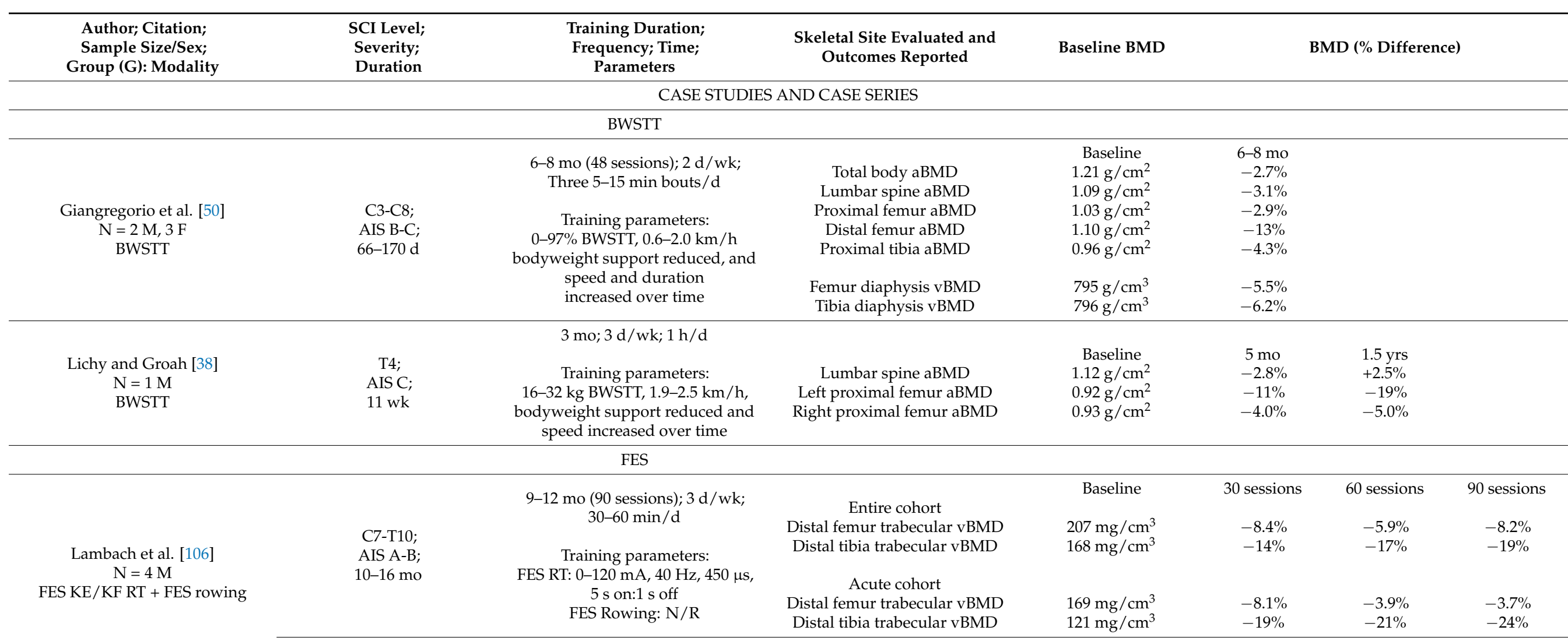

Note: Lambach et al. included N $=2<1$ yr and $\mathrm{N}=2$ chronic SCI. Baseline and \% difference BMD values are reported separately for the entire cohort and the subacute SCI cohort. Values for the chronic cohort are in Table 3 
Table 1. Cont.

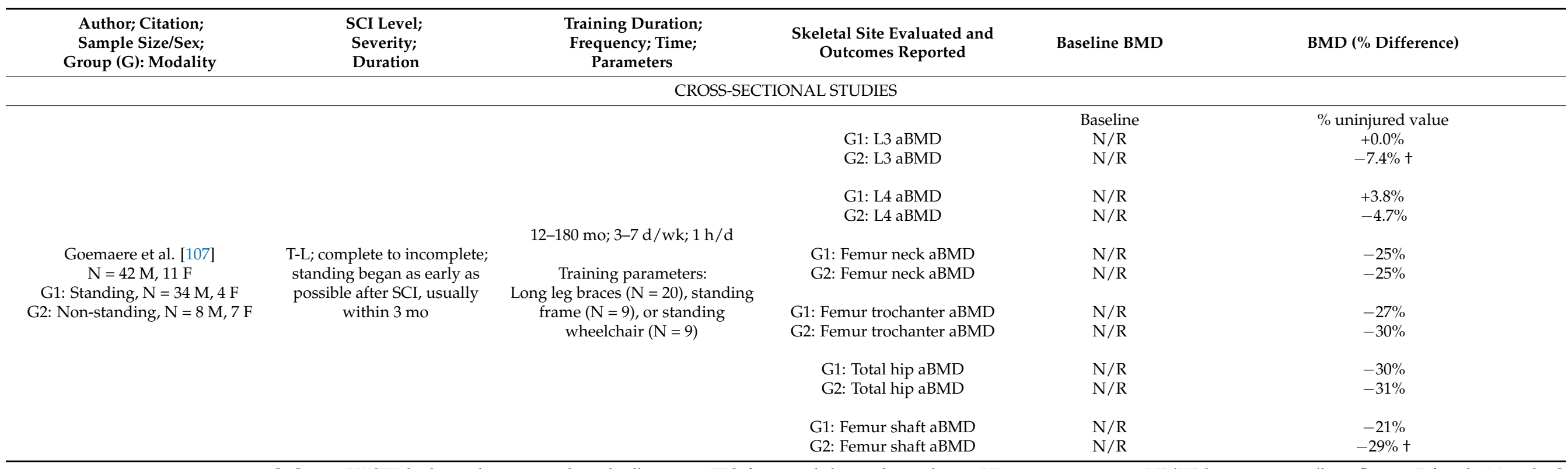

G, Group; BWSTT, bodyweight-supported treadmill training; FES, functional electrical stimulation; RT, resistance training; KE/KF, knee extension/knee flexion; F, female; M, male; cervical; T, thoracic; AIS, American Spinal Injury Association Impairment Scale; SCI Duration: time since SCI in relation to start of intervention; aBMD, areal bone mineral density; vBMD, volumetric bone mineral density; min, minute; h, hour; d, day; wk, week; mo, month; N/R, not reported. The \% change was reported in individual papers or was manually calculated from the data in tables and/or figures; + indicates a $p$ value of $<0.05$ between the groups; a lack of symbols indicates no statistical differences that were reported versus the baseline or between the groups. 


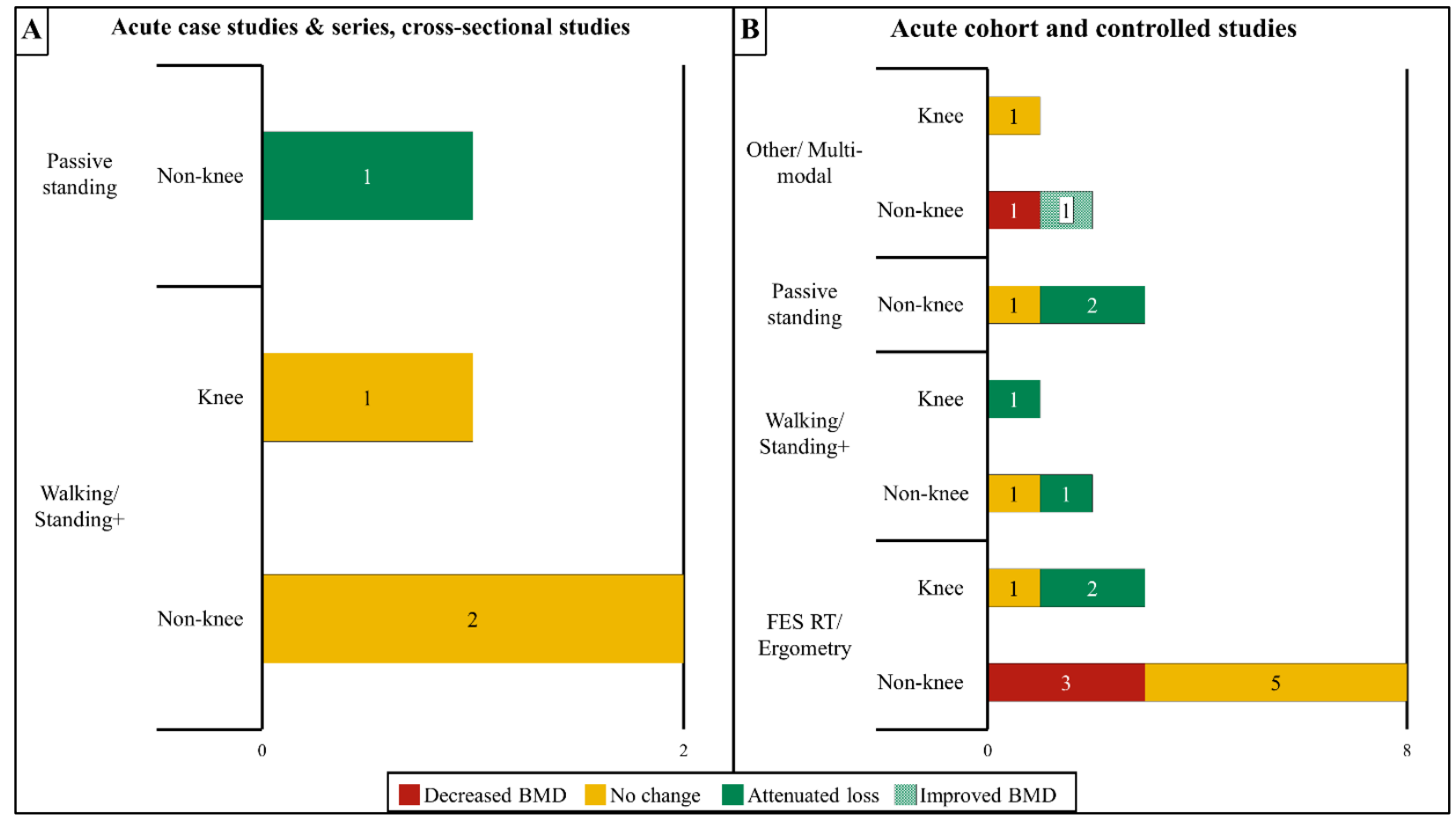

Figure 1. Summary of the effects of activity-based physical therapy (ABPT) on changes in bone mineral density (BMD) at the knee (distal femur and proximal tibia) or all other sublesional non-knee sites in persons with acute/sub-acute SCI for (A) case studies, case series, and cross-sectional studies or (B) controlled and cohort studies. The total number of studies that reported decreased BMD, no BMD change, attenuated BMD loss, or improved BMD after an ABPT modality are provided in the corresponding bars. Some studies examined both knee and non-knee sites - in these instances, the individual results for knee and non-knee sites were included in each bar. If a study compared more than one modality, the individual results for each modality were included if available. If no studies were found that fit a certain category, the category was omitted from the chart. Overall, most acute studies showed no effect or attenuated BMD loss over time. FES, functional electric stimulation; RT, resistance training; "Standing+" refers to interventions that combined standing modalities with other modalities that increase muscle activation such as FES or vibration.

No trial without a control comparator [103,108] nor any controlled trial [28,31,51,99,109-115] that enrolled persons in the acute/subacute period reported increased sublesional BMD vs. the baseline, irrespective of the site that was evaluated (Table 2). There were two uncontrolled trials that reported no obvious attenuation of sublesional bone loss after ABPT. Rodgers et al. [108] reported that distal tibia trabecular vBMD was 5.4\% lower vs. the baseline in a cohort of persons with subacute or chronic SCI involved in a 12-week FES RT protocol, with the subacute SCI participant displaying the greatest bone loss ( $-26 \%$ vs. the baseline) among all the participants. Astorino et al. [103] also reported that a cohort of persons with acute/subacute SCI or chronic SCI exhibited progressively lower aBMD at most sites that were examined, including the distal femur and proximal tibia, while undergoing a six-month multimodal ABPT regimen that involved lower extremity RT, BWSTT, overground walking, vibration training, and FES cycling. Similarly, 5 of the 11 controlled trials did not detect attenuated sublesional BMD loss at any skeletal site that was examined [51,99,110,111,114]. These trials ranged from 6-weeks to 12-months in duration (training frequency three to five days/week) and used the following modalities: passive unilateral standing [110], BWSTT with standing [114], and FES-based RT [51,99,111]. Of these, only Groah et al. [51] examined BMD at the knee. 
Table 2. Uncontrolled and controlled interventional studies evaluating the effects of activity-based physical therapy (ABPT) and/or loading on bone mineral density (BMD) in adults with acute or subacute spinal cord injury (SCI).

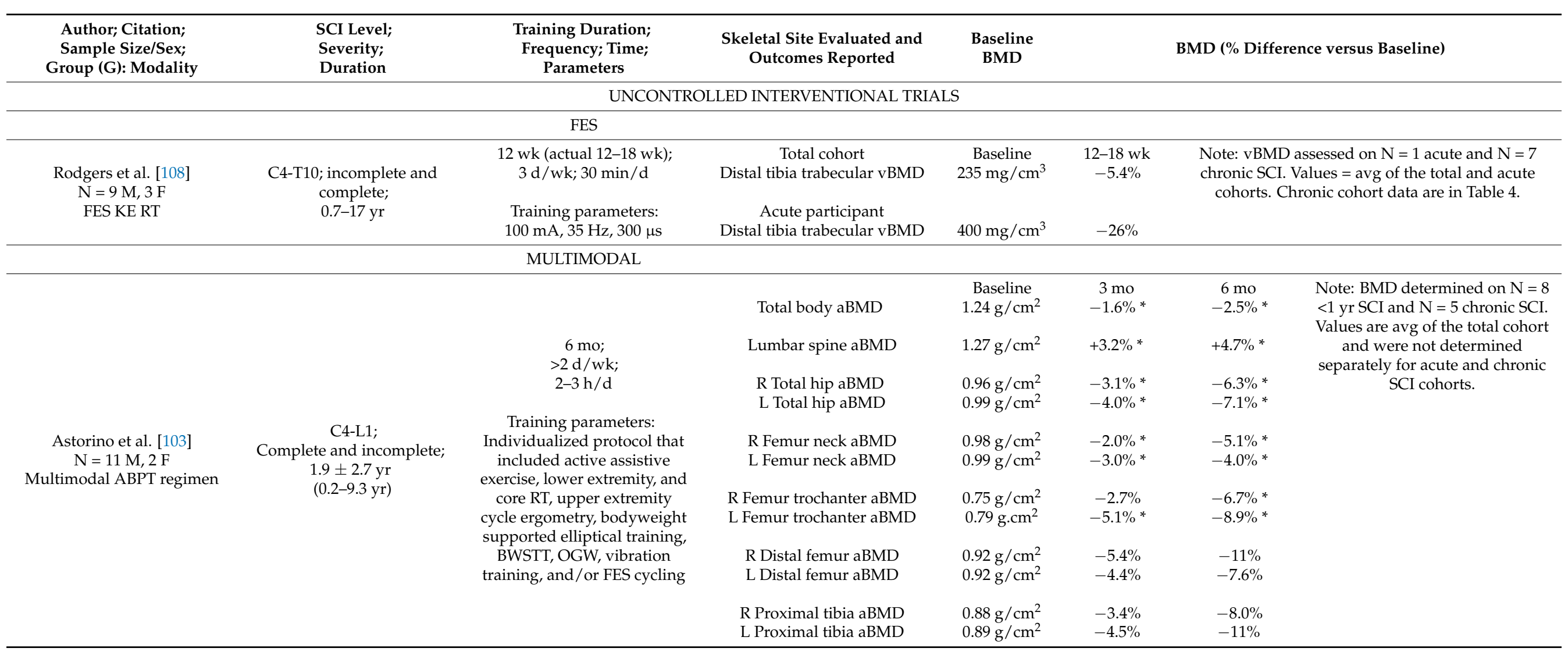


Table 2. Cont.

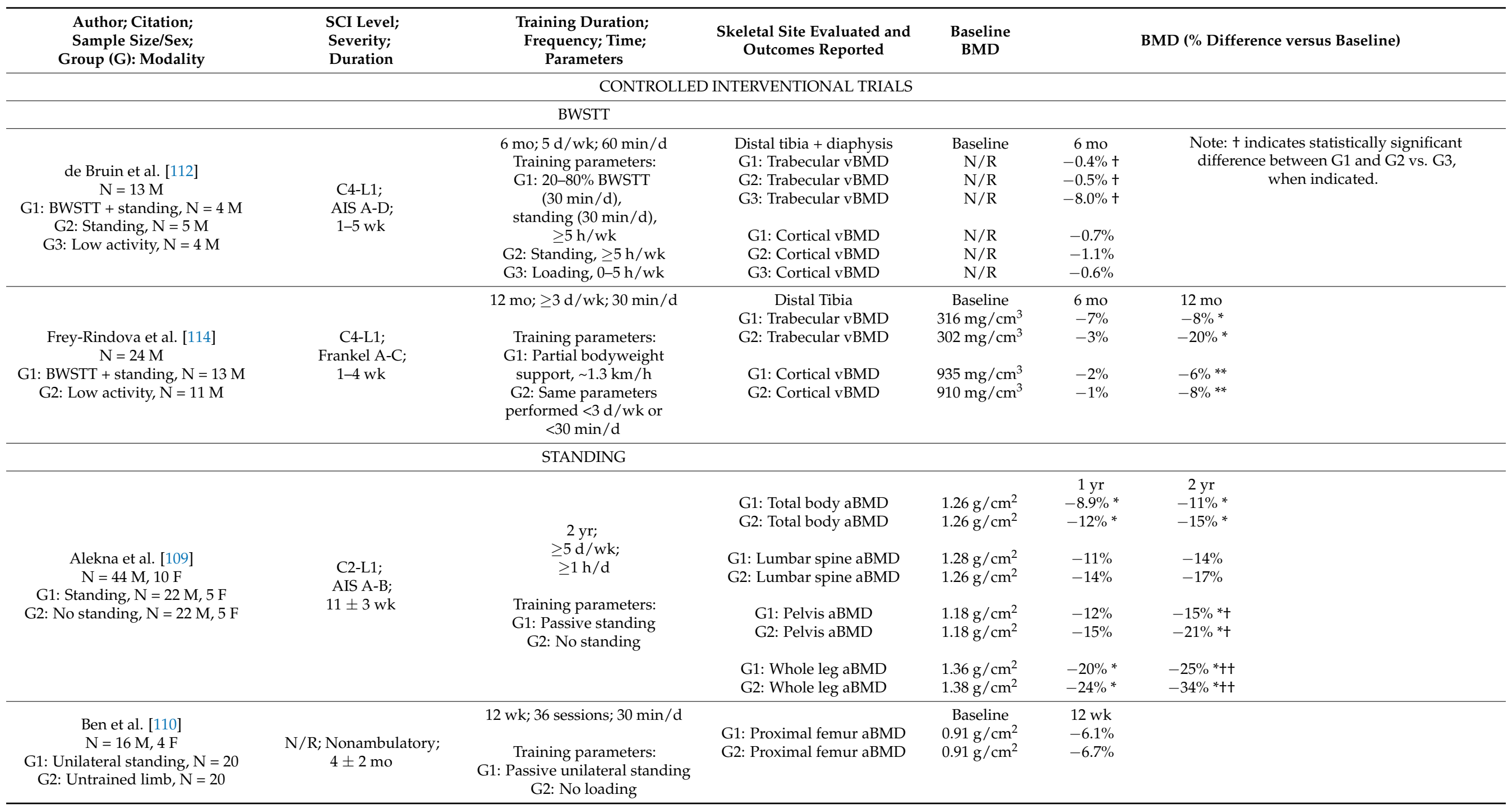


Table 2. Cont.

\begin{tabular}{|c|c|c|c|c|c|c|}
\hline $\begin{array}{c}\text { Author; Citation; } \\
\text { Sample Size/Sex; } \\
\text { Group (G): Modality }\end{array}$ & $\begin{array}{l}\text { SCI Level; } \\
\text { Severity; } \\
\text { Duration }\end{array}$ & $\begin{array}{l}\text { Training Duration; } \\
\text { Frequency; Time; } \\
\text { Parameters }\end{array}$ & $\begin{array}{l}\text { Skeletal Site Evaluated and } \\
\text { Outcomes Reported }\end{array}$ & $\begin{array}{l}\text { Baseline } \\
\text { BMD }\end{array}$ & & BMD (\% Difference versus Baseline) \\
\hline & & FES/NMES & & & & \\
\hline \multirow{16}{*}{$\begin{array}{l}\text { Arija-Blazquez et al. [99] } \\
\quad \mathrm{N}=8 \mathrm{M} \\
\text { G1: FES RT, } \mathrm{N}=5 \mathrm{M} \\
\text { G2: SHAM FES, } \mathrm{N}=3 \mathrm{M}\end{array}$} & \multirow{16}{*}{$\begin{array}{c}\text { T4-T12; } \\
\text { AIS A; } \\
\text { G1: } 5.5 \pm 1.1 \mathrm{wk} \\
\text { G2: } 5.8 \pm 1.7 \mathrm{wk}\end{array}$} & \multirow{16}{*}{$\begin{array}{c}\text { Training parameters: } \\
\text { Both: } 8 \text { sets } \times 10 \text { contractions } \\
\text { G1: } 0-140 \mathrm{~mA}, 30 \mathrm{~Hz}, 200 \mu \mathrm{s} \\
\text { G2: } 0 \mathrm{~mA}, 30 \mathrm{~Hz}, 200 \mu \mathrm{s}\end{array}$} & & Baseline & $14 \mathrm{wk}$ & \\
\hline & & & G1: Lumbar spine aBMD & $0.91 \mathrm{~g} / \mathrm{cm}^{2}$ & $+3.5 \%$ & \\
\hline & & & G2: Lumbar spine aBMD & $1.23 \mathrm{~g} / \mathrm{cm}^{2}$ & $+2.1 \%$ & \\
\hline & & & Femur & & & \\
\hline & & & G1: Total hip aBMD & $0.92 \mathrm{~g} / \mathrm{cm}^{2}$ & $-7.0 \%$ & \\
\hline & & & G1: Femur neck aBMD & $0.79 \mathrm{~g} / \mathrm{cm}^{2}$ & $-7.6 \%$ & \\
\hline & & & G2: Femur neck aBMD & $0.96 \mathrm{~g} / \mathrm{cm}^{2}$ & $-8.3 \%$ & \\
\hline & & & & & & \\
\hline & & & G1: Ward triangle aBMD & $0.65 \mathrm{~g} / \mathrm{cm}^{2}$ & $-6.5 \%$ & \\
\hline & & & G2: Ward triangle aBMD & $0.86 \mathrm{~g} / \mathrm{cm}^{2}$ & $-7.7 \%$ & \\
\hline & & & & & & \\
\hline & & & G1: Trochanter aBMD & $0.70 \mathrm{~g} / \mathrm{cm}^{2}$ & $-9.9 \%$ & \\
\hline & & & G2: Trochanter aBMD & $0.83 \mathrm{~g} / \mathrm{cm}^{2}$ & $-8.1 \%$ & \\
\hline & & & G1: Intertrochanteric aBMD & $1.05 \mathrm{~g} / \mathrm{cm}^{2}$ & $-5.9 \%$ & \\
\hline & & & G1: Whole leg aBMD & $1.19 \mathrm{~g} / \mathrm{cm}^{2}$ & $-2.9 \%$ & \\
\hline & & & G2: Whole leg aBMD & $1.44 \mathrm{~g} / \mathrm{cm}^{2}$ & $-3.3 \%$ & \\
\hline \multirow{10}{*}{$\begin{array}{c}\text { Clark et al. [29] } \\
\mathrm{N}=33(\mathrm{~N} \text { per sex } \mathrm{N} / \mathrm{R}) \\
\text { G1: FES RT, } \mathrm{N}=23 \\
\text { G2: Inactive } \mathrm{SCI} \text { control, } \mathrm{N}=10\end{array}$} & \multirow{10}{*}{$\begin{array}{l}\text { C4-T12; } \\
\text { AIS A-D; } \\
3 \mathrm{wk}\end{array}$} & & & Baseline & $3 \mathrm{mo}$ & $6 \mathrm{mo}$ \\
\hline & & & G1: Total body aBMD & $N / R$ & $-2.2 \%+\dagger$ & $-3.0 \%$ \\
\hline & & $\begin{array}{l}6 \mathrm{mo} \\
5 \mathrm{~d} / \mathrm{wk}\end{array}$ & G2: Total body aBMD & $\mathrm{N} / \mathrm{R}$ & $+0.7 \%++$ & $-1.9 \%$ \\
\hline & & $30 \mathrm{~min} / \mathrm{d}$ & G1: Lower extremity aBMD & $\mathrm{N} / \mathrm{R}$ & $-2.4 \%$ & $-7.1 \%$ \\
\hline & & & G2: Lower extremity aBMD & $\mathrm{N} / \mathrm{R}$ & $-2.3 \%$ & $-4.7 \%$ \\
\hline & & Training parameters: & & & & \\
\hline & & $\mathrm{G} 1: 2 \times 15 \mathrm{~min} / \mathrm{d}$ & G1: Femur neck aBMD & $N / R$ & $-4.5 \%$ & $-11.6 \%$ \\
\hline & & $\begin{array}{l}\text { 4:8 s on:off } \\
\text { G2. Inactive }\end{array}$ & G2: Femur neck aBMD & $\mathrm{N} / \mathrm{R}$ & $-3.2 \%$ & $-6.5 \%$ \\
\hline & & & G1: Femur proximal aBMD & $N / R$ & $-6.0 \%$ & $-11 \%$ \\
\hline & & & G2: Femur proximal aBMD & $N / R$ & $-3.7 \%$ & $-8.4 \%$ \\
\hline
\end{tabular}


Table 2. Cont.

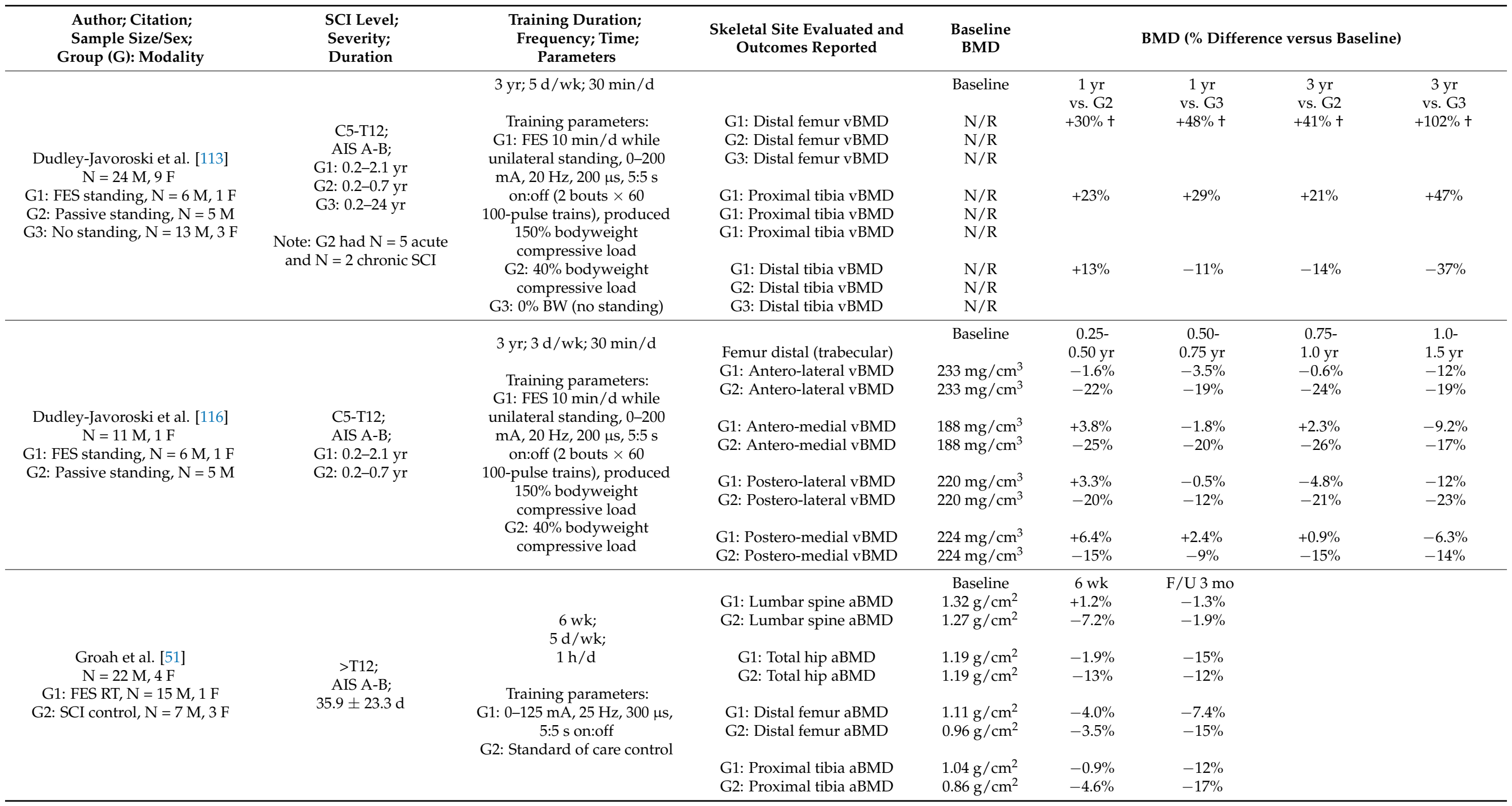


Table 2. Cont.

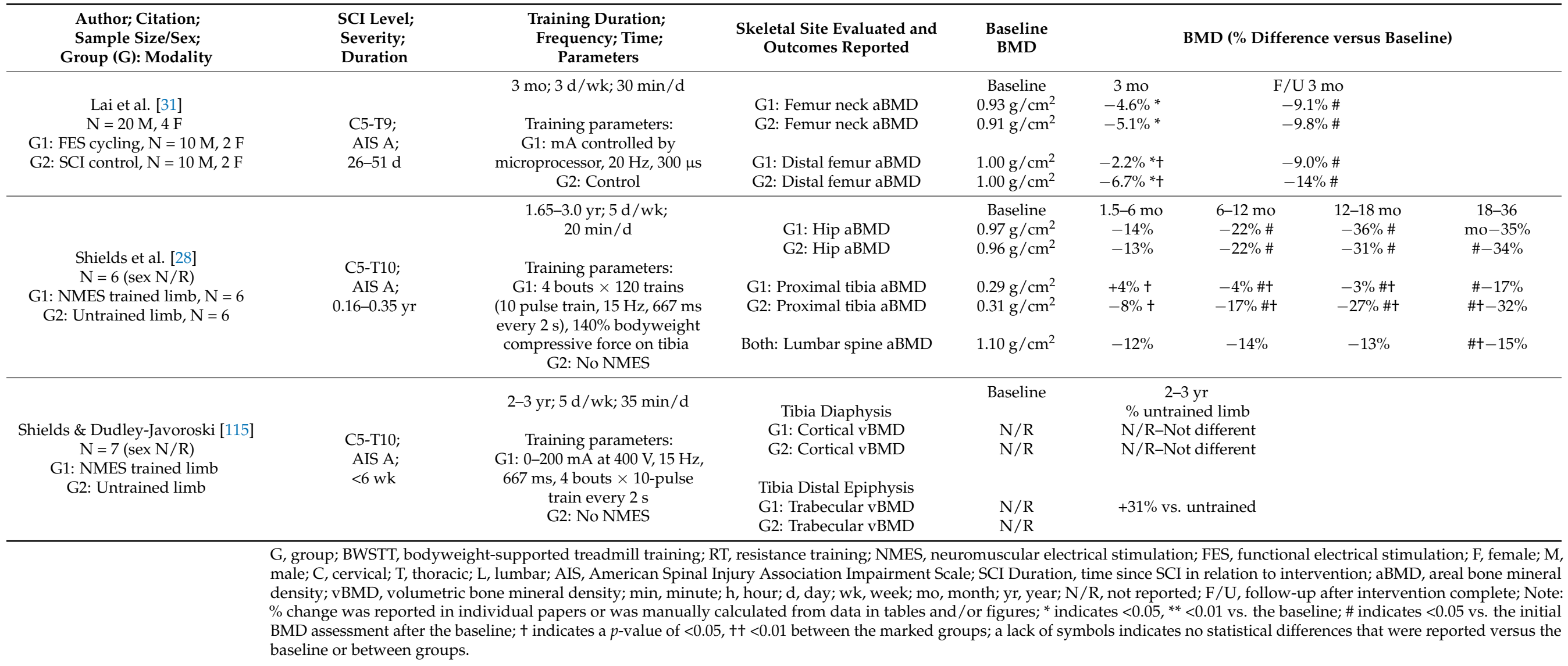


In comparison, 6 of the 11 controlled trials reported that an ABPT attenuated BMD loss at different sublesional sites [28,31,109,112,113,115]. Lai et al. [31] reported that 3-months of FES cycling attenuated aBMD loss at the distal femur $(-2.2 \%$ from the baseline) as compared to the controls ( $-6.7 \%$ from the baseline). Dudley-Javoroski et al. [113] reported that FES-mediated quadriceps contractions attenuated distal femur vBMD loss in the limb that was undergoing unilateral standing (25\% less bone loss at 1 -year and $39 \%$ less bone loss at three-years vs. the non-stimulated limbs), with a follow-up analysis confirming that FES attenuated trabecular vBMD loss in a roughly similar pattern in the anterior and posterior distal femur quadrants [116]. Shields et al. [28] also reported $\sim 50 \%$ less proximal tibia aBMD loss in the leg that underwent $>1.6$-years of NMES ( $-17 \%$ from the baseline) vs. the untrained leg of the same participant ( $-32 \%$ from the baseline). Additionally, three controlled trials reported attenuated BMD loss at sites other than the knee. Shields and Dudley-Javoroski [115] reported a 31\% higher trabecular vBMD at the distal tibia epiphysis in the limb that received FES strengthening 5 days/week for two- to three-years vs. the untrained limb and De Bruin et al. [112] reported that BWSTT or use of standing frames lessened distal tibia trabecular vBMD loss over six-months $(-0.4 \%$ from the baseline for both interventions) vs. the controls ( $-8 \%$ from the baseline). Lastly, Alekna et al. [109] reported that passive standing (>one hour/day, five days/week, for two-years) lessened aBMD loss vs. controls within the whole leg ( $-25 \%$ vs. $-34 \%$ from the baseline), and pelvis ( $-15 \%$ vs. $-21 \%$ from the baseline). Regardless, five of six controlled trials that observed attenuated BMD loss at the sites that were described above also reported no BMD differences in the treatment vs. control groups at other sites $[28,31,109,112,115]$. Of note, four of six trials that reported attenuated BMD loss utilized FES exercises [28,31,113,115] and two used standing exercise without FES [109,112]. However, a similar number of FES $[51,99,111]$ and standing trials $[110,114]$ observed no improvement in BMD at any site that was evaluated.

\section{ABPT Interventions and BMD after Chronic SCI}

A visual summary of studies examining the effects of different ABPT modalities at knee and non-knee sites in chronic SCI is presented in Figure 2A,B. Case studies or case series that enrolled participants with chronic SCI ranged from 10-weeks to 8-years in duration, instituted training frequencies of two- to five-days/week, and utilized the following modalities (Table 3): passive standing in a frame that was combined with leg or whole-body vibration [117], overground walking that was assisted by reciprocating gait orthosis [118], BWSTT alone [119] or in combination with epidural electrical stimulation [120] or nerve stimulation [121], or FES-based cycling [122,123] or rowing [124] that was delivered alone or after FES RT [106,125,126]. These reports produced inconsistent results with four case studies $[117,121,123,125]$ and one case series [118] observing an apparent BMD increase ranging from $2-20 \%$ vs. the baseline, three case studies [121,122,124] and one case series [120] reporting no clear bone change ( $<2 \%$ change vs. baseline), and two case studies [117,119] and three case series $[106,118,126]$ observing an apparent BMD reduction ranging from $2-21 \%$ vs. the baseline, depending on the site that was evaluated. Goktepe et al. [127] also reported no differences in T-scores among persons with chronic SCI who performed $\geq$ one hour/day or <one hour/day standing or no standing at several traditional osteoporosis sites. Of these studies, only two analyzed BMD changes at the knee. Lambach et al. [106] reported distal femur trabecular vBMD was $\sim 13 \%$ lower in two persons with chronic SCI after 9-12 months of FES RT and rowing, while Coupaud et al. [121] reported inconsistent distal femur and proximal tibia total and trabecular vBMD changes (range: $-2.8 \%$ to $+2.5 \%$ vs. the baseline) in a person with chronic SCI who underwent BWSTT with unilateral peroneal nerve stimulation for seven-months. Within a cross-sectional design, Gibbons et al. also reported that a person with chronic SCI displayed 31\% higher proximal tibia trabecular vBMD after completing eight-years of FES rowing [124] and 80-125\% higher total and trabecular vBMD at the distal tibia after 10-years, [15] when compared with a historical cohort of persons with chronic SCI, although, vBMD remained 7-19\% lower in 
this person compared with uninjured persons. Of note, three of five case studies/series that reported higher BMD vs. a baseline incorporated FES [123,125] or nerve stimulation [121], while four others that utilized FES reported no BMD change [122,124] or reduced BMD vs. the baseline $[106,126]$.

Inconsistent results also exist in uncontrolled and controlled trials that evaluated the effects of ABPT on bone in persons with chronic SCI. In total, we identified 14 trials without a control comparator group (Table 4), which ranged from 6-weeks to 16-months in duration with a training frequency of two- to seven-days/week and assessed: passive loading in a standing frame [128], standing that was combined with whole body vibration [102], BWSTT alone [100], over ground walking in an exoskeleton [129] or reciprocating gait orthosis [130], a multimodal ABPT regimen (described above) [103], or FES-based overground walking [131], RT [108], cycling [29,30,132], or a combination of RT and cycling [133-135]. Three uncontrolled trials reported increased BMD [29,30,134] vs. a baseline at some sublesional sites, while 10 reported no bone change at any sublesional site evaluated $[100,102,128-135]$ and one enrolling persons with acute and chronic SCI observed BMD reductions at most sites assessed [103]. In addition, we identified 10 controlled trials (Table 5) that ranged from 12-weeks to 12-months in duration, utilized a training frequency of three- to five-days/week and evaluated: seated vibration to the lower limbs [101,136], a combined protocol involving BWSTT, overground walking, and RT [97], or FES while undergoing BWSTT [98], RT [137,138], cycling [139], or RT and cycling $[96,140]$ or rowing [141]. A total of two controlled trials reported increased BMD vs. a baseline at select bone sites $[96,137]$ and two indicated higher BMD vs. a control group $[97,140]$, while five reported no difference vs. a baseline or a control group at any site evaluated [98,101,136,138,139] and one acquired but did not report BMD [141].

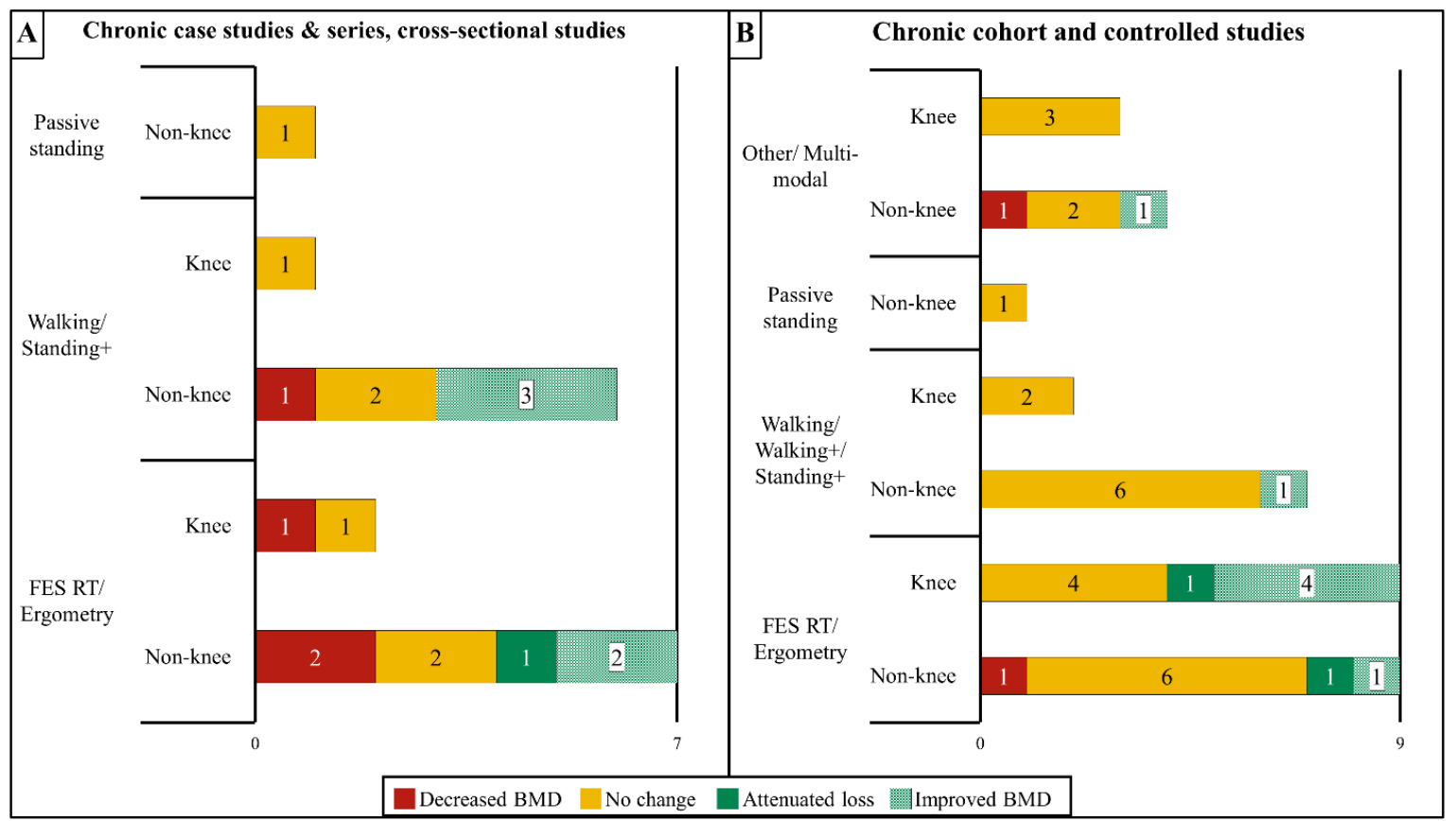

Figure 2. Summary of the effects of activity-based physical therapy (ABPT) on the changes in bone mineral density (BMD) at the knee (distal femur and proximal tibia) or all other sublesional non-knee sites for persons with chronic SCI in (A) case studies, case series, and cross-sectional studies, or (B) controlled and cohort studies. The data are reported as described in Figure 1A,B. Overall, most studies on persons with chronic SCI reported that ABPT did not alter BMD with only four studies reporting improved BMD at the knee. FES, functional electric stimulation; RT, resistance training; "Walking+" refers to interventions that combined walking ABPT with FES or nerve stimulation; "Standing+" refers to interventions that combined standing modalities with other modalities that increase muscle activation such as FES or vibration. 
Table 3. Case studies, case series, and cross-sectional studies evaluating the effects of activity-based physical therapy (ABPT) and/or loading on bone mineral density (BMD) or T-scores in adults with chronic spinal cord injury (SCI).

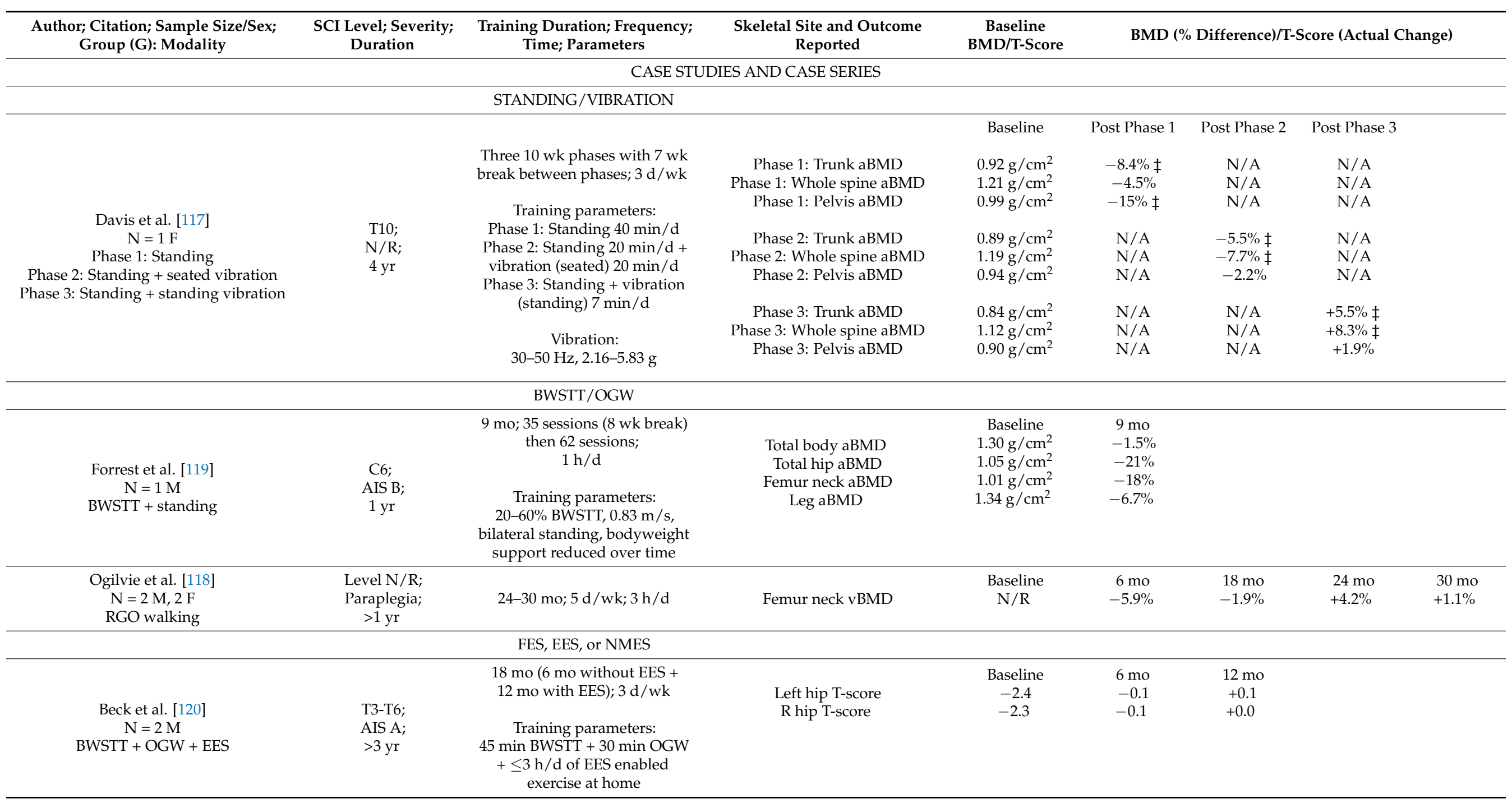


Table 3. Cont.

\begin{tabular}{|c|c|c|c|c|c|}
\hline $\begin{array}{l}\text { Author; Citation; Sample Size/Sex; } \\
\text { Group (G): Modality }\end{array}$ & $\begin{array}{l}\text { SCI Level; Severity; } \\
\text { Duration }\end{array}$ & $\begin{array}{c}\text { Training Duration; Frequency; } \\
\text { Time; Parameters }\end{array}$ & $\begin{array}{l}\text { Skeletal Site and Outcome } \\
\text { Reported }\end{array}$ & $\begin{array}{c}\text { Baseline } \\
\text { BMD/T-Score }\end{array}$ & BMD (\% Difference)/T-Score (Actual Change) \\
\hline \multirow{17}{*}{$\begin{array}{c}\text { Coupaud et al. [121] } \\
\mathrm{N}=1 \mathrm{M} \\
\text { BWSTT }+ \text { unilateral peroneal nerve } \\
\text { stimulation to left (L) side }\end{array}$} & \multirow{17}{*}{$\begin{array}{l}\text { T6; } \\
\text { AIS C } \\
14.5 \mathrm{yr}\end{array}$} & \multirow{17}{*}{$\begin{array}{c}\text { Training parameters: } \\
\text { 30\% BWSTT } \\
\text { Unilateral FES (L side) } \\
\text { peroneal nerve: } \\
40 \mathrm{~mA}, 40 \mathrm{~Hz}, 117-351 \mu \mathrm{s}\end{array}$} & Femur (vBMD) & Baseline & $7 \mathrm{mo}$ \\
\hline & & & $\mathrm{R}$ diaphysis cortical & $1103 \mathrm{mg} / \mathrm{cm}^{3}$ & $+0.5 \%$ \\
\hline & & & $\mathrm{L}$ diaphysis cortical & $1108 \mathrm{mg} / \mathrm{cm}^{3}$ & $+0.3 \%$ \\
\hline & & & $R$ distal epiphysis total & $198 \mathrm{mg} / \mathrm{cm}^{3}$ & $+1.2 \%$ \\
\hline & & & L distal epiphysis total & $191 \mathrm{mg} / \mathrm{cm}^{3}$ & $+1.1 \%$ \\
\hline & & & $R$ distal epiphysis trabecular & $159 \mathrm{mg} / \mathrm{cm}^{3}$ & $+2.2 \%$ \\
\hline & & & L distal epiphysis trabecular & $147 \mathrm{mg} / \mathrm{cm}^{3}$ & $+0.5 \%$ \\
\hline & & & & & \\
\hline & & & Tibia (vBMD) & & \\
\hline & & & R proximal epiphysis total & $136 \mathrm{mg} / \mathrm{cm}^{3}$ & $\begin{array}{l}+0.0 \% \\
+25 \%\end{array}$ \\
\hline & & & & & \\
\hline & & & $\mathrm{R}$ proximal epiphysis trabecular & $83.1 \mathrm{mg} / \mathrm{cm}^{3}$ & $+1.1 \%$ \\
\hline & & & L proximal epiphysis trabecular & $85.5 \mathrm{mg} / \mathrm{cm}^{3}$ & $-2.8 \%$ \\
\hline & & & $\mathrm{R}$ diaphysis cortical & $1122 \mathrm{mg} / \mathrm{cm}^{3}$ & $-0.3 \%$ \\
\hline & & & $\mathrm{L}$ diaphysis cortical & $1119 \mathrm{mg} / \mathrm{cm}^{3}$ & $-0.3 \%$ \\
\hline & & & $\mathrm{R}$ distal epiphysis trabecular & $118 \mathrm{mg} / \mathrm{cm}^{3}$ & $+4.9 \% \ddagger$ \\
\hline & & & L distal epiphysis trabecular & $108 \mathrm{mg} / \mathrm{cm}^{3}$ & $+20 \% \ddagger$ \\
\hline \multirow{7}{*}{$\begin{array}{c}\text { Deley et al. [125] } \\
\mathrm{N}=1 \mathrm{~F} \\
\text { FES RT + FES rowing }\end{array}$} & \multirow{7}{*}{$\begin{array}{l}\text { T4-T5; } \\
\text { AIS A; } \\
2 \mathrm{yr}\end{array}$} & $12 \mathrm{mo}$ (3 mo RT, 9 mo rowing); & \multirow{7}{*}{ Femur neck aBMD } & \multirow{7}{*}{$\begin{array}{l}\text { Baseline } \\
0.53 \mathrm{~g} / \mathrm{cm}^{2}\end{array}$} & \multirow{7}{*}{$\begin{array}{l}12 \mathrm{mo} \\
+19 \%\end{array}$} \\
\hline & & $3 \mathrm{~d} / \mathrm{wk}$ & & & \\
\hline & & & & & \\
\hline & & Training parameters: & & & \\
\hline & & RT: $0-110 \mathrm{~mA}, 40 \mathrm{~Hz}, 450 \mu \mathrm{s}$, & & & \\
\hline & & $6: 6 \mathrm{~s}$ on:off & & & \\
\hline & & Rowing: $40 \mathrm{~Hz}, 450 \mu \mathrm{s}$ & & & \\
\hline \multirow{5}{*}{$\begin{array}{c}\text { Dolbow et al. [123] } \\
\mathrm{N}=1 \mathrm{~F} \\
\text { FES cycling }\end{array}$} & \multirow{5}{*}{$\begin{array}{l}\text { T6; } \\
\text { AIS A; } \\
2 \mathrm{yr}\end{array}$} & $12 \mathrm{mo} ; 3 \mathrm{~d} / \mathrm{wk} ; 1 \mathrm{~h} / \mathrm{d}$ & \multirow{5}{*}{ Total body aBMD } & \multirow{5}{*}{$\begin{array}{l}\text { Baseline } \\
0.93 \mathrm{~g} / \mathrm{cm}^{2}\end{array}$} & \multirow{5}{*}{$\begin{array}{l}12 \mathrm{mo} \\
+9.5 \%\end{array}$} \\
\hline & & & & & \\
\hline & & Training parameters: & & & \\
\hline & & $140 \mathrm{~mA}, 33.3-50 \mathrm{~Hz}$ & & & \\
\hline & & $\begin{array}{l}250-300 \mu \mathrm{s}, 0.64-1.28 \mathrm{Nm} \text { avg } \\
\text { resistance, } 36-43 \mathrm{rpm}\end{array}$ & & & \\
\hline
\end{tabular}


Table 3. Cont.

\begin{tabular}{|c|c|c|c|c|c|c|c|}
\hline $\begin{array}{l}\text { Author; Citation; Sample Size/Sex; } \\
\text { Group (G): Modality }\end{array}$ & $\begin{array}{l}\text { SCI Level; Severity; } \\
\text { Duration }\end{array}$ & $\begin{array}{l}\text { Training Duration; Frequency; } \\
\text { Time; Parameters }\end{array}$ & $\begin{array}{l}\text { Skeletal Site and Outcome } \\
\text { Reported }\end{array}$ & $\begin{array}{l}\text { Baseline } \\
\text { BMD/T-Score }\end{array}$ & \multicolumn{3}{|c|}{ BMD (\% Difference)/T-Score (Actual Change) } \\
\hline $\begin{array}{c}\text { Dolbow et al. [122] } \\
\mathrm{N}=1 \mathrm{M} \\
\text { FES cycling }\end{array}$ & $\begin{array}{l}\text { C4; } \\
\text { AIS C; } \\
33 \mathrm{yr}\end{array}$ & $\begin{array}{c}56 \mathrm{mo} ; 3 \mathrm{~d} / \mathrm{wk} \\
40-60 \mathrm{~min} / \mathrm{d} \\
\\
\text { Training parameters: } \\
120-140 \mathrm{~mA}, 33.3-35.7 \mathrm{~Hz}, \\
250-300 \mu \mathrm{s}, 0.5-2.0 \mathrm{Nm} \text { avg } \\
\text { resistance, } 40-50 \mathrm{rpm}\end{array}$ & Total body aBMD & $\begin{array}{l}\text { Baseline } \\
1.02 \mathrm{~g} / \mathrm{cm}^{2}\end{array}$ & $\begin{array}{l}56 \mathrm{mo} \\
-0.6 \%\end{array}$ & & \\
\hline $\begin{array}{c}\text { Gibbons et al. [124] } \\
\quad \mathrm{N}=1 \mathrm{M} \\
\text { FES rowing, } \mathrm{N}=1 \mathrm{M}\end{array}$ & $\begin{array}{l}\text { G1: T4; } \\
\text { AIS A; } \\
13.5 \mathrm{yr}\end{array}$ & $\begin{array}{c}>8 \mathrm{yr} ; 2-4 \mathrm{~d} / \mathrm{wk} \\
15-45 \mathrm{~min} / \mathrm{d} \\
\\
\text { Training parameters: } \\
0-115 \mathrm{~mA}, 50 \mathrm{~Hz}, 450 \mu \mathrm{s}\end{array}$ & Total hip T-score & $\begin{array}{l}\text { T-score } \\
-2.3\end{array}$ & $\begin{array}{l}>8 \mathrm{yr} \\
+0.6\end{array}$ & $\begin{array}{r}\text { Proximal tib } \\
\text { reported }\end{array}$ & $\begin{array}{l}\text { Note: } \\
\text { trabecular vBMD values are } \\
\text { the cross-sectional study } \\
\text { ection below. }\end{array}$ \\
\hline $\begin{array}{c}\text { Lambach et al. [106] } \\
\mathrm{N}=4 \mathrm{M} \\
\text { FES KE } / \text { KF RT + FES rowing }\end{array}$ & $\begin{array}{l}\text { C7-T10; } \\
\text { AIS A-B; } \\
\text { 10-16 mo }\end{array}$ & $\begin{array}{c}\text { 9-12 mo (90 sessions); } \\
3 \mathrm{~d} / \mathrm{wk} ; \\
30-60 \mathrm{~min} / \mathrm{d} \\
\\
\text { Training parameters: } \\
\text { FES RT: } 0-120 \mathrm{~mA}, 40 \mathrm{~Hz} \\
450 \mu \mathrm{s}, 5 \mathrm{~s} \text { on: } 1 \mathrm{~s} \text { off } \\
\text { FES Rowing: N/R }\end{array}$ & $\begin{array}{c}\text { Entire cohort } \\
\text { Distal femur trabecular vBMD } \\
\text { Distal tibia trabecular vBMD } \\
\text { Chronic cohort } \\
\text { Distal femur trabecular vBMD } \\
\text { Distal tibia trabecular vBMD }\end{array}$ & $\begin{array}{c}\text { Baseline } \\
207 \mathrm{mg} / \mathrm{cm}^{3} \\
168 \mathrm{mg} / \mathrm{cm}^{3}\end{array}$ & $\begin{array}{c}30 \text { sessions } \\
\quad-8.4 \% \\
-14 \%\end{array}$ & $\begin{array}{c}60 \text { sessions } \\
-5.9 \% \\
-17 \%\end{array}$ & $\begin{array}{c}90 \text { sessions } \\
\\
-8.2 \% \\
-19 \%\end{array}$ \\
\hline
\end{tabular}

Note: Lambach et al. included N = $2<1 \mathrm{yr}$ and $\mathrm{N}=2$ chronic SCI. Baseline and \% difference values are reported for the entire cohort and the chronic SCI cohort. BMD values for the subacute cohort are in Table 1.
$10 \mathrm{wk} R T ; 5 \mathrm{~d} / \mathrm{wk} ; 15 \mathrm{~min} / \mathrm{d}$
Baseline
$0.16 \mathrm{~g} / \mathrm{cm}^{2}$
$42 \mathrm{wk}$
Distal tibia vBMD
$-3.2 \%$

then 32 wk cycle;
$5 \mathrm{~d} / \mathrm{wk} ; 15 \mathrm{~min} / \mathrm{d}$

Pacy et al. [126]

$\mathrm{N}=4 \mathrm{M}$

T4-T6;

FES KE RT + FES cycling
AIS A; $\quad$ Training parameters:

ES RT: $65-90 \mathrm{~V}, 40 \mathrm{~Hz}, 300 \mu \mathrm{s}$, $6: 6 \mathrm{~s}$ on:off

FES Cycle: 80-125 V, $50 \mathrm{rpm}$, 
Table 3. Cont.

\begin{tabular}{|c|c|c|c|c|c|c|}
\hline $\begin{array}{c}\text { Author; Citation; Sample Size/Sex; } \\
\text { Group (G): Modality }\end{array}$ & $\begin{array}{l}\text { SCI Level; Severity; } \\
\text { Duration }\end{array}$ & $\begin{array}{c}\text { Training Duration; Frequency; } \\
\text { Time; Parameters }\end{array}$ & $\begin{array}{l}\text { Skeletal Site and Outcome } \\
\text { Reported }\end{array}$ & $\begin{array}{c}\text { Baseline } \\
\text { BMD/T-Score }\end{array}$ & \multicolumn{2}{|c|}{ BMD (\% Difference)/T-Score (Actual Change) } \\
\hline & & & & Baseline & \multicolumn{2}{|c|}{ Actual T-score } \\
\hline & & & G1: L2-L4 T-score & $N / R$ & \multicolumn{2}{|c|}{-0.3} \\
\hline & & & G2: L2-L4 T-score & $N / R$ & \multicolumn{2}{|c|}{-0.2} \\
\hline & & & G3: L2-L4 T-score & $\mathrm{N} / \mathrm{R}$ & \multicolumn{2}{|c|}{+0.1} \\
\hline & & & G1: Femur neck T-score & $\mathrm{N} / \mathrm{R}$ & \multicolumn{2}{|c|}{-1.6} \\
\hline & & & G2: Femur neck T-score & $\mathrm{N} / \mathrm{R}$ & \multicolumn{2}{|c|}{-2.0} \\
\hline & & & G3: Femur neck T-score & $\mathrm{N} / \mathrm{R}$ & \multirow{2}{*}{\multicolumn{2}{|c|}{-2.0}} \\
\hline $\mathrm{N}=60 \mathrm{M}, 11 \mathrm{~F}$ & AIS A-B; & Duration N/R; daily; & & & & \\
\hline G1: Standing $>1 \mathrm{~h} / \mathrm{d}, \mathrm{N}=15 \mathrm{M}, 5 \mathrm{~F}$ & All groups: & $\mathrm{G} 1: \geq 1 \mathrm{~h} / \mathrm{d}$ & G1: Ward triangle T-score & $\mathrm{N} / \mathrm{R}$ & \multicolumn{2}{|c|}{-1.3} \\
\hline G2: Standing $<1 \mathrm{~h} / \mathrm{d}, \mathrm{N}=9 \mathrm{M}, 2 \mathrm{~F}$ & $\geq 1 \mathrm{yr}$ & G2: $<1 \mathrm{~h} / \mathrm{d}$ & G2: Ward triangle T-score & $\mathrm{N} / \mathrm{R}$ & \multicolumn{2}{|c|}{-1.5} \\
\hline G3: No standing, $\mathrm{N}=36 \mathrm{M}, 4 \mathrm{~F}$ & $(4.41 \pm 2.99 \mathrm{yr})$ & G3: No standing & G3: Ward triangle T-score & $N / R$ & \multicolumn{2}{|c|}{-1.5} \\
\hline & & & G1: Femur trochanter T-score & $\mathrm{N} / \mathrm{R}$ & \multicolumn{2}{|c|}{-2.3} \\
\hline & & & G2: Femur trochanter T-score & $N / R$ & \multirow{2}{*}{\multicolumn{2}{|c|}{$\begin{array}{l}-2.2 \\
-2.5\end{array}$}} \\
\hline & & & G3: Femur trochanter T-score & $N / R$ & & \\
\hline & & & G1: Total femur T-score & $N / R$ & \multicolumn{2}{|c|}{-2.1} \\
\hline & & & G2: Total femur T-score & $N / R$ & \multirow{2}{*}{\multicolumn{2}{|c|}{$\begin{array}{l}-2.3 \\
-2.4\end{array}$}} \\
\hline & & & G3: Total femur T-score & $\mathrm{N} / \mathrm{R}$ & & \\
\hline & G1: T4; & \multirow[b]{2}{*}{$>8 \mathrm{yr} ; 2-4 \mathrm{~d} / \mathrm{wk} ; 15-45 \mathrm{~min} / \mathrm{d}$} & \multirow{5}{*}{ Proximal tibia trabecular vBMD } & Baseline & \multirow{5}{*}{$\begin{array}{l}\text { vs. G2 } \\
+31 \%\end{array}$} & \multirow{5}{*}{$\begin{array}{l}\text { vs. G3 } \\
-19 \%\end{array}$} \\
\hline $\begin{array}{l}\text { Gibbons et al. [124] } \\
\qquad N=1 \mathrm{M}\end{array}$ & $\begin{array}{l}\text { AIS A; } \\
13.5 \mathrm{vr}\end{array}$ & & & $\mathrm{N} / \mathrm{R}$ & & \\
\hline G1: FES rowing, $\mathrm{N}=1 \mathrm{M}$ & & \multirow{3}{*}{$\begin{array}{l}\text { Training parameters: } \\
0-115 \mathrm{~mA}, 50 \mathrm{~Hz}, 450 \mu \mathrm{s}\end{array}$} & & & & \\
\hline G2: Historical SCI cohort, $\mathrm{N}=9$ & G2: C6-T10; & & & & & \\
\hline G3: Normative non-SCI, N = 14 & $\begin{array}{c}\text { AIS A; } \\
6.6 \pm 2.8 \mathrm{yr}\end{array}$ & & & & & \\
\hline \multirow{6}{*}{$\begin{array}{c}\text { Gibbons et al. [15] } \\
\mathrm{N}=1 \mathrm{M} \\
\text { G1: FES rowing, } \mathrm{N}=1 \mathrm{M} \\
\text { G2: Historical SCI cohort, } \mathrm{N}=9 \\
\text { G3: Normative non-SCI, } \mathrm{N}=22\end{array}$} & G1: T4; & \multirow{3}{*}{$>10 \mathrm{yr} ; 3 \mathrm{~d} / \mathrm{wk} ; 30 \mathrm{~min} / \mathrm{d}$} & & Baseline & \multirow{6}{*}{$\begin{array}{l}\text { vs. G2 } \\
+82 \% \\
+125 \%\end{array}$} & \multirow{6}{*}{$\begin{array}{l}\text { vs. G3 } \\
-6.5 \% \\
-15 \%\end{array}$} \\
\hline & AIS A; & & Distal tibia total vBMD & $\mathrm{N} / \mathrm{R}$ & & \\
\hline & $14 \mathrm{yr}$ & & Distal tibia trabecular vBMD & $\mathrm{N} / \mathrm{R}$ & & \\
\hline & & Training parameters: & & & & \\
\hline & $\begin{array}{l}\text { G2: T3-T12; } \\
\text { AIS A-B; }\end{array}$ & $0-115 \mathrm{~mA}, 50 \mathrm{~Hz}, 450 \mu \mathrm{s}$ & & & & \\
\hline & $11.4 \pm 9.4 \mathrm{yr}$ & & & & & \\
\hline
\end{tabular}

G, group; BWSTT, bodyweight-supported treadmill training: RGO, reciprocating gait orthosis; OGW, overground walking; EES, epidural electrical stimulation; NMES, neuromuscular

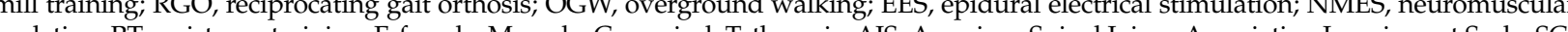
election, Duration: tin s. average; min, minute; $h$, hour; $d$, day; wk, week; mo, month; $y r$, year; $\mathrm{m}$, meter; $\mathrm{N} / \mathrm{R}$, not reported. Note: \% change was reported in individual papers or was manually calculated from data in tables and/or figures; $\ddagger$ indicates exceeded least significant change; lack of symbols indicates no statistical differences that were reported versus baseline or between the groups. 
Table 4. Uncontrolled interventional studies evaluating the effects of activity-based physical therapy (ABPT) and/or loading on bone mineral density (BMD) or $\mathrm{T}$-scores in adults with chronic spinal cord injury (SCI).

\begin{tabular}{|c|c|c|c|c|c|c|c|}
\hline \multirow{2}{*}{$\begin{array}{l}\text { Author; Citation; } \\
\text { Sample Size/Sex; } \\
\text { Modality }\end{array}$} & \multirow[t]{2}{*}{$\begin{array}{c}\text { SCI Level; } \\
\text { Severity; Duration }\end{array}$} & \multirow{2}{*}{$\begin{array}{c}\begin{array}{c}\text { Training Duration; } \\
\text { Frequency; Time; } \\
\text { Parameters }\end{array} \\
\text { STANDING/VIBRATION }\end{array}$} & \multirow[t]{2}{*}{ Bone Site Evaluated } & \multirow[t]{2}{*}{$\begin{array}{c}\text { Baseline } \\
\text { BMD/T-Score }\end{array}$} & \multicolumn{3}{|c|}{ BMD (\% Difference) T-Score (Actual Change } \\
\hline & & & & & & & \\
\hline $\begin{array}{c}\text { Kunkel et al. [128] } \\
\mathrm{N}=6 \mathrm{M} \\
\text { Standing }\end{array}$ & $\begin{array}{l}\text { C5-T12; } \\
\text { Incomplete and } \\
\text { complete; } \\
10-39 \mathrm{yr}\end{array}$ & $\begin{array}{l}6 \text { mo (mean } \sim 135 \mathrm{~d} \text { ); } \\
45 \text { min twice daily } \\
\text { Training parameters: } \\
\text { Standing frame }\end{array}$ & $\begin{array}{l}\text { Lumbar spine aBMD } \\
\text { Femur neck aBMD }\end{array}$ & $\begin{array}{c}\text { Baseline } \\
1.26 \mathrm{~g} / \mathrm{cm}^{2} \\
0.51 \mathrm{~g} / \mathrm{cm}^{2}\end{array}$ & $\begin{array}{l}3 \mathrm{mo} \\
-1.6 \% \\
+18 \%\end{array}$ & $\begin{array}{l}6 \mathrm{mo} \\
-5.6 \% \\
+9.8 \%\end{array}$ & \\
\hline $\begin{array}{l}\text { Wuermser et al. [102] } \\
\mathrm{N}=5 \mathrm{M}, 4 \mathrm{~F} \\
\text { Standing + vibration }\end{array}$ & $\begin{array}{l}\text { AIS A-B; } \\
\text { T3-T12; } \\
2-27 \text { yr }\end{array}$ & $\begin{array}{c}6 \mathrm{mo} ; 5 \mathrm{~d} / \mathrm{wk} ; 20 \mathrm{~min} / \mathrm{d} \\
\text { Training parameters: } \\
0.3 \mathrm{~g}, 34 \mathrm{~Hz} \text { sinusoidal } \\
\text { movement of } 50 \mu \mathrm{m} \mathrm{w} / \text { lower } \\
\text { extremities supporting } \sim 86 \% \\
\text { body weight }\end{array}$ & $\begin{array}{c}\text { Total hip aBMD } \\
\text { Femur neck aBMD } \\
\text { Distal tibia total vBMD } \\
\text { Distal tibia trabecular vBMD } \\
\text { Distal tibia cortical vBMD }\end{array}$ & $\begin{array}{c}\text { Baseline } \\
0.71 \mathrm{~g} / \mathrm{cm}^{2} \\
0.75 \mathrm{~g} / \mathrm{cm}^{2} \\
\\
168 \mathrm{~g} / \mathrm{cm}^{3} \\
67.5 \mathrm{~g} / \mathrm{cm}^{3} \\
810 \mathrm{~g} / \mathrm{cm}^{3}\end{array}$ & $\begin{array}{l}3 \mathrm{mo} \\
+0.0 \% \\
-1.3 \% \\
-2.8 \% \\
-2.0 \% \\
-2.6 \%\end{array}$ & $\begin{array}{l}6 \mathrm{mo} \\
+1.5 \% \\
+1.4 \% \\
-3.1 \% \\
-6.5 \% \\
-0.9 \%\end{array}$ & $\begin{array}{c}\mathrm{F} / \mathrm{U} \\
6 \mathrm{mo} \\
+2.9 \% \\
+2.7 \% \\
\\
-5.0 \% \\
-7.4 \% \\
-2.2 \%\end{array}$ \\
\hline & & BWSTT/OGW & & & & & \\
\hline $\begin{array}{c}\text { Giangregorio et al. [100] } \\
\mathrm{N}=11 \mathrm{M}, 2 \mathrm{~F} \\
\text { BWSTT }\end{array}$ & $\begin{array}{l}\text { C4-T12; } \\
\text { AIS B-C; } \\
1.2-24 \mathrm{yr}\end{array}$ & $\begin{array}{c}12 \text { mo ( } 144 \text { sessions); } \\
3 \mathrm{~d} / \mathrm{wk} ; \\
\leq 3 \text { bouts of } 5-50 \text { min each } \\
\text { Training parameters: } \\
\text { 0-80\% bodyweight support, } \\
\text { progressively reduced }\end{array}$ & $\begin{array}{c}\text { Whole body aBMD } \\
\text { Lumbar spine aBMD } \\
\text { Proximal femur total aBMD } \\
\text { Right distal femur total aBMD } \\
\text { Right proximal tibia total aBMD } \\
\\
\text { Femur diaphysis total vBMD } \\
\text { Femur diaphysis cortical vBMD } \\
\text { Tibial diaphysis total vBMD } \\
\text { Tibial diaphysis cortical vBMD }\end{array}$ & $\begin{array}{c}\text { Baseline } \\
1.12 \mathrm{~g} / \mathrm{cm}^{2} \\
\mathrm{~N} / \mathrm{R} \\
\mathrm{N} / \mathrm{R} \\
\mathrm{N} / \mathrm{R} \\
\mathrm{N} / \mathrm{R} \\
\\
770 \mathrm{~g} / \mathrm{cm}^{3} \\
848 \mathrm{~g} / \mathrm{cm}^{3} \\
745 \mathrm{~g} / \mathrm{cm}^{3} \\
851 \mathrm{~g} / \mathrm{cm}^{3}\end{array}$ & $\begin{array}{c}12 \mathrm{mo} \\
-2.2 \% \\
\mathrm{~N} / \mathrm{R} \\
-0.2 \% \\
+1.7 \% \\
+1.4 \% \\
\\
-1.6 \% \\
-0.8 \% \\
-2.3 \% \\
-2.0 \%\end{array}$ & & \\
\hline $\begin{array}{c}\text { Karelis et al. [129] } \\
\mathrm{N}=4 \mathrm{M}, 1 \mathrm{~F} \\
\text { Exoskeleton walking }\end{array}$ & $\begin{array}{l}\text { C7-T10; } \\
\text { AIS A; } \\
7.6 \pm 4.6 \mathrm{yr}\end{array}$ & $\begin{array}{c}6 \mathrm{wk} ; 3 \mathrm{~d} / \mathrm{wk} ; 60 \mathrm{~min} / \mathrm{d} \\
\text { Training parameters: } \\
\text { Walking: } 27.0 \pm 5.4 \mathrm{~min} / \mathrm{d} \\
\text { Standing: } 48.4 \pm 7.4 \mathrm{~min} / \mathrm{d}\end{array}$ & $\begin{array}{c}\text { Total body aBMD } \\
\text { Leg aBMD } \\
\text { Tibia diaphysis vBMD }\end{array}$ & $\begin{array}{c}\text { Baseline } \\
1.19 \mathrm{~g} / \mathrm{cm}^{2} \\
1.11 \mathrm{~g} / \mathrm{cm}^{2} \\
\\
466 \mathrm{mg} / \mathrm{cm}^{3}\end{array}$ & $\begin{array}{l}6 \mathrm{wk} \\
-1.7 \% \\
+0.5 \% \\
+14 \%\end{array}$ & & \\
\hline
\end{tabular}


Table 4. Cont.

\begin{tabular}{|c|c|c|c|c|c|c|c|}
\hline $\begin{array}{l}\text { Author; Citation; } \\
\text { Sample Size/Sex; }\end{array}$ & $\begin{array}{l}\text { SCI Level; } \\
\text { Severity; }\end{array}$ & $\begin{array}{l}\text { Training Duration; } \\
\text { Frequency; Time; }\end{array}$ & Bone Site Evaluated & $\begin{array}{c}\text { Baseline } \\
\text { BMD/T-Score }\end{array}$ & \multicolumn{3}{|c|}{ BMD (\% Difference) T-Score (Actual Change) } \\
\hline $\begin{array}{c}\text { Thoumie et al. [130] } \\
\mathrm{N}=6 \mathrm{M}, 1 \mathrm{~F} \\
\text { RGO walking }\end{array}$ & $\begin{array}{c}\text { T2-T10; } \\
\text { Severity N/R; } \\
\text { 15-60 mo }\end{array}$ & $\begin{array}{c}16 \text { mo; } 3 \mathrm{~d} / \mathrm{wk} ; 2 \mathrm{~h} / \mathrm{d} \\
\text { Training parameters: } \\
\text { RGO walking }\end{array}$ & $\begin{array}{l}\text { Lumbar spine aBMD } \\
\text { Femur neck aBMD }\end{array}$ & $\begin{array}{l}\text { Z-score } \\
0.77 \\
1.02\end{array}$ & $\begin{array}{l}16 \mathrm{mo} \\
+0.01 \\
-0.06\end{array}$ & \multicolumn{2}{|c|}{$\begin{array}{l}\text { Note: Z-score was reported without BMD or } \\
\text { T-score values }\end{array}$} \\
\hline & & MULTIMODAL & & & & & \\
\hline $\begin{array}{c}\text { Astorino et al. [103] } \\
\mathrm{N}=11 \mathrm{M}, 2 \mathrm{~F} \\
\text { Multimodal ABPT regimen }\end{array}$ & $\begin{array}{l}\text { C4-L1; } \\
\text { Complete and } \\
\text { Incomplete; } \\
1.9 \pm 2.7 \mathrm{yr} \\
(0.2-9.3 \mathrm{yr})\end{array}$ & $\begin{array}{c}6 \mathrm{mo} \\
>2 \mathrm{~d} / \mathrm{wk} ; \\
2-3 \mathrm{~h} / \mathrm{d} \\
\\
\text { Training parameters: } \\
\text { Individualized protocol that included } \\
\text { active assistive exercise, lower } \\
\text { extremity and core RT, upper } \\
\text { extremity cycle ergometry, } \\
\text { bodyweight supported elliptical } \\
\text { training, BWSTT, OGW, vibration } \\
\text { training, and/or FES cycling }\end{array}$ & $\begin{array}{c}\text { Total body aBMD } \\
\text { Lumbar spine aBMD } \\
\text { R Total hip aBMD } \\
\text { L Total hip aBMD } \\
\text { R Femur neck aBMD } \\
\text { L Femur neck aBMD } \\
\text { R Femur trochanter aBMD } \\
\text { L Femur trochanter aBMD } \\
\text { R Distal femur aBMD } \\
\text { L Distal femur aBMD } \\
\text { R Proximal tibia aBMD } \\
\text { L Proximal tibia aBMD }\end{array}$ & $\begin{array}{c}\text { Baseline } \\
1.24 \mathrm{~g} / \mathrm{cm}^{2} \\
\\
1.27 \mathrm{~g} / \mathrm{cm}^{2} \\
\\
0.96 \mathrm{~g} / \mathrm{cm}^{2} \\
0.99 \mathrm{~g} / \mathrm{cm}^{2} \\
\\
0.98 \mathrm{~g} / \mathrm{cm}^{2} \\
0.99 \mathrm{~g} / \mathrm{cm}^{2} \\
\\
0.75 \mathrm{~g} / \mathrm{cm}^{2} \\
0.79 \mathrm{~g} \cdot \mathrm{cm}^{2} \\
\\
0.92 \mathrm{~g} / \mathrm{cm}^{2} \\
0.92 \mathrm{~g} / \mathrm{cm}^{2} \\
\\
0.88 \mathrm{~g} / \mathrm{cm}^{2} \\
0.89 \mathrm{~g} / \mathrm{cm}^{2}\end{array}$ & $\begin{array}{c}3 \mathrm{mo} \\
-1.6 \% \text { * } \\
+3.2 \% \text { * } \\
-3.1 \% \text { * } \\
-4.0 \% \text { * } \\
-2.0 \% \text { * } \\
-3.0 \% \text { * } \\
-2.7 \% \\
-5.1 \% \text { * } \\
-5.4 \% \\
-4.4 \% \\
-3.4 \% \\
-4.5 \%\end{array}$ & $\begin{array}{c}6 \mathrm{mo} \\
-2.5 \% \text { * } \\
+4.7 \% \text { * } \\
-6.3 \% \text { *\# } \\
-7.1 \% \text { *\# } \\
-5.1 \% \text { *\# } \\
-4.0 \% \text { * } \\
-6.7 \% \text { *\# } \\
-8.9 \% \text { * } \\
-11 \% \\
-7.6 \% \\
-8.0 \% \\
-11 \%\end{array}$ & $\begin{array}{c}\text { Note: BMD determined on } \\
\mathrm{N}=8<1 \mathrm{yr} \text { SCI and } \mathrm{N}=5 \\
\text { chronic SCI. Values are an } \\
\text { average of the total cohort } \\
\text { and were not determined } \\
\text { separately for acute and } \\
\text { chronic SCI cohorts. }\end{array}$ \\
\hline \multicolumn{8}{|c|}{ FES } \\
\hline $\begin{array}{c}\text { BeDell et al. [133] } \\
\mathrm{N}=12 \mathrm{M} \\
\text { Phase 1: FES KE RT } \\
\text { Phase 2: FES cycling progression } \\
\text { Phase 3a: FES cycling } \\
\text { Phase 3b: FES cycling + arm } \\
\text { ergometry }\end{array}$ & $\begin{array}{l}\text { C5-T12; } \\
\text { AIS A; } \\
9.7 \pm 5.1 \mathrm{yr} \\
(3-19 \mathrm{yr})\end{array}$ & $\begin{array}{c}\text { Phase } 1-3 \mathrm{a}: 34 \pm 8 \mathrm{wk} ; 3 \mathrm{~d} / \mathrm{wk} \\
\text { (actual } 2.0 \pm 0.3 \mathrm{~d} / \mathrm{wk}) ; 30 \mathrm{~min} / \mathrm{d} \\
\text { Phase } 3 \mathrm{~b}: 25 \pm 9 \mathrm{wk} ; \\
3 \mathrm{~d} / \mathrm{wk} ; 30 \mathrm{~min} / \mathrm{d} \\
\\
\text { Training parameters: } \\
\text { 10-132 } \mathrm{mA}, 30 \mathrm{~Hz}, 400 \mathrm{\mu s}\end{array}$ & $\begin{array}{c}\text { Lumbar 2-4 aBMD } \\
\text { Femur neck aBMD } \\
\text { Ward's triangle aBMD } \\
\text { Femur trochanter aBMD }\end{array}$ & $\begin{array}{c}\text { Baseline } \\
1.27 \mathrm{~g} / \mathrm{cm}^{2} \\
0.78 \mathrm{~g} / \mathrm{cm}^{2} \\
0.71 \mathrm{~g} / \mathrm{cm}^{2} \\
0.61 \mathrm{~g} / \mathrm{cm}^{2}\end{array}$ & $\begin{array}{l}\text { Phase 3a } \\
+1.6 \% \\
+1.3 \% \\
+0.0 \% \\
+4.9 \%\end{array}$ & $\begin{array}{l}\text { Phase } 3 \mathrm{~b} \\
+5.5 \% \\
+5.1 \% \\
-1.4 \% \\
+0.0 \%\end{array}$ & \\
\hline
\end{tabular}


Table 4. Cont.

\begin{tabular}{|c|c|c|c|c|c|c|}
\hline $\begin{array}{l}\text { Author; Citation; } \\
\text { Sample Size/Sex; } \\
\text { Modality }\end{array}$ & $\begin{array}{l}\text { SCI Level; } \\
\text { Severity; } \\
\text { Duration }\end{array}$ & $\begin{array}{l}\text { Training Duration; } \\
\text { Frequency; Time; } \\
\text { Parameters }\end{array}$ & Bone Site Evaluated & $\begin{array}{c}\text { Baseline } \\
\text { BMD/T-Score }\end{array}$ & \multicolumn{2}{|c|}{ BMD (\% Difference) T-Score (Actual Change) } \\
\hline $\begin{array}{c}\text { Chen et al. [29] } \\
\mathrm{N}=15 \mathrm{M} \\
\text { FES cycling, } \mathrm{N}=15 \mathrm{M}\end{array}$ & $\begin{array}{l}\text { C5-T8; } \\
\text { AIS A; } \\
2.6-15.7 \mathrm{yr}\end{array}$ & $\begin{array}{c}6 \mathrm{mo} \\
5 \mathrm{~d} / \mathrm{wk} \\
30 \mathrm{~min} / \mathrm{d} \\
\\
\text { Training parameters: } \\
0-120 \mathrm{~mA}, 20 \mathrm{~Hz}, 300 \mu \mathrm{s}\end{array}$ & $\begin{array}{c}\text { L2-L4 spine aBMD } \\
\text { Femur neck aBMD } \\
\text { Distal femur aBMD } \\
\text { Proximal tibia aBMD } \\
\text { Heel aBMD }\end{array}$ & $\begin{array}{l}\text { Baseline } \\
1.05 \mathrm{~g} / \mathrm{cm}^{2} \\
0.69 \mathrm{~g} / \mathrm{cm}^{2} \\
0.72 \mathrm{~g} / \mathrm{cm}^{2} \\
0.55 \mathrm{~g} / \mathrm{cm}^{2} \\
0.35 \mathrm{~g} . \mathrm{cm}^{2}\end{array}$ & $\begin{array}{l}6 \mathrm{mo} \\
+0.1 \% \\
-2.2 \% \\
+11 \% * \\
+13 \% * \\
+3.2 \%\end{array}$ & $\begin{array}{l}\mathrm{F} / \mathrm{U} 6 \mathrm{mo} \\
\quad+0.1 \% \\
-8.7 \% \# \\
-1.4 \% \# \\
-1.6 \% \# \\
-6.6 \% \#\end{array}$ \\
\hline $\begin{array}{c}\text { Frotzler et al. [134] } \\
\mathrm{N}=10 \mathrm{M}, 2 \mathrm{~F} \\
\text { FES RT + cycle }\end{array}$ & $\begin{array}{l}\text { T3-T12; } \\
\text { AIS A; } \\
\text { 3.6-25.5 yr }\end{array}$ & $\begin{array}{l}12 \mathrm{mo} \text { (actual: } 19 \pm 2.1 \mathrm{mo} \text { ); } \\
\text { FES RT: } 3-4 \mathrm{~d} / \text { wk for } 3 \mathrm{mo} \\
30-60 \mathrm{~min} / \mathrm{d} \text {; FES cycle: } \\
5 \mathrm{~d} / \text { wk for } 9 \text { mo; } 60 \mathrm{~min} / \mathrm{d} \\
\text { Training parameters: } \\
\text { FES RT: } 80-150 \mathrm{~mA}, 50 \mathrm{~Hz} \text {, } \\
\text { 300-400 } \mu \mathrm{s}, 5 \mathrm{~s} \text { on } / \text { off } \\
\\
\text { FES cycles: } 50 \mathrm{~Hz}, 0-500 \mu \mathrm{s}\end{array}$ & $\begin{array}{c}\text { Femur } \\
\text { Diaphysis cortical vBMD } \\
\text { Distal epiphysis total vBMD } \\
\text { Distal epiphysis trabecular vBMD } \\
\text { Tibia } \\
\text { Proximal total vBMD } \\
\text { Proximal trabecular vBMD } \\
\text { Diaphysis cortical vBMD } \\
\text { Distal total vBMD } \\
\text { Distal trabecular vBMD }\end{array}$ & $\begin{array}{l}124 \mathrm{mg} / \mathrm{cm}^{3} \\
71.6 \mathrm{mg} / \mathrm{cm}^{3} \\
1112 \mathrm{mg} / \mathrm{cm}^{3} \\
166 \mathrm{mg} / \mathrm{cm}^{3} \\
101 \mathrm{mg} / \mathrm{cm}^{3}\end{array}$ & $\begin{array}{l}-0.7 \% \\
-2.8 \% \\
+0.7 \% \\
-0.7 \% \\
-0.8 \%\end{array}$ & $\begin{array}{l}12 \mathrm{mo} \\
-0.4 \% \text { * } \\
+6.7 \% \text { * } \\
+13 \% \text { * }\end{array}$ \\
\hline $\begin{array}{l}\text { Griffin et al. [132] } \\
\mathrm{N}=13 \mathrm{M}, 5 \mathrm{~F} \\
\text { FES cycling }\end{array}$ & $\begin{array}{l}\text { C4-T7; } \\
\text { Complete- } \\
\text { Incomplete; } \\
2-53 \mathrm{yr} \\
\end{array}$ & $\begin{array}{c}10 \mathrm{wk} ; 2-3 \mathrm{~d} / \mathrm{wk} ; 30 \mathrm{~min} / \mathrm{d} \\
\text { Training parameters: } \\
0-140 \mathrm{~mA}, 50 \mathrm{~Hz}, 49 \mathrm{rpm}\end{array}$ & Total body bone mass & $\begin{array}{l}\text { Baseline } \\
6.03 \mathrm{lbs}\end{array}$ & $\begin{array}{r}10 \mathrm{wk} \\
-0.66 \%\end{array}$ & $\begin{array}{l}\text { Note: Data are bone mass (lbs), } \\
\text { BMD not reported. }\end{array}$ \\
\hline $\begin{array}{c}\text { Leeds et al. [135] } \\
\mathrm{N}=6 \mathrm{M} \\
\text { FES KE RT + FES cycling }\end{array}$ & $\begin{array}{l}\text { C4-C6; } \\
\text { AIS A; } \\
2-9 \mathrm{yr}\end{array}$ & $\begin{array}{c}1 \mathrm{mo} \mathrm{RT} ; 3 \mathrm{~d} / \mathrm{wk} ; \text { up to } 45 \mathrm{KE} \\
\text { then } 6 \text { mo cycle; } 3 \mathrm{~d} / \mathrm{wk} ; \\
\text { up to } 30 \mathrm{~min} / \mathrm{d} \\
\text { Training parameters: } \\
0-130 \mathrm{~mA}, \leq 220 \mathrm{~V}, 30-60 \mathrm{~Hz}, 350 \mathrm{\mu s}\end{array}$ & $\begin{array}{l}\text { Femur neck aBMD } \\
\text { Ward's triangle aBMD } \\
\text { Trochanter aBMD }\end{array}$ & $\begin{array}{l}\text { Baseline } \\
0.65 \mathrm{~g} / \mathrm{cm}^{2} \\
0.52 \mathrm{~g} / \mathrm{cm}^{2} \\
0.46 \mathrm{~g} / \mathrm{cm}^{2}\end{array}$ & $\begin{array}{c}6 \mathrm{mo} \\
-1.5 \% \\
+0.0 \% \\
-6.5 \%\end{array}$ & \\
\hline $\begin{array}{l}\text { Mohr et al. [30] } \\
\mathrm{N}=8 \mathrm{M}, 2 \mathrm{~F} \\
\text { FES cycle }\end{array}$ & $\begin{array}{l}\text { C6-T4; } \\
\text { Complete; } \\
12.5 \pm 2.7 \mathrm{yr} \\
(2-24 \mathrm{yr})\end{array}$ & $\begin{array}{c}3 \mathrm{~d} / \mathrm{wk} \text { for } 12 \mathrm{mo} \text {, then } 1 \mathrm{~d} / \mathrm{wk} \text { for } \\
6 \mathrm{mo} ; 30 \mathrm{~min} / \mathrm{d} \\
\text { Training parameters: } \\
0-130 \mathrm{~mA}, 30 \mathrm{~Hz}, 350 \mathrm{~ms}, 50 \mathrm{rpm}\end{array}$ & $\begin{array}{l}\text { Femur neck aBMD } \\
\text { Lumbar spine aBMD } \\
\text { Proximal tibia aBMD }\end{array}$ & $\begin{array}{l}\text { Baseline } \\
0.63 \mathrm{~g} / \mathrm{cm}^{2} \\
1.21 \mathrm{~g} / \mathrm{cm}^{2} \\
0.49 \mathrm{~g} / \mathrm{cm}^{2}\end{array}$ & $\begin{array}{l}12 \mathrm{mo} \\
-3.2 \% \\
+0.8 \% \\
+10 \%\end{array}$ & $\begin{array}{l}18 \mathrm{mo} \\
-13 \% \\
+1.7 \% \\
-2.0 \%\end{array}$ \\
\hline $\begin{array}{c}\text { Needham-Shropshire et al. [131] } \\
\text { N = 13 M, } 3 \text { F } \\
\text { FES + OGW }\end{array}$ & $\begin{array}{l}\text { T4-T11; } \\
\text { AIS A; } \\
>6 \text { mo } \\
3.8 \text { yrs (avg) }\end{array}$ & $\begin{array}{l}20 \mathrm{wk} ; 3 \mathrm{~d} / \mathrm{wk} ; \leq 120 \mathrm{~min} / \mathrm{d} \\
\text { Training parameters: } \\
0-300 \mathrm{~mA}, 24 \mathrm{~Hz}, 150300 \mu \mathrm{s}\end{array}$ & $\begin{array}{l}\text { G1: Femur neck aBMD } \\
\text { G1: Ward's triangle aBMD } \\
\text { G1: Femur Trochanter aBMD }\end{array}$ & $\begin{array}{c}\text { Baseline } \\
0.77 \mathrm{~g} / \mathrm{cm}^{2} \\
0.69 \mathrm{~g} / \mathrm{cm}^{2} \\
0.58 \mathrm{~g} / \mathrm{cm}^{2}\end{array}$ & $\begin{array}{l}12 \mathrm{wk} \\
-1.3 \% \\
-1.5 \% \\
-1.7 \%\end{array}$ & $\begin{array}{c}20 \mathrm{wk} \\
-1.3 \% \\
-1.5 \% \\
0 \%\end{array}$ \\
\hline
\end{tabular}


Table 4. Cont.

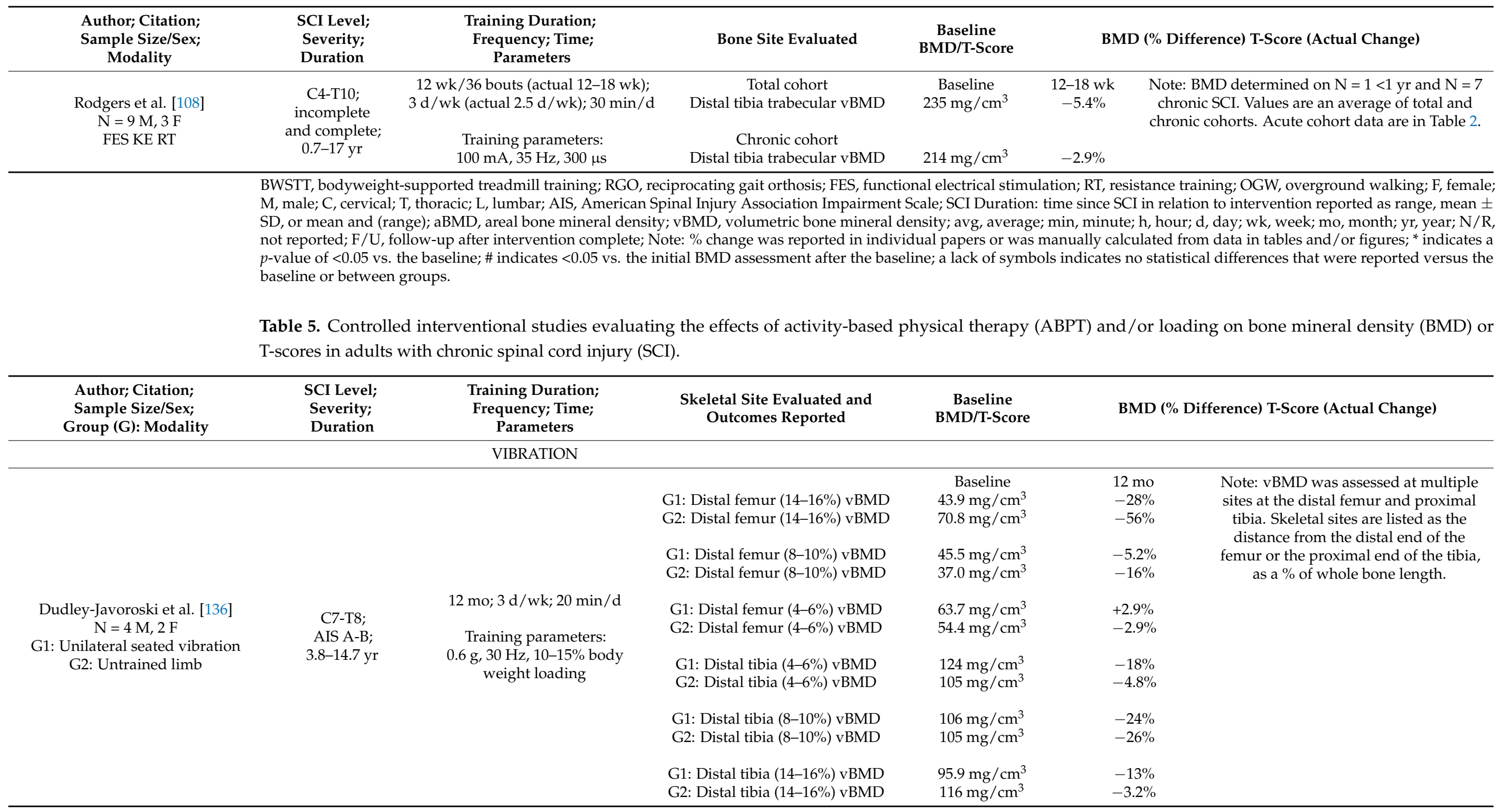


Table 5. Cont.

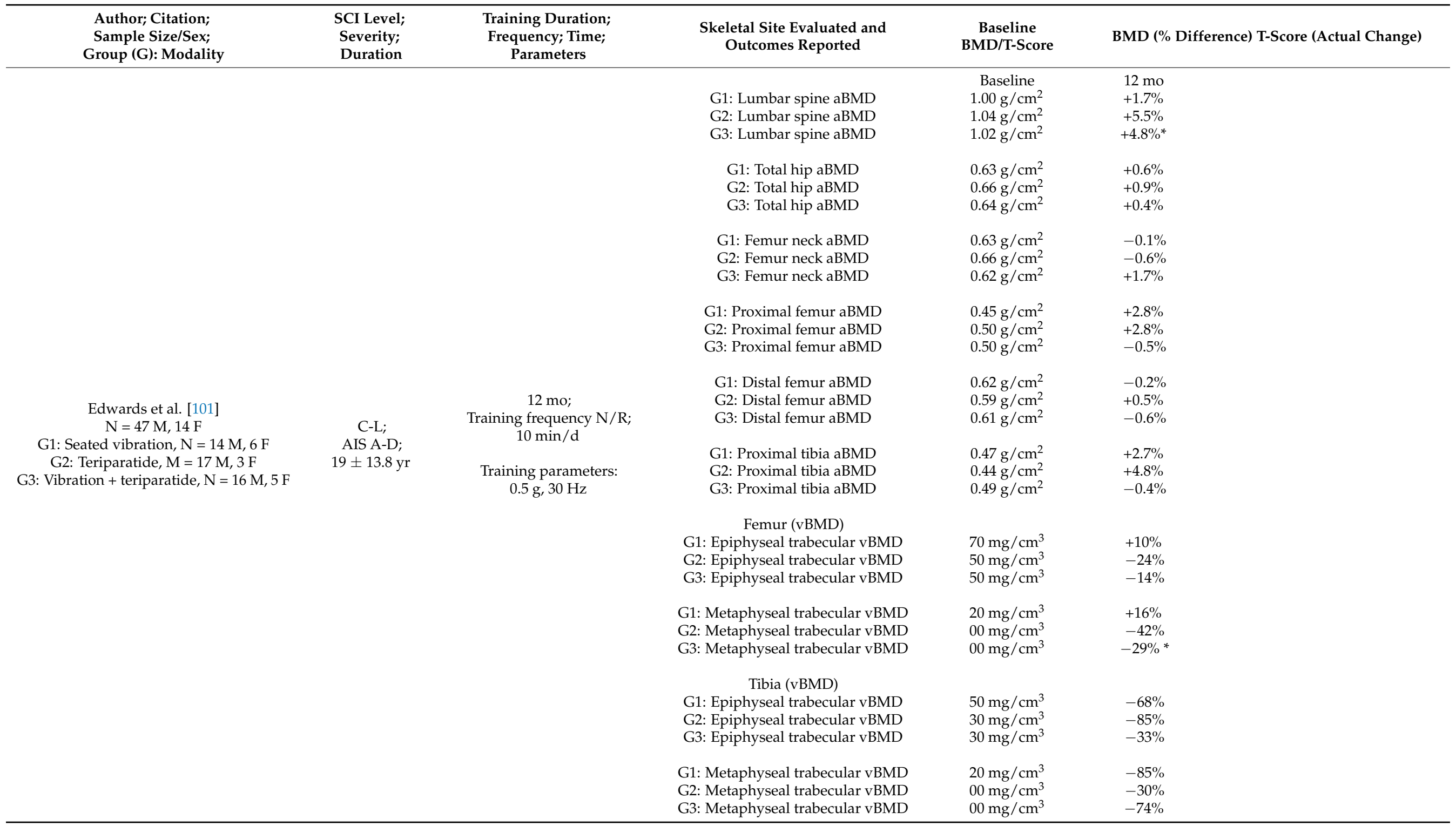


Table 5. Cont.

\begin{tabular}{|c|c|c|c|c|c|c|c|}
\hline $\begin{array}{l}\text { Author; Citation; } \\
\text { Sample Size/Sex; } \\
\text { Group (G): Modality }\end{array}$ & $\begin{array}{l}\text { SCI Level; } \\
\text { Severity; } \\
\text { Duration }\end{array}$ & $\begin{array}{l}\text { Training Duration; } \\
\text { Frequency; Time; } \\
\text { Parameters }\end{array}$ & $\begin{array}{l}\text { Skeletal Site Evaluated and } \\
\text { Outcomes Reported }\end{array}$ & $\begin{array}{c}\text { Baseline } \\
\text { BMD/T-Score }\end{array}$ & \multicolumn{3}{|c|}{ BMD (\% Difference) T-Score (Actual Change) } \\
\hline \multicolumn{8}{|c|}{ BWSTT/OGW } \\
\hline $\begin{array}{c}\text { Mobarake et al. [97] } \\
\mathrm{N}=17 \mathrm{M} \\
\text { G1: BWSTT + OGW + RT, N=10 M } \\
\text { G2: OGW + RT, N = 7 M }\end{array}$ & $\begin{array}{l}\text { Level N/R; } \\
\text { AIS B-C; } \\
\text { >6 mo }\end{array}$ & $\begin{array}{c}12 \text { wk; } 4 \mathrm{~d} / \text { wk; } 60 \mathrm{~min} / \mathrm{d} \\
\\
\text { Training parameters: } \\
\text { G1: } 0-50 \% \text { BWSTT, } \\
\text { bodyweight support reduced } \\
\text { over time, } \\
0.3 \mathrm{~km} / \mathrm{h} \\
\text { G2: OGW and RT }\end{array}$ & $\begin{array}{l}\text { G1: Femur neck aBMD } \\
\text { G2: Femur neck aBMD } \\
\text { G1: Lumbar spine aBMD } \\
\text { G2: Lumbar spine aBMD }\end{array}$ & $\begin{array}{c}\text { Baseline } \\
0.78 \mathrm{~g} / \mathrm{cm}^{2} \\
0.75 \mathrm{~g} / \mathrm{cm}^{2} \\
\\
0.89 \mathrm{~g} / \mathrm{cm}^{2} \\
0.85 \mathrm{~g} / \mathrm{cm}^{2}\end{array}$ & $\begin{array}{c}12 \mathrm{wk} \\
+17 \%++ \\
+1.3 \% \\
+9.0 \%++ \\
+1.2 \%\end{array}$ & & \\
\hline \multicolumn{8}{|c|}{ FES } \\
\hline $\begin{array}{c}\text { Belanger et al. [137] } \\
\quad \mathrm{N}=22 \mathrm{M}, 6 \mathrm{~F} \\
\text { G1: FES KE PRT limb, } \mathrm{N}=11 \mathrm{M}, 3 \mathrm{~F} \\
\text { G2: FES KE no PRT limb, } \mathrm{N}=11 \mathrm{M}, 3 \mathrm{~F} \\
\text { G3: Normative non-SCI, } \mathrm{N}=11 \mathrm{M}, 3 \mathrm{~F}\end{array}$ & $\begin{array}{l}\text { C5-T5; } \\
\text { AIS A-C; } \\
1.2-23 \mathrm{yr}\end{array}$ & $\begin{array}{c}24 \mathrm{wk} ; 5 \mathrm{~d} / \mathrm{wk} ; 1 \mathrm{~h} / \mathrm{d} \\
\text { Training parameters: } \\
\text { 10-150 mA, } 25 \mathrm{~Hz}, 300 \mu \mathrm{s}, \\
5 \mathrm{~s} \text { on:off }\end{array}$ & $\begin{array}{l}\text { G1: Distal femur aBMD } \\
\text { G2: Distal femur aBMD } \\
\text { G3: Distal femur aBMD } \\
\text { G1: Proximal tibia aBMD } \\
\text { G2: Proximal tibia aBMD } \\
\text { G3: Proximal tibia aBMD } \\
\text { G1: Tibia diaphysis aBMD } \\
\text { G2: Tibia diaphysis aBMD } \\
\text { G3: Tibia diaphysis aBMD }\end{array}$ & $\begin{array}{c}\text { Baseline } \\
\\
0.7 \mathrm{~g} / \mathrm{cm}^{2} \\
0.5 \mathrm{~g} / \mathrm{cm}^{2} \\
0.4 \mathrm{~g} / \mathrm{cm}^{2} \\
0.5 \mathrm{~g} / \mathrm{cm}^{2} \\
0.4 \mathrm{~g} / \mathrm{cm}^{2} \\
0.3 \mathrm{~g} / \mathrm{cm}^{2} \\
1.5 \mathrm{~g} / \mathrm{cm}^{2} \\
1.1 \mathrm{~g} / \mathrm{cm}^{2} \\
1.1 \mathrm{~g} / \mathrm{cm}^{2}\end{array}$ & $\begin{array}{c}24 \mathrm{wk} \\
+18 \% \text { * } \\
+18 \% \text { * } \\
\mathrm{N} / \mathrm{R} \\
+15 \% \text { * } \\
+15 \% \text { * } \\
\mathrm{N} / \mathrm{R} \\
\\
+0.0 \% \\
+0.0 \% \\
\mathrm{~N} / \mathrm{R}\end{array}$ & \multicolumn{2}{|c|}{$\begin{array}{l}\text { Note: Post-training BMD change } \\
\text { was not different between G1 and } \\
\text { G2. \% difference is an average } \\
\text { change for G1 and G2 combined. }\end{array}$} \\
\hline $\begin{array}{c}\text { Bloomfield et al. [96] } \\
\mathrm{N}=10 \mathrm{M}, 7 \mathrm{~F} \\
\text { G1: FES KE RT + cycling, } \mathrm{N}=5 \mathrm{M}, 4 \mathrm{~F} \\
\text { G2: SCI controls, } \mathrm{N}=5 \mathrm{M}, 3 \mathrm{~F}\end{array}$ & $\begin{array}{c}\text { G1: C5-T7; Frankel } \\
\text { A-B; } \\
\quad 6 \pm 1.2 \mathrm{yr} \\
\text { G2: C4-T12; Frankel } \\
\text { A-B; } 8.3 \pm 2.3 \mathrm{yr}\end{array}$ & $\begin{array}{c}5.9 \pm 1.0 \text { wk FES KE RT } \\
6 \text { mo FES cycling ( } 80 \text { sessions); } \\
3 \mathrm{~d} / \mathrm{wk} ; 30 \mathrm{~min} / \mathrm{d} \\
\text { Training parameters: } \\
0-130 \mathrm{~mA}, 30 \mathrm{~Hz}, 350 \mathrm{~ms} \\
50 \mathrm{rpm}\end{array}$ & $\begin{array}{l}\text { G1: Lumbar spine aBMD } \\
\text { G2: Lumbar spine aBMD } \\
\text { G1: Femur neck aBMD } \\
\text { G2: Femur neck aBMD } \\
\text { G1: Distal femur aBMD } \\
\text { G2: Distal femur aBMD } \\
\text { G1: Proximal tibia aBMD } \\
\text { G2: Proximal tibia aBMD }\end{array}$ & $\begin{array}{c}\text { Baseline } \\
1.23 \mathrm{~g} / \mathrm{cm}^{2} \\
1.28 \mathrm{~g} / \mathrm{cm}^{2} \\
\\
0.80 \mathrm{~g} / \mathrm{cm}^{2} \\
0.70 \mathrm{~g} / \mathrm{cm}^{2} \\
\\
0.47 \mathrm{~g} / \mathrm{cm}^{2} \\
0.52 \mathrm{~g} / \mathrm{cm}^{2} \\
\\
0.36 \mathrm{~g} / \mathrm{cm}^{2} \\
0.39 \mathrm{~g} / \mathrm{cm}^{2}\end{array}$ & $\begin{array}{c}3 \mathrm{mo} \\
+1.6 \% \\
+2.4 \% \\
-2.1 \% \\
-1.9 \% \\
\\
+6.8 \% \\
+2.1 \% \\
\\
-2.8 \% \\
-0.3 \%\end{array}$ & $\begin{array}{l}6 \mathrm{mo} \\
+1.4 \% \\
+0.4 \% \\
\\
-2.0 \% \\
-1.7 \% \\
\\
+4.9 \% \\
-2.3 \% \\
-5.3 \% \\
-3.9 \%\end{array}$ & $\begin{array}{c}9 \mathrm{mo} \\
+3.8 \% \text { * } \\
\mathrm{N} / \mathrm{R} \\
\\
-3.9 \% \\
\mathrm{~N} / \mathrm{R} \\
\\
+10 \% \\
\mathrm{~N} / \mathrm{R} \\
\\
+4.2 \% \\
\mathrm{~N} / \mathrm{R}\end{array}$ \\
\hline
\end{tabular}


Table 5. Cont.

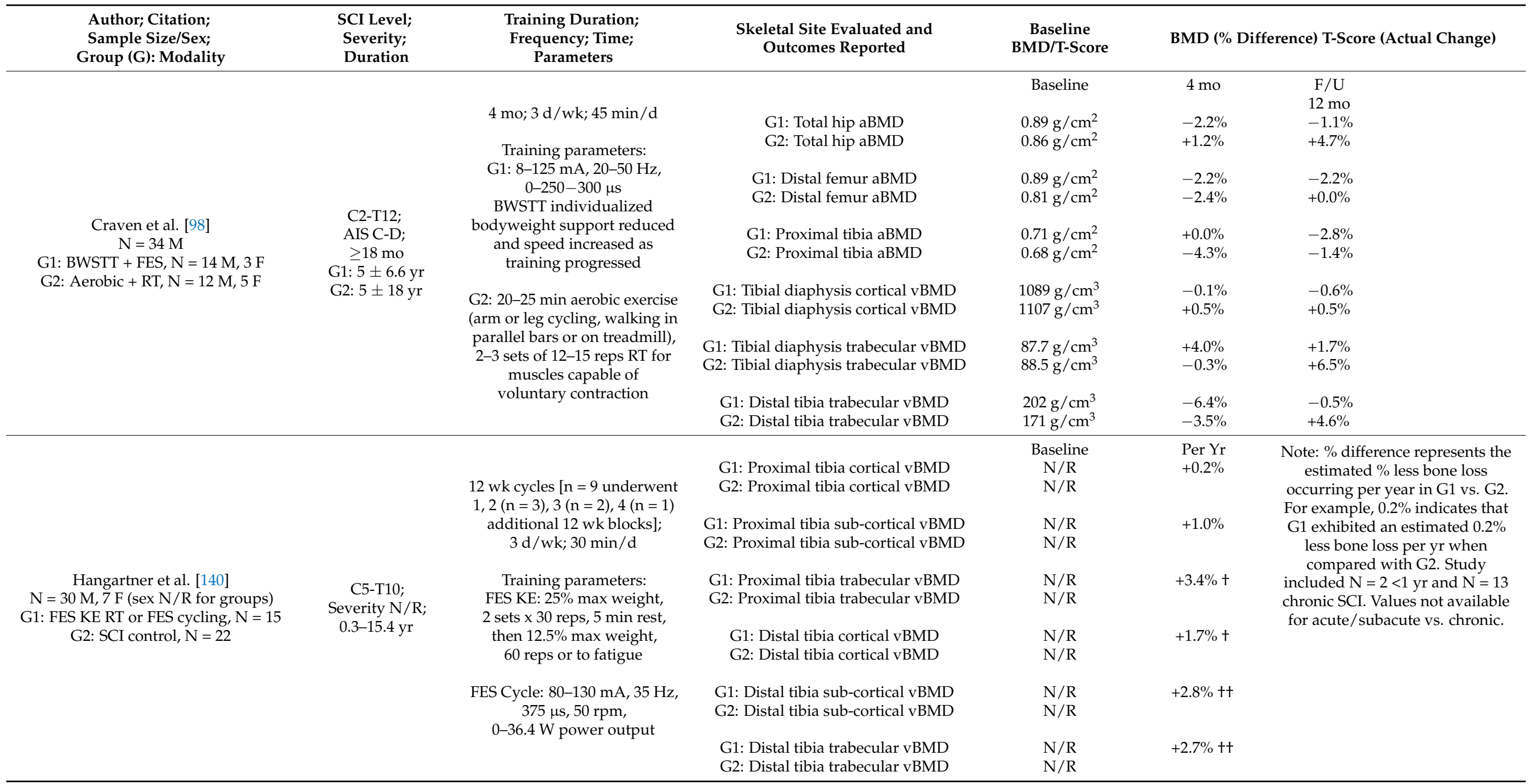


Table 5. Cont.

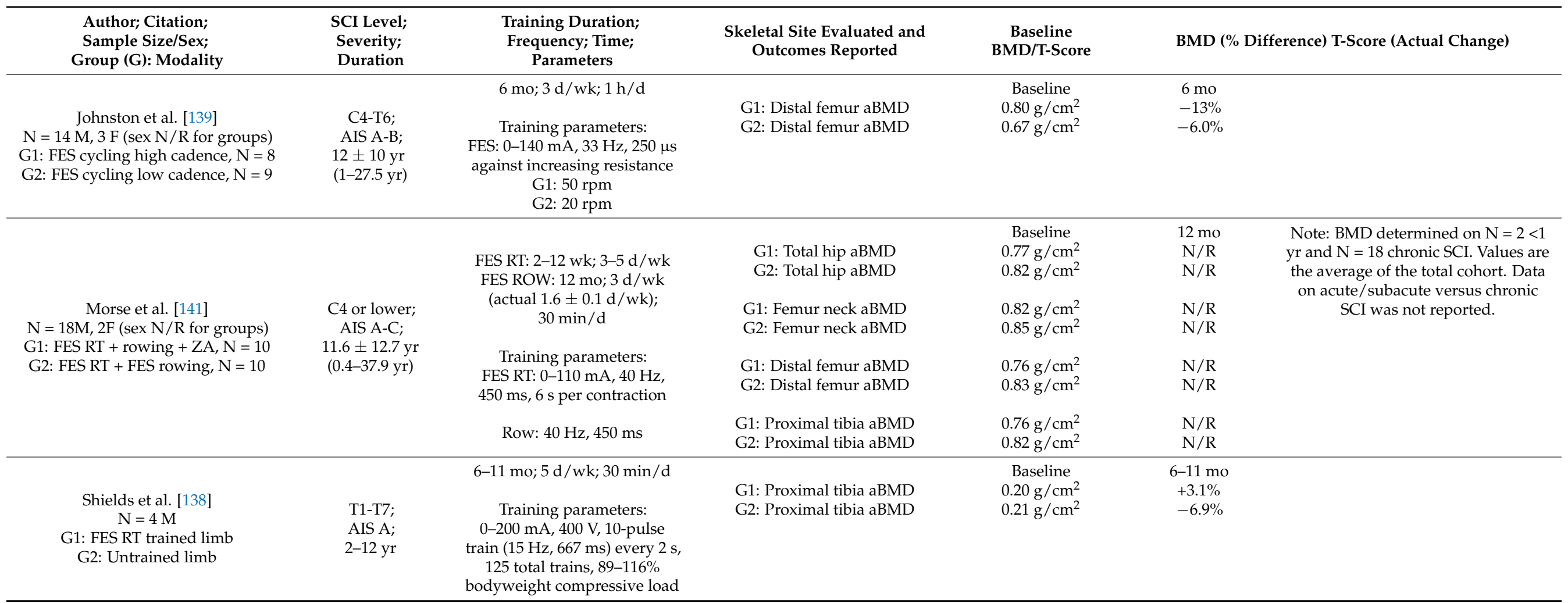

G, group; BWSTT, bodyweight-supported treadmill training; FES, functional electrical stimulation; RT, resistance training; PRT, progressive resistance training; OGW, overground walking; ZA, zoledronic acid; F, female; M, male; C, cervical; T, thoracic; L, lumbar; AIS, American Spinal Injury Association Impairment Scale; SCI Duration: time since SCI in relation to intervention reported as range, mean $\pm \mathrm{SD}$, or mean and (range); aBMD, areal bone mineral density; vBMD, volumetric bone mineral density; min, minute; h, hour; d, day; wk, week; mo, month; yr, year; N/R, not reported; Note: \% change was reported in individual papers or was manually calculated from data in tables and/or figures; * indicates a $p$-value of $<0.05$ vs. the baseline; $\uparrow$ indicates $<0.05,+\uparrow<0.01$ between the groups; a lack of symbols indicates no statistical differences were reported versus the baseline or between the groups 
In total, five uncontrolled [13,29,30,100,103] and nine controlled trials [96,98,101,136-141] evaluated distal femur and/or proximal tibia BMD. Most of these studies reported neither an attenuation of BMD loss nor increased BMD at areas surrounding the knee [96,98,100,101,103, $136,138,139,141]$. However, a small subset of these trials reported improved BMD at the knee, with each study using FES-based RT and/or cycling [29,30,134,137,140]. For example, the distal femur and/or proximal tibia aBMD increased 10-15\% vs. the baseline in studies that utilized 6-18 months of FES knee extension RT (distal femur: $+18 \%$, proximal tibia: $+15 \%$ ) [137] or cycling (distal femur: $+11 \%$, proximal tibia: $+10-13 \%$ ) $[29,30]$. Similarly, the total and trabecular vBMD at the distal femur were improved $7 \%$ to $13 \%$ vs. the baseline, respectively, in a study that utilized three-months of FES knee extension RT followed by nine-months of FES cycling [134], suggesting that trabecular bone responds more robustly to FES than cortical bone. In this regard, Hangartner et al. reported that 12-weeks of FES knee extension RT followed by 12- to 36-weeks of FES cycling produced an estimated 3.3\% less proximal tibia trabecular vBMD loss per year when compared with non-exercised controls, while no apparent cortical vBMD improvement existed [140]. A total of two trials that incorporated bodyweight-supported [97] or full weight-bearing activity without FES [103] also observed an increased aBMD at sites other than the knee. Mobarake et al. [97] reported that a 12-week BWSTT regimen increased the lumbar spine and femoral neck $a B M D$ by $+9 \%$ and $+17 \%$ from the baseline, respectively, vs. a $+1.2-1.3 \%$ change for the controls. Similarly, Astorino et al. [103] reported that a six-month multimodal ABPT regimen increased lumbar spine aBMD by $+4.7 \%$ vs. the baseline. However, as indicated above, seven trials that assessed FES-based modalities $[108,132,133,135,138,139,141]$ and several that incorporated standing without FES [102,128] or exoskeleton/assisted walking/BWSTT without FES $[100,129,130]$ reported no BMD improvement, irrespective of the site that was examined.

\section{Common Parameters to Improve BMD after SCI}

Several common parameters existed among studies that reported improved proximal tibia or distal femur BMD or increased BMD at other sites. First, studies that reported attenuated BMD loss at the knee all enrolled persons with acute/subacute SCI and used FES-based modalities that were performed 3-5 days/week, 20-60 min/day, for $\geq 3$-months $[28,31,106,113]$ and studies reporting increased BMD at the knee all enrolled persons with chronic SCI and used FES-based regimens performed 3-5 days/week, $30+\mathrm{min} /$ day, for $\geq 6$-months $[29,30,134,137]$. Second, no study that incorporated standing without FES observed improvements in the distal femur or proximal tibia BMD, although, some that utilized these regimens 3-7 days/week, 60+ min/day, for $>3-$ months reported attenuated BMD loss [107,109,112] or increased BMD at other sites [97,103]. These observations highlight the need to target ABPTs to the specific region(s) where BMD improvements are most warranted and to use a training intensity, frequency, and duration that is sufficient to improve BMD. Regardless, it is important to reiterate that not all FES modalities or passive/active standing regimens that met these criteria produced BMD improvements. For example, Clark et al. [111] and Arija-Blazquez et al. [99] reported that FES-based RT regimens lasting 14-weeks to 5-months (performed 5-days/week for 30+ min/day) did not attenuate sublesional aBMD loss in persons with acute/subacute SCI and other FES studies that met the chronic criteria reported no BMD improvement at the distal femur or proximal tibia $[96,138,139]$, or at other skeletal sites [108,131,135].

Several other factors that were associated with BMD improvements were also identified. First, higher total work output was associated with greater BMD gain. For example, Lambach et al. [106] reported that a higher magnitude of loading and more weekly training sessions were both associated with less trabecular vBMD loss at the proximal femur in persons that were undergoing FES rowing, and Draghici et al. [142] reported that total distance rowed and peak foot reaction force were positively associated with the preservation of the distal tibia trabecular thickness in persons that were undergoing FES rowing, when these factors and SCI duration were incorporated into stepwise regression models. 
Similarly, Bloomfield et al. [96] reported that distal femur aBMD was improved (+17.8\% vs. the baseline) in a subgroup of persons who achieved power outputs $>18$ watts (W) during FES cycling, while aBMD was not improved in those with lower power output $(<12 \mathrm{~W}$, BMD change $+0.2 \%)$ or in non-exercising controls $(-2.3 \%$ vs. the baseline) and Frotzler et al. [134] reported that FES cycling improved the total and trabecular vBMD at the distal femur with participants averaging $\sim 18 \mathrm{~W}$ power output. Regardless, $18 \mathrm{~W}$ may be difficult to sustain for many persons with SCI. As evidence, Eser et al. [143] reported that power outputs ranged from 10.8 to $13.6 \mathrm{~W}$ in persons with acute SCI who completed an average of 47 FES cycling bouts, with higher FES frequency producing higher power outputs. Of these participants, less than one-third achieved an average power output $>18 \mathrm{~W}$. Second, a continued training stimulus is required to maintain BMD if skeletal improvements are achieved. As evidence, in two studies that reported FES increased aBMD vs. the baseline, a regression of BMD to the baseline occurred after a six-month period involving no FES cycling [29] or once weekly FES [30].

\section{Future Directions}

As discussed above, only limited evidence supports the notion that ABPTs attenuate BMD loss in persons with acute/subacute SCI or increase BMD in the paralyzed limbs after chronic SCI, especially when considering the most fracture-prone sites that surround the knee. Moreover, few studies have evaluated whether ABPTs improve trabecular or cortical bone microstructure at the distal femur or proximal tibia (Table 6) and none have assessed whether the potential BMD gains or bone microstructural changes mitigate the fracture risk. Interestingly, Fang et al. [144] and Edwards et al. [101] both used FEA to predict the bone mechanical characteristics in response to 12-months of FES rowing or seated vibration, respectively, and observed no tensile or compressive strength improvements at the distal femur or proximal tibia. However, neither study observed BMD or bone microstructural changes at areas surrounding the knee in response to the respective modalities. Given these observations, we suggest future research focuses on optimizing the FES parameters to improve the distal femur and proximal tibia bone parameters, with emphasis on the following questions:

- What are the optimal FES and modality-specific parameters (e.g., stimulation frequency, amplitude, pulse width, power output, tibio-femoral strain, and pedaling cadence) to stimulate and/or maintain bone improvements?

- What is the optimal training frequency (number of sessions per day or per week) and training duration (time per bout and intervention length) to improve bone parameters?

- What is the minimum training frequency and duration to maintain bone after SCI?

- Is the magnitude of BMD or bone microstructural gains that result from FES sufficient to improve bone strength or bone mechanical characteristics and/or to reduce the fracture risk at the distal femur and/or proximal tibia after SCI? 
Table 6. Interventional studies evaluating the effects of activity-based physical therapy (ABPT) and/or loading on bone microstructural in adults with subacute and/or chronic spinal cord injury (SCI).

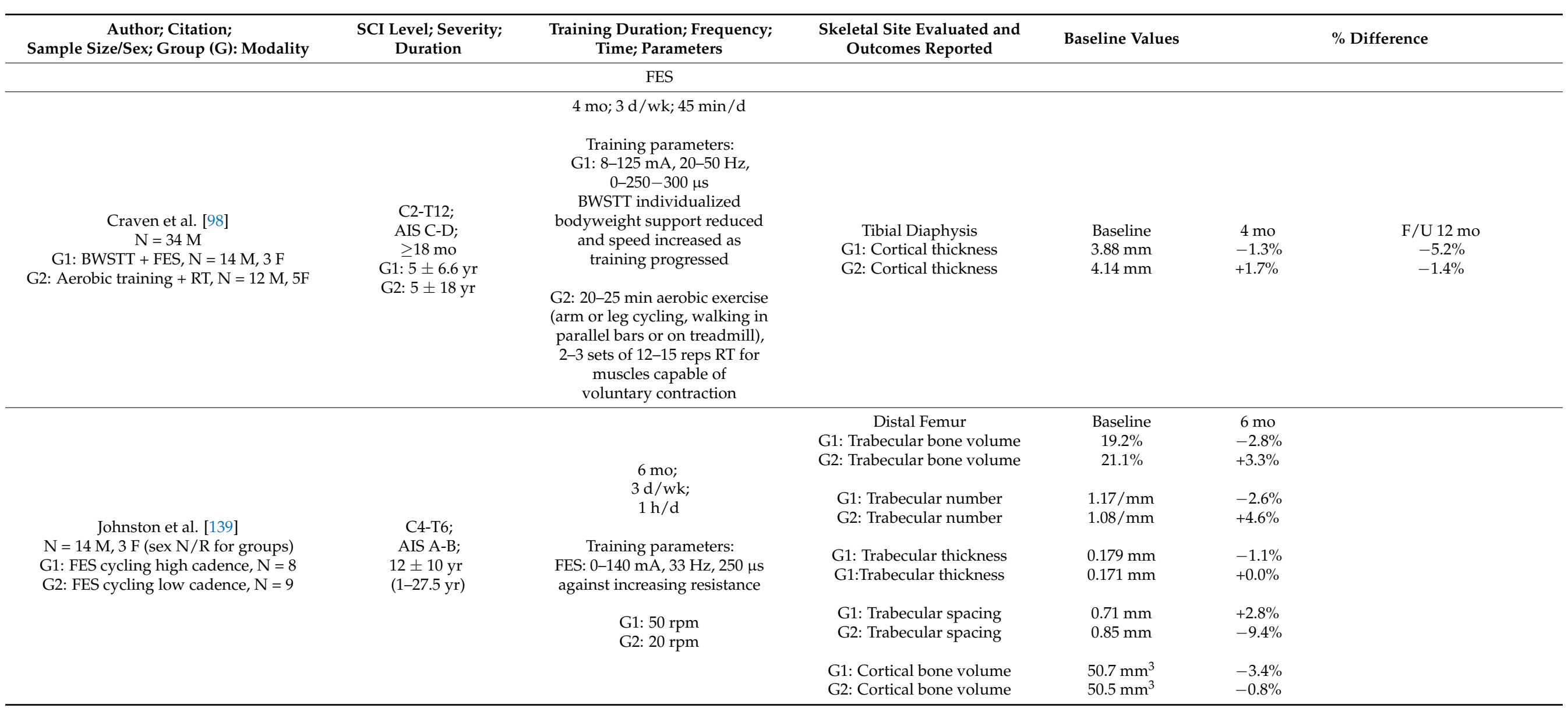


Table 6. Cont.

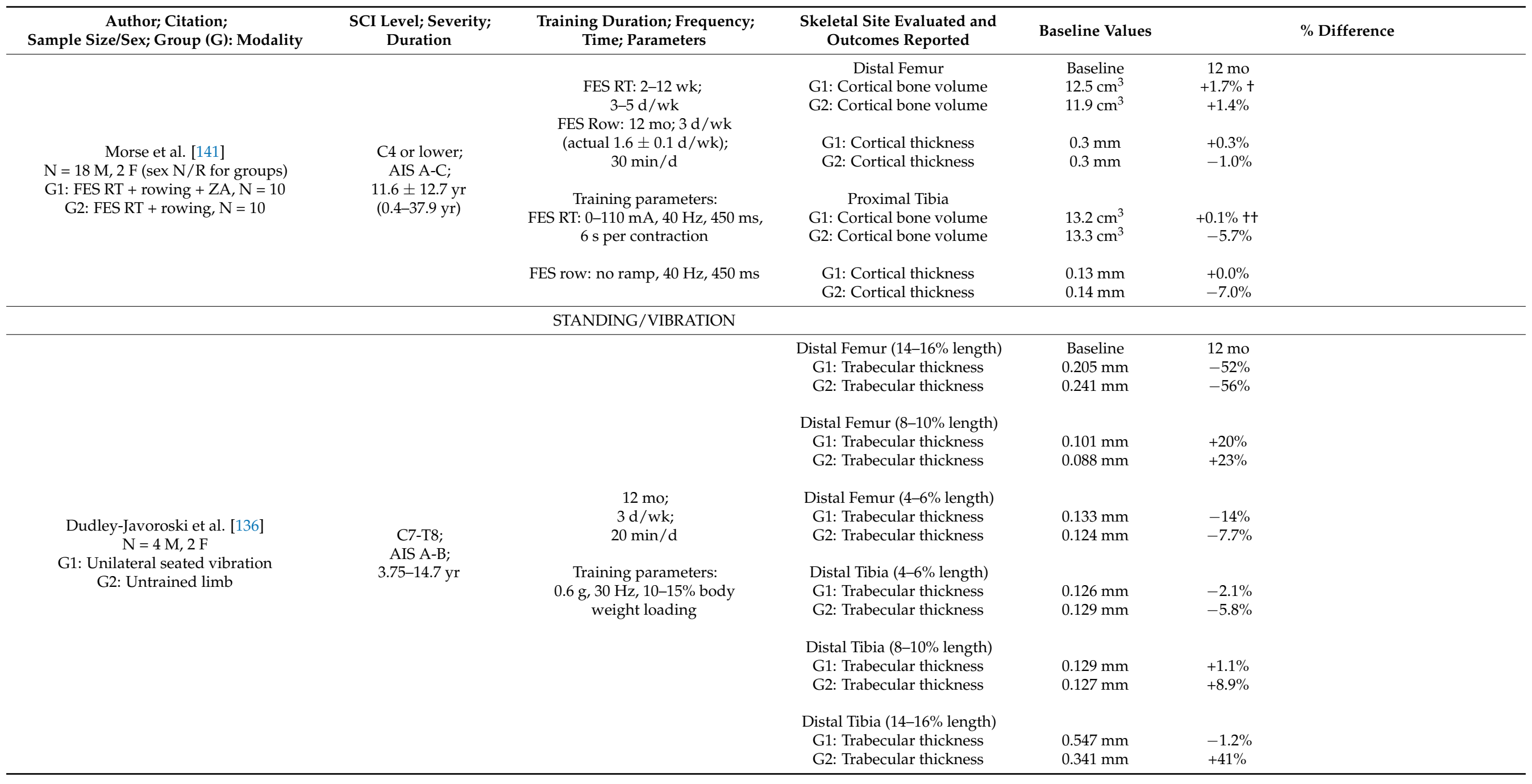


Table 6. Cont.

\begin{tabular}{|c|c|c|c|c|c|c|c|}
\hline $\begin{array}{c}\text { Author; Citation; } \\
\text { Sample Size/Sex; Group (G): Modality }\end{array}$ & $\begin{array}{l}\text { SCI Level; Severity; } \\
\text { Duration }\end{array}$ & $\begin{array}{l}\text { Training Duration; Frequency; } \\
\text { Time; Parameters }\end{array}$ & $\begin{array}{l}\text { Skeletal Site Evaluated and } \\
\text { Outcomes Reported }\end{array}$ & Baseline Values & & \% Difference & \\
\hline \multirow{22}{*}{$\begin{array}{c}\text { Edwards et al. [101] } \\
\mathrm{N}=47 \mathrm{M}, 14 \mathrm{~F} \\
\text { G1: Seated vibration, } \mathrm{N}=14 \mathrm{M}, 6 \mathrm{~F} \\
\text { G2: TA, } \mathrm{M}=17 \mathrm{M}, 3 \mathrm{~F} \\
\text { G3: Seated vibration + TA, N = 16 M, } 5 \mathrm{~F}\end{array}$} & \multirow{22}{*}{$\begin{array}{l}\text { C-L; } \\
\text { AIS A-D; } \\
19 \pm 13.8 \mathrm{yr}\end{array}$} & \multirow{12}{*}{$\begin{array}{l}12 \mathrm{mo} \\
\text { Frequency N/R; } \\
10 \mathrm{~min} / \mathrm{d}\end{array}$} & Femur Diaphysis & Baseline & $12 \mathrm{mo}$ & & \\
\hline & & & G1: Cortical bone volume & $13.0 \mathrm{~cm}^{3}$ & $-0.1 \%$ & & \\
\hline & & & G2: Cortical bone volume & $13.9 \mathrm{~cm}^{3}$ & $+0.2 \%$ & & \\
\hline & & & Distal Femur Metaphysis & & & & \\
\hline & & & G1: Cortical bone volume & $10.4 \mathrm{~cm}^{3}$ & $+1.1 \%$ & & \\
\hline & & & G2: Cortical bone volume & $11.5 \mathrm{~cm}^{3}$ & $+3.8 \%$ & & \\
\hline & & & G3: Cortical bone volume & $11.3 \mathrm{~cm}^{3}$ & $+1.7 \%$ & & \\
\hline & & & Distal Femur Epiphysis & & & & \\
\hline & & & G1: Cortical bone volume & $2.95 \mathrm{~cm}^{3}$ & $+3.8 \%$ & & \\
\hline & & & G2: Cortical bone volume & $2.43 \mathrm{~cm}^{3}$ & $+11 \%$ & & \\
\hline & & & G3: Cortical bone volume & $2.52 \mathrm{~cm}^{3}$ & $+1.3 \%$ & & \\
\hline & & & & & & & \\
\hline & & \multirow{6}{*}{$\begin{array}{l}\text { Training parameters: } \\
0.5 \mathrm{~g}, 30 \mathrm{~Hz}\end{array}$} & Proximal Tibia Epiphysis & & & & \\
\hline & & & G1: Cortical bone volume & $2.64 \mathrm{~cm}^{3}$ & $+10 \%$ & & \\
\hline & & & G2: Cortical bone volume & $2.79 \mathrm{~cm}^{3}$ & $+14 \%$ & & \\
\hline & & & G3: Cortical bone volume & $3.17 \mathrm{~cm}^{3}$ & $+7.1 \%$ & & \\
\hline & & & G2: Cortical bone volume & $11.9 \mathrm{~cm}^{3}$ & $+1.7 \%$ & & \\
\hline & & & G3: Cortical bone volume & $11.9 \mathrm{~cm}^{3}$ & $+2.1 \%$ & & \\
\hline & & & Tibia Diaphysis & & & & \\
\hline & & & G1: Cortical bone volume & $13.2 \mathrm{~cm}^{3}$ & $+22 \%$ & & \\
\hline & & & G2: Cortical bone volume & $14.7 \mathrm{~cm}^{3}$ & $+0.8 \%$ & & \\
\hline & & & G3: Cortical bone volume & $14.0 \mathrm{~cm}^{3}$ & $+0.4 \%$ & & \\
\hline \multirow{6}{*}{$\begin{array}{l}\text { Wuermser et al. [102] } \\
\quad \mathrm{N}=5 \mathrm{M}, 4 \mathrm{~F} \\
\text { Standing + vibration }\end{array}$} & \multirow{6}{*}{$\begin{array}{l}\text { AIS A-B; } \\
\text { T3-T12; } \\
\text { 2-27 yr }\end{array}$} & $6 \mathrm{mo} ; 5 \mathrm{~d} / \mathrm{wk} ; 20 \mathrm{~min} / \mathrm{d}$ & Distal Tibia & Baseline & $\begin{array}{l}3 \mathrm{mo} \\
+0.9 \%\end{array}$ & $\begin{array}{l}6 \mathrm{mo} \\
-5.5 \%\end{array}$ & $\begin{array}{l}\mathrm{F} / \mathrm{U} \\
6 \mathrm{mo}\end{array}$ \\
\hline & & \multirow{5}{*}{$\begin{array}{c}\text { Training parameters: } \\
0.3 \mathrm{~g}, 34 \mathrm{~Hz} \text { sinusoidal movement } \\
\text { of } 50 \mu \mathrm{m} \text { w / lower extremities } \\
\text { supporting } \sim 86 \% \text { body weight }\end{array}$} & Trabecular number & $1.09 / \mathrm{mm}$ & $+0.0 \%$ & $+0.0 \%$ & $-7.3 \%$ \\
\hline & & & Trabecular thickness & $0.04 \mathrm{~mm}$ & $+8.7 \%$ & $+7.6 \%$ & $+0.0 \%$ \\
\hline & & & Trabecular separation & $1.15 \mathrm{~mm}$ & $-2.5 \%$ & $-1.3 \%$ & $+15 \%$ \\
\hline & & & Cortical thickness & $0.80 \mathrm{~mm}$ & $-3.3 \%$ & $-1.8 \%$ & $-3.8 \%$ \\
\hline & & & Cortical area & $86.5 \mathrm{~mm}^{2}$ & & & $-3.5 \%$ \\
\hline
\end{tabular}

G, group; BWSTT, bodyweight supported treadmill training; FES, functional electrical stimulation; RT, resistance training; ZA, zoledronic acid; TA, teriparatide F, female; M, male; C, cervical; T, thoracic; L, lumbar; AIS, American Spinal Injury Association Impairment Scale. SCI Duration: time since SCI in relation to intervention reported as range, $\mathrm{mean} \pm \mathrm{SD}, \mathrm{O}$

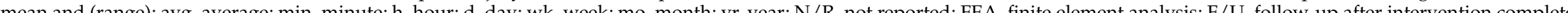
Note: \% cos indicates no statistical differences that were reported versus the baseline or between the groups. 
Clinical trials focused on optimizing FES would require a large, concerted effort over many years to discern optimal parameters, due to the relatively slow bone remodeling in humans and the number of potential parameter combinations. To lessen this burden, percutaneous ES [105,145,146] or direct nerve stimulation [64,104,147] techniques could be used to optimize the FES parameters and to identify the optimal training frequency and duration in preclinical SCI models, with only the most successful protocols advancing. Preclinical SCI models can also be used to evaluate combinatory strategies to improve BMD. In this regard, several recent preclinical and clinical studies have assessed ABPTs combined with antiresorptive drugs, such as zoledronic acid [141,144] or testosterone [59,104,148], or bone anabolic drugs such as teriparatide [101], with varying success.

Regardless of the intervention that is used, we suggest the following practices be implemented when evaluating bone changes after SCI. First, studies should quantify the distal femur and/or proximal tibia bone parameters before and after an intervention using an established imaging modality (e.g., DXA or pQCT) and a validated protocol, instead of assessing surrogate bone sites. We recognize that DXA is used to quantify whole-body fat-free mass or fat mass changes, and thus, aBMD that is assessed in the whole-body or at other traditional osteoporosis sites may be reported out of convenience. Future work should explain the relevance of BMD changes at these traditional osteoporosis sites in relation to the more fracture-prone distal femur and proximal tibia sites after SCI. Second, clinical trials should last a minimum of 6-12 months with ABPT performed $\geq 3$-days/week for $\geq 30 \mathrm{~min} /$ day across the intervention, given the slow bone turnover and BMD improvements that are present in humans in response to loading. Third, a control group or control comparator (e.g., untrained limb) should be assessed to ensure the appropriate data interpretation. Fourth, interventions should attempt to quantify the tibio-femoral forces during training, as has been previously reported [149], and/or attempt to achieve average power outputs $>18 \mathrm{~W}$ when utilizing FES cycling as regimens exceeding this threshold appear to have a higher likelihood to improve BMD [96]. Fifth, studies should assess the fracture risk and/or evaluate the bone tensile properties via FEA or another method, if available, to establish ABPT effectiveness because the reduction in bone strength over the first few months after SCI has been estimated to be three times greater than the aBMD loss as determined by DXA [36] and because any given percentage increase in whole-bone BMD resulting from loading has been associated with 10 times greater percentage increase in bone strength in preclinical models [90], suggesting that BMD changes do not reflect the bone mechanical properties that may influence non-traumatic fracture risk.

\section{Conclusions}

A visual summary of all the reviewed studies examining the effects of ABPT modalities at knee and non-knee sites in acute and chronic SCI is presented in Figure 3A,B. Various ABPTs promote neuromuscular benefits and use-dependent neuroplasticity in persons with SCI [78]. Despite this, no known ABPT completely prevents bone loss that develops in the lower extremities over the acute/subacute post-SCI period, regardless of the skeletal site that is evaluated. Moreover, no ABPT has shown universal success in increasing BMD at the highly fracture-prone sites surrounding the knee. However, a small subset of trials that evaluated FES modalities reported attenuated BMD loss at the distal femur and/or proximal tibia in persons with acute to subacute SCI and increased BMD in those with chronic SCI, suggesting such regimens hold promise. Additionally, a small subset of studies that utilized weight-bearing ABPTs without FES reported BMD gains at other sites but no BMD changes in areas near the knee. As such, research is needed to understand why ABPTs produce variable BMD responses after SCI and to optimize the training parameters for bone gain. Moreover, routine monitoring of the distal femur and proximal tibia BMD appears important to assess the fracture risk before engaging in ABPTs and to monitor bone changes at these highly fracture-prone sites. 


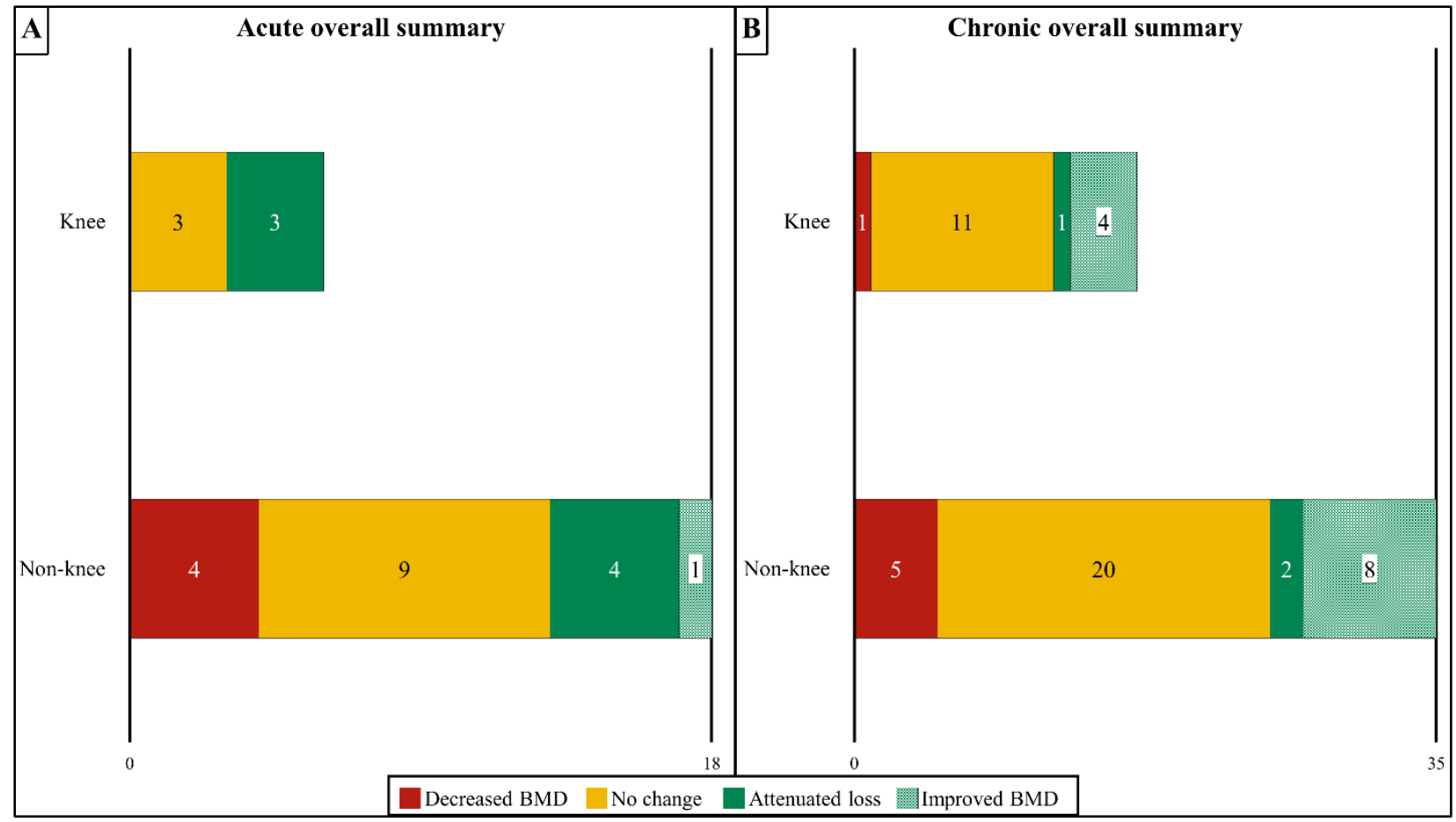

Figure 3. Summary of the effects of activity-based physical therapy (ABPT) on changes in bone mineral density (BMD) at the knee (distal femur and proximal tibia) or all other sublesional non-knee sites for persons with acute/sub-acute (A) or chronic (B) SCI in aggregate. Overall, half of all acute studies and most chronic studies showed no effect on BMD at the knee or non-knee sites. For data on specific ABPT modalities see Figure 1A,B (acute/subacute SCI) or Figure 2A,B (chronic SCI).

Author Contributions: Conceptualization, T.W.S., J.K. and J.F.Y.; methodology, T.W.S., D.M.O. and J.F.Y.; formal analysis, T.W.S., J.K., A.J.M., D.M.O. and J.F.Y.; writing-original draft preparation, T.W.S.; writing—review and editing, J.K., A.J.M., D.M.O. and J.F.Y.; visualization, T.W.S., J.K. and J.F.Y.; supervision, J.F.Y.; project administration, J.F.Y.; funding acquisition, J.F.Y. All authors have read and agreed to the published version of the manuscript.

Funding: This research was funded by the Department of Veterans Affairs Office of Research and Development, Rehabilitation Research and Development Service Merit Award \#1I01RX002447-01 and PECASE \#B9280-O to J.F.Y. and by resources provided by the North Florida/South Georgia Veterans Health System. The work reported herein does not represent the views of the US Department of Veterans Affairs or the US Government.

Institutional Review Board Statement: Not applicable.

Informed Consent Statement: Not applicable.

Data Availability Statement: The data presented in this study are available on request from the corresponding author. The data are not publicly available due to data being property of the United States federal government.

Conflicts of Interest: The authors declare no conflict of interest.

\section{References}

1. WHO. Spinal Cord Injury Fact Sheets; World Health Organization: Geneva, Switzerland, 2013; Available online: http:/ /www.who. int/en/news-room/fact-sheets/detail/spinal-cord-injury (accessed on 12 March 2021).

2. NSCISC. National Spinal Cord Injury Statistical Center, Facts and Figures at a Glance. University of Alabama at Birmingham. 2018. Available online: https:/ / www.nscisc.uab.edu/Public/Facts\%20and\%20Figures\%20-\%202018.pdf (accessed on 8 June 2018).

3. Sezer, N.; Akkus, S.; Ugurlu, F.G. Chronic complications of spinal cord injury. World J. Orthop. 2015, 6, 24-33. [CrossRef]

4. Yarrow, J.F.; Cardozo, C.P. Effects of Spinal Cord and Related Conditions. In Encyclopedia of Bone Biology; Zaidi, M., Ed.; Elsevier: Amsterdam, The Netherlands, 2020; pp. 429-488. 
5. Morse, L.R.; Battaglino, R.A.; Stolzmann, K.L.; Hallett, L.D.; Waddimba, A.; Gagnon, D.; Lazzari, A.A.; Garshick, E. Osteoporotic fractures and hospitalization risk in chronic spinal cord injury. Osteoporos. Int. 2009, 20, 385-392. [CrossRef]

6. Kostovski, E.; Hjeltnes, N.; Eriksen, E.F.; Kolset, S.O.; Iversen, P.O. Differences in bone mineral density, markers of bone turnover and extracellular matrix and daily life muscular activity among patients with recent motor-incomplete versus motor-complete spinal cord injury. Calcif. Tissue Int. 2015, 96, 145-154. [CrossRef]

7. Grassner, L.; Klein, B.; Maier, D.; Buhren, V.; Vogel, M. Lower extremity fractures in patients with spinal cord injury Characteristics, outcome and risk factors for non-unions. J. Spinal Cord Med. 2018, 41, 676-683. [CrossRef] [PubMed]

8. Gifre, L.; Vidal, J.; Carrasco, J.; Portell, E.; Puig, J.; Monegal, A.; Guanabens, N.; Peris, P. Incidence of skeletal fractures after traumatic spinal cord injury: A 10-year follow-up study. Clin. Rehabil. 2014, 28, 361-369. [CrossRef] [PubMed]

9. Garland, D.E.; Adkins, R.H.; Kushwaha, V.; Stewart, C. Risk factors for osteoporosis at the knee in the spinal cord injury population. J. Spinal Cord Med. 2004, 27, 202-206. [CrossRef] [PubMed]

10. Zehnder, Y.; Luthi, M.; Michel, D.; Knecht, H.; Perrelet, R.; Neto, I.; Kraenzlin, M.; Zach, G.; Lippuner, K. Long-term changes in bone metabolism, bone mineral density, quantitative ultrasound parameters, and fracture incidence after spinal cord injury: A cross-sectional observational study in 100 paraplegic men. Osteoporos. Int. 2004, 15, 180-189. [CrossRef]

11. Eser, P.; Frotzler, A.; Zehnder, Y.; Wick, L.; Knecht, H.; Denoth, J.; Schiessl, H. Relationship between the duration of paralysis and bone structure: A pQCT study of spinal cord injured individuals. Bone 2004, 34, 869-880. [CrossRef]

12. Dauty, M.; Perrouin Verbe, B.; Maugars, Y.; Dubois, C.; Mathe, J.F. Supralesional and sublesional bone mineral density in spinal cord-injured patients. Bone 2000, 27, 305-309. [CrossRef]

13. Frotzler, A.; Berger, M.; Knecht, H.; Eser, P. Bone steady-state is established at reduced bone strength after spinal cord injury: A longitudinal study using peripheral quantitative computed tomography (pQCT). Bone 2008, 43, 549-555. [CrossRef]

14. Edwards, W.B.; Simonian, N.; Troy, K.L.; Schnitzer, T.J. Reduction in Torsional Stiffness and Strength at the Proximal Tibia as a Function of Time since Spinal Cord Injury. J. Bone Miner. Res. 2015, 30, 1422-1430. [CrossRef]

15. Gibbons, R.S.; Beaupre, G.S.; Kazakia, G.J. FES-rowing attenuates bone loss following spinal cord injury as assessed by HR-pQCT. Spinal Cord Ser. Cases 2016, 2, 15041. [CrossRef]

16. Frisbie, J.H. Fractures after myelopathy: The risk quantified. J. Spinal Cord Med. 1997, 20, 66-69. [CrossRef] [PubMed]

17. Cirnigliaro, C.M.; Myslinski, M.J.; La Fountaine, M.F.; Kirshblum, S.C.; Forrest, G.F.; Bauman, W.A. Bone loss at the distal femur and proximal tibia in persons with spinal cord injury: Imaging approaches, risk of fracture, and potential treatment options. Osteoporos. Int. 2017, 28, 747-765. [CrossRef] [PubMed]

18. Frotzler, A.; Cheikh-Sarraf, B.; Pourtehrani, M.; Krebs, J.; Lippuner, K. Long-bone fractures in persons with spinal cord injury. Spinal Cord 2015, 53, 701-704. [CrossRef] [PubMed]

19. Carbone, L.D.; Chin, A.S.; Burns, S.P.; Svircev, J.N.; Hoenig, H.; Heggeness, M.; Weaver, F. Morbidity following lower extremity fractures in men with spinal cord injury. Osteoporos. Int. 2013, 24, 2261-2267. [CrossRef]

20. Carbone, L.D.; Chin, A.S.; Burns, S.P.; Svircev, J.N.; Hoenig, H.; Heggeness, M.; Bailey, L.; Weaver, F. Mortality after lower extremity fractures in men with spinal cord injury. J. Bone Miner. Res. 2014, 29, 432-439. [CrossRef]

21. Charmetant, C.; Phaner, V.; Condemine, A.; Calmels, P. Diagnosis and treatment of osteoporosis in spinal cord injury patients: A literature review. Ann. Phys. Rehabil. Med. 2010, 53, 655-668. [CrossRef] [PubMed]

22. Morse, L.R.; Giangregorio, L.; Battaglino, R.A.; Holland, R.; Craven, B.C.; Stolzmann, K.L.; Lazzari, A.A.; Sabharwal, S.; Garshick, E. VA-based survey of osteoporosis management in spinal cord injury. PMER 2009, 1, 240-244.

23. Jeremiah, M.P.; Unwin, B.K.; Greenawald, M.H.; Casiano, V.E. Diagnosis and Management of Osteoporosis. Am. Fam. Physician 2015, 92, 261-268.

24. Baker, A.M.; Wagner, D.W.; Kiratli, B.J.; Beaupre, G.S. Pixel-Based DXA-Derived Structural Properties Strongly Correlate with pQCT Measures at the One-Third Distal Femur Site. Ann. Biomed. Eng. 2017, 45, 1247-1254. [CrossRef] [PubMed]

25. Shields, R.K.; Schlechte, J.; Dudley-Javoroski, S.; Zwart, B.D.; Clark, S.D.; Grant, S.A.; Mattiace, V.M. Bone mineral density after spinal cord injury: A reliable method for knee measurement. Arch. Phys. Med. Rehabil. 2005, 86, 1969-1973. [CrossRef] [PubMed]

26. Zheng, X.; Qi, Y.; Zhou, H.; Kang, H.; Tong, Y.; Bi, L. Bone Mineral Density at the Distal Femur and Proximal Tibia and Related Factors During the First Year of Spinal Cord Injury. Int. J. Gen. Med. 2021, 14, 1121-1129. [CrossRef] [PubMed]

27. Cirnigliaro, C.M.; Parrott, J.S.; Myslinski, M.J.; Asselin, P.; Lombard, A.T.; La Fountaine, M.F.; Kirshblum, S.C.; Forrest, G.F.; Dyson-Hudson, T.; Spungen, A.M.; et al. Relationships between T-scores at the hip and bone mineral density at the distal femur and proximal tibia in persons with spinal cord injury. J. Spinal Cord Med. 2020, 43, 685-695. [CrossRef]

28. Shields, R.K.; Dudley-Javoroski, S.; Law, L.A. Electrically induced muscle contractions influence bone density decline after spinal cord injury. Spine 2006, 31, 548-553. [CrossRef]

29. Chen, S.C.; Lai, C.H.; Chan, W.P.; Huang, M.H.; Tsai, H.W.; Chen, J.J. Increases in bone mineral density after functional electrical stimulation cycling exercises in spinal cord injured patients. Disabil. Rehabil. 2005, 27, 1337-1341. [CrossRef] [PubMed]

30. Mohr, T.; Podenphant, J.; Biering-Sorensen, F.; Galbo, H.; Thamsborg, G.; Kjaer, M. Increased bone mineral density after prolonged electrically induced cycle training of paralyzed limbs in spinal cord injured man. Calcif. Tissue Int. 1997, 61, 22-25. [CrossRef]

31. Lai, C.H.; Chang, W.H.; Chan, W.P.; Peng, C.W.; Shen, L.K.; Chen, J.J.; Chen, S.C. Effects of functional electrical stimulation cycling exercise on bone mineral density loss in the early stages of spinal cord injury. J. Rehabil. Med. 2010, 42, 150-154. [CrossRef]

32. Eser, P.; Frotzler, A.; Zehnder, Y.; Denoth, J. Fracture threshold in the femur and tibia of people with spinal cord injury as determined by peripheral quantitative computed tomography. Arch. Phys. Med. Rehabil. 2005, 86, 498-504. [CrossRef] 
33. Edwards, W.B.; Schnitzer, T.J. Bone Imaging and Fracture Risk after Spinal Cord Injury. Curr. Osteoporos. Rep. 2015, 13, 310-317. [CrossRef]

34. Edwards, W.B.; Schnitzer, T.J.; Troy, K.L. The mechanical consequence of actual bone loss and simulated bone recovery in acute spinal cord injury. Bone 2014, 60, 141-147. [CrossRef] [PubMed]

35. Gislason, M.K.; Coupaud, S.; Sasagawa, K.; Tanabe, Y.; Purcell, M.; Allan, D.B.; Tanner, K.E. Prediction of risk of fracture in the tibia due to altered bone mineral density distribution resulting from disuse: A finite element study. Proc. Inst. Mech. Eng. Part $H$ 2014, 228, 165-174. [CrossRef] [PubMed]

36. Edwards, W.B.; Schnitzer, T.J.; Troy, K.L. Reduction in proximal femoral strength in patients with acute spinal cord injury. J. Bone Miner. Res. 2014, 29, 2074-2079. [CrossRef]

37. Dudley-Javoroski, S.; Amelon, R.; Liu, Y.; Saha, P.K.; Shields, R.K. High bone density masks architectural deficiencies in an individual with spinal cord injury. J. Spinal Cord Med. 2014, 37, 349-354. [CrossRef] [PubMed]

38. Lichy, A.M.; Groah, S. Asymmetric lower-limb bone loss after spinal cord injury: Case report. J. Rehabil. Res. Dev. 2012, 49, 221-226. [CrossRef]

39. Yarrow, J.F.; Ye, F.; Balaez, A.; Mantione, J.M.; Otzel, D.M.; Chen, C.; Beggs, L.A.; Baligand, C.; Keener, J.E.; Lim, W.; et al. Bone loss in a new rodent model combining spinal cord injury and cast immobilization. J. Musculoskelet. Neuronal Interact. 2014, 14, 255-266. [PubMed]

40. Edwards, W.B.; Schnitzer, T.J.; Troy, K.L. Bone mineral and stiffness loss at the distal femur and proximal tibia in acute spinal cord injury. Osteoporos. Int. 2014, 25, 1005-1015. [CrossRef] [PubMed]

41. Qin, W.; Bauman, W.A.; Cardozo, C. Bone and muscle loss after spinal cord injury: Organ interactions. Ann. N. Y. Acad. Sci. 2010, 1211, 66-84. [CrossRef]

42. Jiang, S.D.; Jiang, L.S.; Dai, L.Y. Spinal cord injury causes more damage to bone mass, bone structure, biomechanical properties and bone metabolism than sciatic neurectomy in young rats. Osteoporos. Int. 2006, 17, 1552-1561. [CrossRef]

43. Le, B.; Ray, C.; Gonzalez, B.; Miskevics, S.; Weaver, F.M.; Priebe, M.; Carbone, L.D. Laboratory evaluation of secondary causes of bone loss in Veterans with spinal cord injury and disorders. Osteoporos. Int. 2019, 30, 2241-2248. [CrossRef]

44. Yarrow, J.F.; Wnek, R.D.; Conover, C.F.; Reynolds, M.C.; Buckley, K.H.; Kura, J.R.; Sutor, T.W.; Otzel, D.M.; Mattingly, A.J.; Croft, S.; et al. Bone loss after severe spinal cord injury coincides with reduced bone formation and precedes bone blood flow deficits. J. Appl. Physiol. 2021, 131, 1288-1299. [CrossRef] [PubMed]

45. Minaire, P.; Neunier, P.; Edouard, C.; Bernard, J.; Courpron, P.; Bourret, J. Quantitative histological data on disuse osteoporosis: Comparison with biological data. Calcif. Tissue. Res. 1974, 17, 57-73. [CrossRef]

46. Roberts, D.; Lee, W.; Cuneo, R.C.; Wittmann, J.; Ward, G.; Flatman, R.; McWhinney, B.; Hickman, P.E. Longitudinal study of bone turnover after acute spinal cord injury. J. Clin. Endocrinol. Metab. 1998, 83, 415-422. [CrossRef] [PubMed]

47. Maimoun, L.; Couret, I.; Micallef, J.P.; Peruchon, E.; Mariano-Goulart, D.; Rossi, M.; Leroux, J.L.; Ohanna, F. Use of bone biochemical markers with dual-energy X-ray absorptiometry for early determination of bone loss in persons with spinal cord injury. Metabolism 2002, 51, 958-963. [PubMed]

48. Maimoun, L.; Couret, I.; Mariano-Goulart, D.; Dupuy, A.M.; Micallef, J.P.; Peruchon, E.; Ohanna, F.; Cristol, J.P.; Rossi, M.; Leroux, J.L. Changes in osteoprotegerin/RANKL system, bone mineral density, and bone biochemicals markers in patients with recent spinal cord injury. Calcif. Tissue Int. 2005, 76, 404-411. [CrossRef]

49. Reiter, A.L.; Volk, A.; Vollmar, J.; Fromm, B.; Gerner, H.J. Changes of basic bone turnover parameters in short-term and long-term patients with spinal cord injury. Eur. Spine J. 2007, 16, 771-776. [CrossRef]

50. Giangregorio, L.M.; Hicks, A.L.; Webber, C.E.; Phillips, S.M.; Craven, B.C.; Bugaresti, J.M.; McCartney, N. Body weight supported treadmill training in acute spinal cord injury: Impact on muscle and bone. Spinal Cord 2005, 43, 649-657. [CrossRef]

51. Groah, S.L.; Lichy, A.M.; Libin, A.V.; Ljungberg, I. Intensive electrical stimulation attenuates femoral bone loss in acute spinal cord injury. PMER 2010, 2, 1080-1087.

52. Otzel, D.M.; Conover, C.F.; Ye, F.; Harlow, L.M.; Ren, Y.; Wu, Y.; Li, J.; Qin, Y.; Sun, J.; Zheng, S.; et al. Longitudinal Examination of Bone Loss in Male Rats after Moderate-Severe Contusion Spinal Cord Injury. Calcif. Tissue Int. 2019, 104, 79-91. [CrossRef]

53. Beggs, L.A.; Ye, F.; Ghosh, P.; Beck, D.T.; Conover, C.F.; Balaez, A.; Miller, J.R.; Phillips, E.G.; Zheng, N.; Williams, A.A.; et al. Sclerostin inhibition prevents spinal cord injury-induced cancellous bone loss. J. Bone Miner. Res. 2015, 30, 681-689. [CrossRef] [PubMed]

54. Yarrow, J.F.; Conover, C.F.; Beggs, L.A.; Beck, D.T.; Otzel, D.M.; Balaez, A.; Combs, S.M.; Miller, J.R.; Ye, F.; Aguirre, J.I.; et al. Testosterone dose dependently prevents bone and muscle loss in rodents after spinal cord injury. J. Neurotrauma 2014, 31, 834-845. [CrossRef] [PubMed]

55. Morse, L.; Teng, Y.D.; Pham, L.; Newton, K.; Yu, D.; Liao, W.-L.; Kohler, T.; Müller, R.; Graves, D.; Stashenko, P.; et al. Spinal cord injury causes rapid osteoclastic resorption and growth plate abnormalities in growing rats (SCI-induced bone loss in growing rats). Osteoporos. Int. 2008, 19, 645-652. [CrossRef] [PubMed]

56. Liu, D.; Zhao, C.Q.; Li, H.; Jiang, S.D.; Jiang, L.S.; Dai, L.Y. Effects of spinal cord injury and hindlimb immobilization on sublesional and supralesional bones in young growing rats. Bone 2008, 43, 119-125. [CrossRef] [PubMed]

57. Harlow, L.; Sahbani, K.; Nyman, J.S.; Cardozo, C.P.; Bauman, W.A.; Tawfeek, H.A. Daily parathyroid hormone administration enhances bone turnover and preserves bone structure after severe immobilization-induced bone loss. Physiol. Rep. 2017, 5, e13446. [CrossRef] 
58. Yarrow, J.F.; Phillips, E.G.; Conover, C.F.; Bassett, T.E.; Chen, C.; Teurlings, T.; Vasconez, A.; Alerte, J.; Prock, H.; Jiron, J.M.; et al. Testosterone Plus Finasteride Prevents Bone Loss without Prostate Growth in a Rodent Spinal Cord Injury Model. J. Neurotrauma 2017, 34, 2972-2981. [CrossRef]

59. Yarrow, J.F.; Kok, H.J.; Phillips, E.G.; Conover, C.F.; Lee, J.; Bassett, T.E.; Buckley, K.H.; Reynolds, M.C.; Wnek, R.D.; Otzel, D.M.; et al. Locomotor training with adjuvant testosterone preserves cancellous bone and promotes muscle plasticity in male rats after severe spinal cord injury. J. Neurosci. Res. 2020, 98, 843-868. [CrossRef]

60. Qin, L.; Liu, W.; Cao, H.; Xiao, G. Molecular mechanosensors in osteocytes. Bone Res. 2020, 8, 23. [CrossRef]

61. Choi, R.B.; Robling, A.G. The Wnt pathway: An important control mechanism in bone's response to mechanical loading. Bone 2021, 153, 116087. [CrossRef]

62. Qin, W.; Li, X.; Peng, Y.; Harlow, L.M.; Ren, Y.; Wu, Y.; Li, J.; Qin, Y.; Sun, J.; Cardozo, C.C.; et al. Sclerostin antibody preserves the morphology and structure of osteocytes and blocks the severe skeletal deterioration after motor-complete spinal cord injury in rats. J. Bone Miner. Res. 2015, 30, 1994-2004. [CrossRef]

63. Yasuda, H. Discovery of the RANKL/RANK/OPG system. J. Bone Miner. Metab. 2021, 39, 2-11. [CrossRef]

64. Qin, W.; Sun, L.; Cao, J.; Peng, Y.; Collier, L.; Wu, Y.; Creasey, G.; Li, J.; Qin, Y.; Jarvis, J.; et al. The central nervous system (CNS)-independent anti-bone-resorptive activity of muscle contraction and the underlying molecular and cellular signatures. J. Biol. Chem. 2013, 288, 13511-13521. [CrossRef]

65. Cirnigliaro, C.M.; La Fountaine, M.F.; Parrott, J.S.; Kirshblum, S.C.; McKenna, C.; Sauer, S.J.; Shapses, S.A.; Hao, L.; McClure, I.A.; Hobson, J.C.; et al. Administration of Denosumab Preserves Bone Mineral Density at the Knee in Persons with Subacute Spinal Cord Injury: Findings from a Randomized Clinical Trial. JBMR Plus 2020, 4, e10375. [CrossRef]

66. Gifre, L.; Vidal, J.; Carrasco, J.L.; Muxi, A.; Portell, E.; Monegal, A.; Guanabens, N.; Peris, P. Denosumab increases sublesional bone mass in osteoporotic individuals with recent spinal cord injury. Osteoporos. Int. 2016, 27, 405-410. [CrossRef] [PubMed]

67. Gifre, L.; Ruiz-Gaspa, S.; Carrasco, J.L.; Portell, E.; Vidal, J.; Muxi, A.; Monegal, A.; Guanabens, N.; Peris, P. Effect of recent spinal cord injury on the OPG/RANKL system and its relationship with bone loss and the response to denosumab therapy. Osteoporos. Int. 2017, 28, 2707-2715. [CrossRef] [PubMed]

68. Huynh, N.; VonMoss, L.; Smith, D.; Rahman, I.; Felemban, M.F.; Zuo, J.; Rody, W.J., Jr.; McHugh, K.P.; Holliday, L.S. Characterization of Regulatory Extracellular Vesicles from Osteoclasts. J. Dent. Res. 2016, 95, 673-679. [CrossRef]

69. Deng, L.; Wang, Y.; Peng, Y.; Wu, Y.; Ding, Y.; Jiang, Y.; Shen, Z.; Fu, Q. Osteoblast-derived microvesicles: A novel mechanism for communication between osteoblasts and osteoclasts. Bone 2015, 79, 37-42. [CrossRef]

70. Holliday, L.S.; Patel, S.S.; Rody, W.J., Jr. RANKL and RANK in extracellular vesicles: Surprising new players in bone remodeling. Extracell. Vesicles Circ. Nucleic Acids 2021, 2, 18-28. [CrossRef]

71. Ikebuchi, Y.; Aoki, S.; Honma, M.; Hayashi, M.; Sugamori, Y.; Khan, M.; Kariya, Y.; Kato, G.; Tabata, Y.; Penninger, J.; et al. Coupling of bone resorption and formation by RANKL reverse signalling. Nature 2018, 561, 195-200. [CrossRef]

72. Cappariello, A.; Loftus, A.; Muraca, M.; Maurizi, A.; Rucci, N.; Teti, A. Osteoblast-Derived Extracellular Vesicles Are Biological Tools for the Delivery of Active Molecules to Bone. J. Bone Miner. Res. 2018, 33, 517-533. [CrossRef]

73. Lin, C.; Jiang, X.; Dai, Z.; Guo, X.; Weng, T.; Wang, J.; Li, Y.; Feng, G.; Gao, X.; He, L. Sclerostin mediates bone response to mechanical unloading through antagonizing Wnt/beta-catenin signaling. J. Bone Miner. Res. 2009, 24, 1651-1661. [CrossRef]

74. Tu, X.; Rhee, Y.; Condon, K.W.; Bivi, N.; Allen, M.R.; Dwyer, D.; Stolina, M.; Turner, C.H.; Robling, A.G.; Plotkin, L.I.; et al. Sost downregulation and local Wnt signaling are required for the osteogenic response to mechanical loading. Bone 2012, 50, $209-217$. [CrossRef]

75. Metzger, C.E.; Gong, S.; Aceves, M.; Bloomfield, S.A.; Hook, M.A. Osteocytes reflect a pro-inflammatory state following spinal cord injury in a rodent model. Bone 2019, 120, 465-475. [CrossRef]

76. Qin, W.; Zhao, W.; Li, X.; Peng, Y.; Harlow, L.M.; Li, J.; Qin, Y.; Pan, J.; Wu, Y.; Ran, L.; et al. Mice with sclerostin gene deletion are resistant to the severe sublesional bone loss induced by spinal cord injury. Osteoporos. Int. 2016, 27, 3627-3636. [CrossRef]

77. Zhao, W.; Li, X.; Peng, Y.; Qin, Y.; Pan, J.; Li, J.; Xu, A.; Ominsky, M.S.; Cardozo, C.; Feng, J.Q.; et al. Sclerostin Antibody Reverses the Severe Sublesional Bone Loss in Rats after Chronic Spinal Cord Injury. Calcif. Tissue Int. 2018, 103, 443-454. [CrossRef] [PubMed]

78. Otzel, D.M.; Lee, J.; Ye, F.; Borst, S.E.; Yarrow, J.F. Activity-Based Physical Rehabilitation with Adjuvant Testosterone to Promote Neuromuscular Recovery after Spinal Cord Injury. Int. J. Mol. Sci. 2018, 19, 1701. [CrossRef]

79. Harkema, S.J.; Hillyer, J.; Schmidt-Read, M.; Ardolino, E.; Sisto, S.A.; Behrman, A.L. Locomotor training: As a treatment of spinal cord injury and in the progression of neurologic rehabilitation. Arch. Phys. Med. Rehabil. 2012, 93, 1588-1597. [CrossRef]

80. Mehrholz, J.; Kugler, J.; Pohl, M. Locomotor training for walking after spinal cord injury. Cochrane Database Syst. Rev. 2012, 11, CD006676. [CrossRef] [PubMed]

81. Nam, K.Y.; Kim, H.J.; Kwon, B.S.; Park, J.W.; Lee, H.J.; Yoo, A. Robot-assisted gait training (Lokomat) improves walking function and activity in people with spinal cord injury: A systematic review. J. Neuroeng. Rehabil. 2017, 14, 24. [CrossRef] [PubMed]

82. Wessels, M.; Lucas, C.; Eriks, I.; de Groot, S. Body weight-supported gait training for restoration of walking in people with an incomplete spinal cord injury: A systematic review. J. Rehabil. Med. 2010, 42, 513-519. [CrossRef]

83. Knikou, M. Neurophysiological characteristics of human leg muscle action potentials evoked by transcutaneous magnetic stimulation of the spine. Bioelectromagnetics 2013, 34, 200-210. [CrossRef] 
84. Smith, A.C.; Knikou, M. A Review on Locomotor Training after Spinal Cord Injury: Reorganization of Spinal Neuronal Circuits and Recovery of Motor Function. Neural Plast. 2016, 2016, 1216258. [CrossRef]

85. Knikou, M.; Mummidisetty, C.K. Locomotor training improves premotoneuronal control after chronic spinal cord injury. J. Neurophysiol. 2014, 111, 2264-2275. [CrossRef]

86. Smith, A.C.; Mummidisetty, C.K.; Rymer, W.Z.; Knikou, M. Locomotor training alters the behavior of flexor reflexes during walking in human spinal cord injury. J. Neurophysiol. 2014, 112, 2164-2175. [CrossRef]

87. Thomas, S.L.; Gorassini, M.A. Increases in corticospinal tract function by treadmill training after incomplete spinal cord injury. J. Neurophysiol. 2005, 94, 2844-2855. [CrossRef] [PubMed]

88. Krishnan, V.; Kindig, M.; Mirbagheri, M. Robotic-assisted locomotor training enhances ankle performance in adults with incomplete spinal cord injury. J. Rehabil. Med. 2016, 48, 781-786. [CrossRef] [PubMed]

89. Blum, R.; Konnerth, A. Neurotrophin-mediated rapid signaling in the central nervous system: Mechanisms and functions. Physiology 2005, 20, 70-78. [CrossRef] [PubMed]

90. Kohrt, W.M.; Bloomfield, S.A.; Little, K.D.; Nelson, M.E.; Yingling, V.R. American College of Sports Medicine Position Stand: Physical activity and bone health. Med. Sci. Sports Exerc. 2004, 36, 1985-1996. [CrossRef] [PubMed]

91. Orwoll, E.S.; Adler, R.A.; Amin, S.; Binkley, N.; Lewiecki, E.M.; Petak, S.M.; Shapses, S.A.; Sinaki, M.; Watts, N.B.; Sibonga, J.D. Skeletal health in long-duration astronauts: Nature, assessment, and management recommendations from the NASA Bone Summit. J. Bone Miner. Res. 2013, 28, 1243-1255. [CrossRef]

92. Robling, A.G.; Turner, C.H. Mechanical signaling for bone modeling and remodeling. Crit. Rev. Eukaryot. Gene Expr. 2009, 19, 319-338. [CrossRef]

93. Weaver, F.M.; Etingen, B.; Guihan, M.; Ray, C.; Priebe, M.; Burns, S.; Carbone, L. Spinal cord injury providers' perspectives on managing sublesional osteoporosis. J. Spinal Cord Med. 2020, 43, 428-434. [CrossRef]

94. Soleyman-Jahi, S.; Yousefian, A.; Maheronnaghsh, R.; Shokraneh, F.; Zadegan, S.A.; Soltani, A.; Hosseini, S.M.; Vaccaro, A.R.; Rahimi-Movaghar, V. Evidence-based prevention and treatment of osteoporosis after spinal cord injury: A systematic review. Eur. Spine J. 2018, 27, 1798-1814. [CrossRef]

95. Panisset, M.G.; Galea, M.P.; El-Ansary, D. Does early exercise attenuate muscle atrophy or bone loss after spinal cord injury? Spinal Cord 2016, 54, 84-92. [CrossRef]

96. Bloomfield, S.A.; Mysiw, W.J.; Jackson, R.D. Bone mass and endocrine adaptations to training in spinal cord injured individuals. Bone 1996, 19, 61-68. [CrossRef]

97. Mobarake, B.G.; Banitalebi, E.; Ebrahimi, A.; Ghafari, M. Effects of progressive locomotor treadmill compared to conventional training on bone mineral density and bone remodeling in paraplegia. Middle East J. Rehabil. Health Stud. 2017, 4, e37732.

98. Craven, B.C.; Giangregorio, L.M.; Alavinia, S.M.; Blencowe, L.A.; Desai, N.; Hitzig, S.L.; Masani, K.; Popovic, M.R. Evaluating the efficacy of functional electrical stimulation therapy assisted walking after chronic motor incomplete spinal cord injury: Effects on bone biomarkers and bone strength. J. Spinal Cord Med. 2017, 40, 748-758. [CrossRef] [PubMed]

99. Arija-Blazquez, A.; Ceruelo-Abajo, S.; Diaz-Merino, M.S.; Godino-Duran, J.A.; Martinez-Dhier, L.; Martin, J.L.; Florensa-Vila, J. Effects of electromyostimulation on muscle and bone in men with acute traumatic spinal cord injury: A randomized clinical trial. J. Spinal Cord Med. 2014, 37, 299-309. [CrossRef]

100. Giangregorio, L.M.; Webber, C.E.; Phillips, S.M.; Hicks, A.L.; Craven, B.C.; Bugaresti, J.M.; McCartney, N. Can body weight supported treadmill training increase bone mass and reverse muscle atrophy in individuals with chronic incomplete spinal cord injury? Appl. Physiol. Nutr. Metab. 2006, 31, 283-291. [CrossRef] [PubMed]

101. Edwards, W.B.; Simonian, N.; Haider, I.T.; Anschel, A.S.; Chen, D.; Gordon, K.E.; Gregory, E.K.; Kim, K.H.; Parachuri, R.; Troy, K.L.; et al. Effects of Teriparatide and Vibration on Bone Mass and Bone Strength in People with Bone Loss and Spinal Cord Injury: A Randomized, Controlled Trial. J. Bone Miner. Res. 2018, 33, 1729-1740. [CrossRef] [PubMed]

102. Wuermser, L.A.; Beck, L.A.; Lamb, J.L.; Atkinson, E.J.; Amin, S. The effect of low-magnitude whole body vibration on bone density and microstructure in men and women with chronic motor complete paraplegia. J. Spinal Cord Med. 2015, 38, 178-186. [CrossRef]

103. Astorino, T.A.; Harness, E.T.; Witzke, K.A. Effect of chronic activity-based therapy on bone mineral density and bone turnover in persons with spinal cord injury. Eur. J. Appl. Physiol. 2013, 113, 3027-3037. [CrossRef]

104. Zhao, W.; Peng, Y.; Hu, Y.; Guo, X.E.; Li, J.; Cao, J.; Pan, J.; Feng, J.Q.; Cardozo, C.; Jarvis, J.; et al. Electrical stimulation of hindlimb skeletal muscle has beneficial effects on sublesional bone in a rat model of spinal cord injury. Bone 2021, 144, 115825. [CrossRef]

105. Zamarioli, A.; Battaglino, R.A.; Morse, L.R.; Sudhakar, S.; Maranho, D.A.; Okubo, R.; Volpon, J.B.; Shimano, A.C. Standing frame and electrical stimulation therapies partially preserve bone strength in a rodent model of acute spinal cord injury. Am. J. Phys. Med. Rehabil. 2013, 92, 402-410. [CrossRef] [PubMed]

106. Lambach, R.L.; Stafford, N.E.; Kolesar, J.A.; Kiratli, B.J.; Creasey, G.H.; Gibbons, R.S.; Andrews, B.J.; Beaupre, G.S. Bone changes in the lower limbs from participation in an FES rowing exercise program implemented within two years after traumatic spinal cord injury. J. Spinal Cord Med. 2020, 43, 306-314. [CrossRef] [PubMed]

107. Goemaere, S.; Van Laere, M.; De Neve, P.; Kaufman, J.M. Bone mineral status in paraplegic patients who do or do not perform standing. Osteoporos. Int. 1994, 4, 138-143. [CrossRef] [PubMed] 
108. Rodgers, M.M.; Glaser, R.M.; Figoni, S.F.; Hooker, S.P.; Ezenwa, B.N.; Collins, S.R.; Mathews, T.; Suryaprasad, A.G.; Gupta, S.C. Musculoskeletal responses of spinal cord injured individuals to functional neuromuscular stimulation-induced knee extension exercise training. J. Rehabil. Res. Dev. 1991, 28, 19-26. [CrossRef] [PubMed]

109. Alekna, V.; Tamulaitiene, M.; Sinevicius, T.; Juocevicius, A. Effect of weight-bearing activities on bone mineral density in spinal cord injured patients during the period of the first two years. Spinal Cord 2008, 46, 727-732. [CrossRef]

110. Ben, M.; Harvey, L.; Denis, S.; Glinsky, J.; Goehl, G.; Chee, S.; Herbert, R.D. Does 12 weeks of regular standing prevent loss of ankle mobility and bone mineral density in people with recent spinal cord injuries? Aust. J. Physiother. 2005, 51, 251-256. [CrossRef]

111. Clark, J.M.; Jelbart, M.; Rischbieth, H.; Strayer, J.; Chatterton, B.; Schultz, C.; Marshall, R. Physiological effects of lower extremity functional electrical stimulation in early spinal cord injury: Lack of efficacy to prevent bone loss. Spinal Cord 2007, 45, 78-85. [CrossRef]

112. De Bruin, E.D.; Frey-Rindova, P.; Herzog, R.E.; Dietz, V.; Dambacher, M.A.; Stussi, E. Changes of tibia bone properties after spinal cord injury: Effects of early intervention. Arch. Phys. Med. Rehabil. 1999, 80, 214-220. [CrossRef]

113. Dudley-Javoroski, S.; Saha, P.K.; Liang, G.; Li, C.; Gao, Z.; Shields, R.K. High dose compressive loads attenuate bone mineral loss in humans with spinal cord injury. Osteoporos. Int. 2012, 23, 2335-2346. [CrossRef]

114. Frey-Rindova, P.; de Bruin, E.D.; Stussi, E.; Dambacher, M.A.; Dietz, V. Bone mineral density in upper and lower extremities during 12 months after spinal cord injury measured by peripheral quantitative computed tomography. Spinal Cord 2000, 38, 26-32. [CrossRef]

115. Shields, R.K.; Dudley-Javoroski, S. Musculoskeletal plasticity after acute spinal cord injury: Effects of long-term neuromuscular electrical stimulation training. J. Neurophysiol. 2006, 95, 2380-2390. [CrossRef] [PubMed]

116. Dudley-Javoroski, S.; Shields, R.K. Active-resisted stance modulates regional bone mineral density in humans with spinal cord injury. J. Spinal Cord Med. 2013, 36, 191-199. [CrossRef] [PubMed]

117. Davis, R.; Sanborn, C.; Nichols, D.; Bazett-Jones, D.M.; Dugan, E.L. The Effects of Whole Body Vibration on Bone Mineral Density for a Person with a Spinal Cord Injury: A Case Study. Adapt. Phys. Act. Q. 2010, 27, 60-72. [CrossRef] [PubMed]

118. Ogilvie, C.; Bowker, P.; Rowley, D.I. The physiological benefits of paraplegic orthotically aided walking. Paraplegia 1993, 31, 111-115. [CrossRef]

119. Forrest, G.F.; Sisto, S.A.; Barbeau, H.; Kirshblum, S.C.; Wilen, J.; Bond, Q.; Bentson, S.; Asselin, P.; Cirnigliaro, C.M.; Harkema, S. Neuromotor and musculoskeletal responses to locomotor training for an individual with chronic motor complete AIS-B spinal cord injury. J. Spinal Cord Med. 2008, 31, 509-521. [CrossRef]

120. Beck, L.; Veith, D.; Linde, M.; Gill, M.; Calvert, J.; Grahn, P.; Garlanger, K.; Husmann, D.; Lavrov, I.; Sayenko, D.; et al. Impact of long-term epidural electrical stimulation enabled task-specific training on secondary conditions of chronic paraplegia in two humans. J. Spinal Cord Med. 2020, 44, 800-805. [CrossRef]

121. Coupaud, S.; Jack, L.P.; Hunt, K.J.; Allan, D.B. Muscle and bone adaptations after treadmill training in incomplete Spinal Cord Injury: A case study using peripheral Quantitative Computed Tomography. J. Musculoskelet. Neuronal Interact. $2009,9,288-297$.

122. Dolbow, D.R.; Gorgey, A.S.; Khalil, R.K.; Gater, D.R. Effects of a fifty-six month electrical stimulation cycling program after tetraplegia: Case report. J. Spinal Cord Med. 2017, 40, 485-488. [CrossRef]

123. Dolbow, D.R.; Gorgey, A.S.; Gater, D.R.; Moore, J.R. Body composition changes after 12 months of FES cycling: Case report of a 60-year-old female with paraplegia. Spinal Cord 2014, 52 (Suppl. S1), S3-S4. [CrossRef]

124. Gibbons, R.S.; McCarthy, I.D.; Gall, A.; Stock, C.G.; Shippen, J.; Andrews, B.J. Can FES-rowing mediate bone mineral density in SCI: A pilot study. Spinal Cord 2014, 52 (Suppl. S3), S4-S5. [CrossRef]

125. Deley, G.; Denuziller, J.; Casillas, J.M.; Babault, N. One year of training with FES has impressive beneficial effects in a 36-year-old woman with spinal cord injury. J. Spinal Cord Med. 2017, 40, 107-112. [CrossRef] [PubMed]

126. Pacy, P.J.; Hesp, R.; Halliday, D.A.; Katz, D.; Cameron, G.; Reeve, J. Muscle and bone in paraplegic patients, and the effect of functional electrical stimulation. Clin. Sci. 1988, 75, 481-487. [CrossRef] [PubMed]

127. Goktepe, A.S.; Tugcu, I.; Yilmaz, B.; Alaca, R.; Gunduz, S. Does standing protect bone density in patients with chronic spinal cord injury? J. Spinal Cord Med. 2008, 31, 197-201. [CrossRef] [PubMed]

128. Kunkel, C.F.; Scremin, A.M.; Eisenberg, B.; Garcia, J.F.; Roberts, S.; Martinez, S. Effect of "standing" on spasticity, contracture, and osteoporosis in paralyzed males. Arch. Phys. Med. Rehabil. 1993, 74, 73-78.

129. Karelis, A.D.; Carvalho, L.P.; Castillo, M.J.; Gagnon, D.H.; Aubertin-Leheudre, M. Effect on body composition and bone mineral density of walking with a robotic exoskeleton in adults with chronic spinal cord injury. J. Rehabil. Med. 2017, 49, 84-87. [CrossRef]

130. Thoumie, P.; Le Claire, G.; Beillot, J.; Dassonville, J.; Chevalier, T.; Perrouin-Verbe, B.; Bedoiseau, M.; Busnel, M.; Cormerais, A.; Courtillon, A.; et al. Restoration of functional gait in paraplegic patients with the RGO-II hybrid orthosis. A multicenter controlled study. II: Physiological evaluation. Paraplegia 1995, 33, 654-659. [CrossRef]

131. Needham-Shropshire, B.M.; Broton, J.G.; Klose, K.J.; Lebwohl, N.; Guest, R.S.; Jacobs, P.L. Evaluation of a training program for persons with SCI paraplegia using the Parastep 1 ambulation system: Part 3. Lack of effect on bone mineral density. Arch. Phys. Med. Rehabil. 1997, 78, 799-803. [CrossRef]

132. Griffin, L.; Decker, M.J.; Hwang, J.Y.; Wang, B.; Kitchen, K.; Ding, Z.; Ivy, J.L. Functional electrical stimulation cycling improves body composition, metabolic and neural factors in persons with spinal cord injury. J. Electromyogr. Kinesiol. 2009, 19, 614-622. [CrossRef] 
133. BeDell, K.K.; Scremin, A.M.; Perell, K.L.; Kunkel, C.F. Effects of functional electrical stimulation-induced lower extremity cycling on bone density of spinal cord-injured patients. Am. J. Phys. Med. Rehabil. 1996, 75, 29-34. [CrossRef]

134. Frotzler, A.; Coupaud, S.; Perret, C.; Kakebeeke, T.H.; Hunt, K.J.; Donaldson, N.N.; Eser, P. High-volume FES-cycling partially reverses bone loss in people with chronic spinal cord injury. Bone 2008, 43, 169-176. [CrossRef] [PubMed]

135. Leeds, E.M.; Klose, K.J.; Ganz, W.; Serafini, A.; Green, B.A. Bone mineral density after bicycle ergometry training. Arch. Phys. Med. Rehabil. 1990, 71, 207-209.

136. Dudley-Javoroski, S.; Petrie, M.A.; McHenry, C.L.; Amelon, R.E.; Saha, P.K.; Shields, R.K. Bone architecture adaptations after spinal cord injury: Impact of long-term vibration of a constrained lower limb. Osteoporos. Int. 2016, 27, 1149-1160. [CrossRef] [PubMed]

137. Belanger, M.; Stein, R.B.; Wheeler, G.D.; Gordon, T.; Leduc, B. Electrical stimulation: Can it increase muscle strength and reverse osteopenia in spinal cord injured individuals? Arch. Phys. Med. Rehabil. 2000, 81, 1090-1098. [CrossRef] [PubMed]

138. Shields, R.K.; Dudley-Javoroski, S. Musculoskeletal adaptations in chronic spinal cord injury: Effects of long-term soleus electrical stimulation training. Neurorehabil. Neural. Repair. 2007, 21, 169-179. [CrossRef] [PubMed]

139. Johnston, T.E.; Marino, R.J.; Oleson, C.V.; Schmidt-Read, M.; Leiby, B.E.; Sendecki, J.; Singh, H.; Modlesky, C.M. Musculoskeletal Effects of 2 Functional Electrical Stimulation Cycling Paradigms Conducted at Different Cadences for People with Spinal Cord Injury: A Pilot Study. Arch. Phys. Med. Rehabil. 2016, 97, 1413-1422. [CrossRef]

140. Hangartner, T.N.; Rodgers, M.M.; Glaser, R.M.; Barre, P.S. Tibial bone density loss in spinal cord injured patients: Effects of FES exercise. J. Rehabil. Res. Dev. 1994, 31, 50-61.

141. Morse, L.R.; Troy, K.L.; Fang, Y.; Nguyen, N.; Battaglino, R.; Goldstein, R.F.; Gupta, R.; Taylor, J.A. Combination Therapy with Zoledronic Acid and FES-Row Training Mitigates Bone Loss in Paralyzed Legs: Results of a Randomized Comparative Clinical Trial. JBMR Plus 2019, 3, e10167. [CrossRef]

142. Draghici, A.E.; Taylor, J.A.; Bouxsein, M.L.; Shefelbine, S.J. Effects of FES-Rowing Exercise on the Time-Dependent Changes in Bone Microarchitecture after Spinal Cord Injury: A Cross-Sectional Investigation. JBMR Plus 2019, 3, e10200. [CrossRef]

143. Eser, P.C.; Donaldson Nde, N.; Knecht, H.; Stussi, E. Influence of different stimulation frequencies on power output and fatigue during FES-cycling in recently injured SCI people. IEEE Trans. Neural. Syst. Rehabil. Eng. 2003, 11, 236-240. [CrossRef]

144. Fang, Y.; Morse, L.R.; Nguyen, N.; Battaglino, R.A.; Goldstein, R.F.; Troy, K.L. Functional electrical stimulation (FES)-assisted rowing combined with zoledronic acid, but not alone, preserves distal femur strength and stiffness in people with chronic spinal cord injury. Osteoporos. Int. 2021, 32, 549-558. [CrossRef] [PubMed]

145. Tamaki, H.; Yotani, K.; Ogita, F.; Hayao, K.; Nakagawa, K.; Sugawara, K.; Kirimoto, H.; Onishi, H.; Kasuga, N.; Yamamoto, N. Electrical Stimulation of Denervated Rat Skeletal Muscle Ameliorates Bone Fragility and Muscle Loss in Early-Stage Disuse Musculoskeletal Atrophy. Calcif. Tissue Int. 2017, 100, 420-430. [CrossRef] [PubMed]

146. Midura, R.J.; Dillman, C.J.; Grabiner, M.D. Low amplitude, high frequency strains imposed by electrically stimulated skeletal muscle retards the development of osteopenia in the tibiae of hindlimb suspended rats. Med. Eng. Phys. 2005, 27, 285-293. [CrossRef] [PubMed]

147. Wu, Y.; Collier, L.; Qin, W.; Creasey, G.; Bauman, W.A.; Jarvis, J.; Cardozo, C. Electrical stimulation modulates Wnt signaling and regulates genes for the motor endplate and calcium binding in muscle of rats with spinal cord transection. BMC Neurosci. 2013, 14, 81. [CrossRef]

148. Holman, M.E.; Chang, G.; Ghatas, M.P.; Saha, P.K.; Zhang, X.; Khan, M.R.; Sima, A.P.; Adler, R.A.; Gorgey, A.S. Bone and non-contractile soft tissue changes following open kinetic chain resistance training and testosterone treatment in spinal cord injury: An exploratory study. Osteoporos. Int. 2021, 32, 1321-1332. [CrossRef]

149. Chandran, V.D.; Lambach, R.L.; Gibbons, R.S.; Andrews, B.J.; Beaupre, G.S.; Pal, S. Tibiofemoral forces during FES rowing in individuals with spinal cord injury. Comput. Methods Biomech. Biomed. Eng. 2020, 24, 231-244. [CrossRef] 